Rectifiability and Harmonic Measure

\title{
A Dissertation
}

presented to

the Faculty of the Graduate School

University of Missouri

In Partial Fulfillment

of the Requirements for the Degree

Doctor of Philosophy

by

Simon Bortz

Dr. Steven Hofmann, Dissertation Supervisor

May 2016 
The undersigned, appointed by the Dean of the Graduate School, have examined the dissertation entitled

\section{Rectifiability and Harmonic Measure}

presented by Simon Bortz, a candidate for the degree of Doctor of Philosophy of Mathematics, and hereby certify that in their opinion it is worthy of acceptance.

Professor Steven Hofmann

Professor Marius Mitrea

Professor Steven Neal

Professor Igor Verbitsky 


\section{ACKNOWLEDGEMENTS}

First, I would like to thank my advisor Steve Hofmann. His patience was infinite and his guidance was invaluable. For the confidence he instilled in me, and the many opportunities he has provided, I will be eternally grateful.

I thank my wife, Tiffanie, for making sacrifices to enable me to pursue this endeavour and her bottomless faith in my ability to succeed.

I am also grateful to José Maria (Chema) Martell for his contributions and advice in my thesis. His work with Steve Hofmann and Svitlana Mayboroda is the basis for the second chapter.

I also thank Marius Mitrea, Steven Neal, and Igor Verbitsky; for serving as members of my committee. Igor Verbitsky has also stood as an excellent mentor and teacher to me.

Finally, I thank all of the faculty who have taught courses I have taken. I have learned something important from every experience.

All of the work contained in my thesis with exception of Theorem 2.4.9, is joint work with Steve Hofmann. Chapter 3 consists of joint work with Murat Akman, Steve Hofmann and Chema Martell. Theorem 2.4.9 is joint work with Chema Martell. The author was supported by NSF grant DMS-1361701. 


\section{Contents}

Acknowledgements $\quad$ ii

Abstract $\quad$ v

1 Introduction 1

1.1 Notation . . . . . . . . . . . . . . . . . . 6

2 Uniform Rectifiability and Harmonic Measure 9

2.1 Notation and Definitions . . . . . . . . . . . . . . . . 9

2.2 A bilateral corona decomposition and corona type approximation by chord arc domains: Preliminaries for Theorem 2.3.1 . . . . . . . . . 16

2.3 Carleson measures and Theorem 2.3.1 . . . . . . . . . . . 25

2.4 Theorems 2.4.1 and 2.4.9: Applications to Harmonic Measure . . . . 35

3 Rectifiability and Harmonic Measure $\quad 44$

3.1 Notation and Definitions . . . . . . . . . . . . . 45

3.2 Cone sets, harmonic measure and rectifiability . . . . . . . . . . . 48

3.3 Counterexamples . . . . . . . . . . . . . . . . 59

4 A Two Phase Free Boundary Problem 65

4.1 Notation and Definitions . . . . . . . . . . . . . 65 
4.2 Preliminary Estimates and Observations . . . . . . . . . . . . 68

4.3 Theorems 4.3 .1 and $4.3 .18 \ldots \ldots$. . . . . . . . . . . . . . 73

$\begin{array}{lr}\text { Bibliography } & 83\end{array}$

Vita 


\title{
Rectifiability and Harmonic Measure
}

\author{
Simon Bortz \\ Dr. Steven Hofmann, Dissertation Supervisor
}

\begin{abstract}
This dissertation is concerned with the interplay of the geometry of the boundary of a given domain (or open set) and its harmonic measure. First, we prove structure theorems for uniformly rectifiable sets. These structure theorems yield information about harmonic measure. Then we turn our attention to sets that are merely rectifiable. We show that under some mild hypotheses, if the boundary of a domain is rectifiable then surface measure is absolutely continuous with respect to harmonic measure. Finally, we explore the other direction of the connection between geometry of the boundary and harmonic measure. We show that (under certain background hypotheses) weak regularity of the Poisson kernel on both sides of a domain yields the same regularity for the unit outer normal to the domain.
\end{abstract}




\section{Chapter 1}

\section{Introduction}

After the introduction this dissertation is divided into three chapters. The Chapters 2 and 3 are concerned with (qualitative and quantitative) absolute continuity of surface measure with respect to harmonic measure and absolute continuity of harmonic measure with respect to surface measure in a certain class of domains. The fourth chapter is concerned with free boundary problems, in which one hopes to use the existence of a "nice" solution to a PDE in order to gain information about the boundary.

The work concerning absolute continuity is part of a general program to prove qualitative and quantitative analogues of the classical F. and M. Riesz Theorem [RR] in higher dimensions. The F. and M. Riesz Theorem states that for a simply connected domain in the complex plane with a rectifiable boundary, harmonic measure and arclength measure are mutually absolutely continuous. A quantitative version of this theorem (again in the plane) was obtained by Lavrentiev [La]. It turns out that some strong connectivity hypothesis is necessary to infer that harmonic measure is absolutely continuous with respect to surface measure. Indeed, Bishop and Jones $[\mathrm{BiJ}]$ have presented a counter-example to show that the result of $[\mathrm{RR}]$ may fail in the absence of sufficient connectivity. In [BiJ], the authors construct a connected open set which is the complement of a (uniformly) 1-rectifiable set for which harmonic 
measure fails to be absolutely continuous with respect to surface measure. Thus, roughly speaking, rectifiability plus strong connectivity implies absolute continuity of harmonic measure with respect to surface measure, but rectifiability alone does not. In higher dimensions, quantitative (scale-invariant) versions of the F. and M. Riesz Theorem were obtained in special settings. Dahlberg [Da] showed that in a Lipschitz domain, harmonic measure is in the reverse Hölder class with exponent 2; this implies that harmonic measure is in the Muckenhoupt class $A_{\infty}$ (with respect to surface measure), which is scale invariant quantitative version of mutual absolute continuity. Later, it was shown by David and Jerison [DJ], and independently, by Semmes [Se], that in NTA domains with ADR (Ahlfors-David Regular) boundaries (all terminology and notation to be defined below), again harmonic measure belongs to the Muckenhoupt class $A_{\infty}$. David and Jerison accomplished this by showing that NTA domains with ADR boundaries contain "big pieces of interior Lipschitz subdomains". On the other hand, we know that the analogue of $[\mathrm{RR}]$ fails to hold in higher dimensions. $\mathrm{Wu}[\mathrm{W}]$ and Ziemer $[\mathrm{Z}]$ produced examples of topological 2-spheres in $\mathbb{R}^{3}$ with locally finite perimeter in which harmonic measure is fails to be absolutely continuous with respect to surface measure and surface measure fails to be absolutely continuous with respect to harmonic measure respectively.

As noted above, the counter-example of [BiJ] shows that in the setting of domains with (uniformly) rectifiable boundary that one cannot expect to have harmonic measure quantitatively (or even qualitatively) absolutely continuous with respect to surface measure. Nonetheless, the author (in joint work with Steve Hofmann) obtained a structure theorem (Theorem 2.3.1) for uniformly rectifiable sets of co-dimension 1, 
which yields in particular that the F. and M. Riesz theorem holds for every such set $E$ (viewed as the boundary of an open set $\Omega=\mathbb{R}^{n+1} \backslash E$ ), in a "big pieces" sense. On the other hand in [HM3], the "full" converse holds, that is, if $E \subset \mathbb{R}^{n+1}$ is a closed ADR set and harmonic measure for $\mathbb{R}^{n+1} \backslash E$ is in the weak- $A_{\infty}$ class with respect to surface measure then $E$ is uniformly rectifiable. Combining these results we are able to characterize uniformly rectifiable sets of co-dimension 1 (see Theorem 2.4.1).

In the other direction, that is, showing surface measure is absolutely continuous with respect to harmonic measure, the hypothesis of [DJ] can be relaxed to obtain (qualitative) absolute continuity. In [Ba] (see also [Azz]), the author proved that surface measure $\sigma$ is absolutely continuous with respect to $\omega$ in an NTA domain $\Omega$ with locally finite perimeter, thus replacing the upper Ahlfors-David regularity ("ADR") condition on $\partial \Omega$ assumed in $[\mathrm{DJ}]$ by a weaker qualitative condition, namely, local finiteness of $\sigma$ (the lower ADR bound holds automatically for NTA domains, by the local isoperimetric inequality). A refinement of the result in [Ba] was obtained in $[\mathrm{Mo}]$, where it is shown that for a uniform domain of locally finite perimeter, with rectifiable boundary satisfying the lower ADR condition, surface measure is again absolutely continuous with respect to harmonic measure. Independently, [ABaHM] obtained this result (as well as its converse) assuming "full" (i.e., upper and lower) ADR.

Let us note that in all of the aforementioned results (which give surface measure is absolutely continuous with respect to harmonic measure) there is a strong connectivity hypothesis, that is, in $[R R]$ simple connectivity is assumed or in [Da], [DJ], [Ba], [Mo] and $[\mathrm{ABaHM}]$ the Harnack chain condition is assumed (or implied). We show that one 
can modify the proof of Theorem 2.3.1 to show that if $\Omega$ is a domain with uniformly rectifiable boundary $\partial \Omega$ and $\Omega$ satisfies a interior corkscrew condition then surface measure is absolutely continuous with respect to harmonic measure. In Theorem 3.2.31, we are able to weaken these hypotheses considerable to only requiring the domain $\Omega$ to have rectifiable boundary, $\partial \Omega$, which satisfies a weak openness condition and whose surface measure is locally finite and satisfies a weak lower ADR condition. The technique here is much like that in McMillian's Theorem [GM, pp 207-210] which states that in the plane the restrictions of harmonic measure and surface measure to the cone set are mutually absolutely continuous. In fact, we will first show that if $K$ is the cone set for a domain then $\sigma\llcorner K \ll \omega L K$; Theorem 3.2.31 will follow quickly from this as soon as we show the cone set has full surface measure.

Let us note that several of the results in this dissertation dispense with connectivity entirely, that is, we will treat, $E$, a set of co-dimension 1 in $\mathbb{R}^{n+1}$ as the boundary of the open set $\mathbb{R}^{n+1} \backslash E$. In this setting we must be careful, if $\mathbb{R}^{n+1} \backslash E$ has more than one component then the null sets of harmonic measure with pole at $X, \omega^{X}$, will change for different $X$. (In general, the Harnack inequality gives that if $\omega^{X}$ is the harmonic measure for $\mathbb{R}^{n+1} \backslash E$ with pole at $X$, then $\omega^{X_{1}}$ and $\omega^{X_{2}}$ have the same null sets provided that $X_{1}$ and $X_{2}$ belong to the same connected component.) In this setting, it seems to be appropriate to establish a non-degeneracy condition for harmonic measure in lieu of absolute continuity (of surface measure with respect to harmonic measure), that is, we hope to be able to say if $F \subset E$ with positive surface measure then $\omega^{X}(F)>0$ for some $X \in \mathbb{R}^{n+1} \backslash E$. This is what we establish in Theorem 3.2.28. 
In the fourth chapter of this dissertation we study a two phase free boundary problem for the Poisson kernel. The study of free boundary problems for Poisson kernels, in which the regularity of the Poisson kernel $k$ associated to a domain $\Omega$, is shown to imply regularity of its boundary $\partial \Omega$, began with the work of Alt and Caffarelli $[\mathrm{AC}]$. In $[\mathrm{AC}]$ it is shown that in the presence of sufficient Reifenberg flatness, plus Ahlfors-David regularity of the boundary, $\log k \in C^{\alpha} \Longrightarrow \nu \in C^{\beta}$, where $\nu$ is the unit normal to $\partial \Omega$. In [J], Jerison showed that $\beta$ can be taken to be equal to $\alpha$. In the same work, Jerison studied the regularity of the unit normal under the assumption that $\log k$ was continuous and that $\partial \Omega$ was Lipschitz. The converse to the Alt-Caffarelli-Jerison $C^{\alpha}$ result is a classical theorem of Kellog [Kel].

Kenig and Toro investigated what may be considered the 'end point' case of the results in $[\mathrm{AC}]$, again under the assumption of Reifenberg flatness and Ahlfors-David regularity. In [KT1] and [KT3], they showed that given those background hypotheses, one obtains

$$
\log k \in V M O(d \sigma) \Longleftrightarrow \nu \in V M O(d \sigma)
$$

(more precisely, for the direction " $\Longrightarrow$ ", they show that $\nu \in V M O_{l o c}(d \sigma)$; we shall return to this point later), where for a domain $\Omega \subset \mathbb{R}^{n+1}$, we let $\sigma:=H^{n} \mathrm{~L} \partial \Omega$ denote the surface measure on $\partial \Omega$, where as usual $H^{n}$ denotes $n$-dimensional Hausdorff measure. It is well known that in the absence of Reifenberg flatness, $\log k \in V M O(d \sigma)$ need not imply that $\nu \in V M O(d \sigma)$ : the counterexample is the double cone (see $[\mathrm{AC}]$ and $[\mathrm{KP}])$. With this in mind one may ask under what other hypotheses do we have $\log k \in V M O(d \sigma) \Longrightarrow \nu \in V M O(d \sigma)$, and when do we have $\log k \in$ $V M O(d \sigma) \Longleftrightarrow \nu \in V M O(d \sigma) ?$ 
Steve Hofmann, Marius Mitrea and Michael Taylor [HMT], showed that if $\Omega$ is 2-sided NTA, with Ahlfors-David regular ("ADR") boundary, then $\nu \in V M O(d \sigma)$ implies vanishing Reifenberg flatness, and hence that $\log k \in V M O(d \sigma)$, by the result of [KT1]. Thus, it seems reasonable to conjecture that in the setting of a 2-sided NTA domain with ADR boundary, $\log k \in V M O(d \sigma) \Longrightarrow \nu \in V M O(d \sigma)$. Indeed, this very question was posed in [KT4]. In trying to answer this question (which remains open), the author and Steve Hofmann found an alternative approach, based on $L^{p}$ bounds and jump relations for layer potentials, to prove a two-phase version of the problem, which had previously been treated in [KT4].

In [KT4] Kenig and Toro established the following: suppose that $\Omega$ is a 2 -sided chord-arc domain (i.e, a 2-sided NTA domain with ADR boundary), and that $k_{1}$ and $k_{2}$ are the Poisson kernels of $\Omega$ and $\Omega_{\text {ext }}$ respectively, with some fixed poles (either finite or infinite). If $\log k_{1}, \log k_{2} \in V M O(d \sigma)$ (or even $V M O_{l o c}$ ), then $\nu \in$ $V M O_{l o c}(d \sigma)$. Their proof uses a blow-up argument which is quite natural given the nature of the problem. This blow-up argument, as well as our arguments here, require that one work with local versions of $V M O$; see Definition 4.1.16 below. In the present paper, we will use $L^{p}$ bounds and jump relations for the gradient of the single layer potential to show that if $\log k_{1}, \log k_{2} \in V M O_{l o c}(d \sigma)$, then $\nu \in V M O_{l o c}(d \sigma)$. Our approach has the additional modest virtue that it allows us to weaken the 2-sided NTA condition in a non-trivial way, in the case that our Poisson kernels have finite poles.

\subsection{Notation}

Throughout the text the following notation will be used. 
- We will use $\Omega$ to denote an open set in $\mathbb{R}^{n+1}$ with $n \geq 1$. (In Chapter 4 we will assume, among other conditions, that $n \geq 2$.)

- We use the letters $c, C$ to denote harmless positive constants, not necessarily the same at each occurrence, which depend only on dimension and the constants appearing in the hypotheses of the theorems (which we refer to as the "allowable parameters"). We shall also sometimes write $a \lesssim b$ and $a \approx b$ to mean, respectively, that $a \leq C b$ and $0<c \leq a / b \leq C$, where the constants $c$ and $C$ are as above, unless explicitly noted to the contrary. At times, we shall designate by $M$ a particular constant whose value will remain unchanged throughout the proof of a given lemma or proposition, but which may have a different value during the proof of a different lemma or proposition.

- Given a closed set $E \subset \mathbb{R}^{n+1}$ (often the boundary of $\Omega$ ), we shall use lower case letters $x, y, z$, etc., to denote points on $E$, and capital letters $X, Y, Z$, etc., to denote generic points in $\mathbb{R}^{n+1}$ (especially those in $\mathbb{R}^{n+1} \backslash E$ ).

- The open $(n+1)$-dimensional Euclidean ball of radius $r$ will be denoted $B(x, r)$ when the center $x$ lies on $E$, or $B(X, r)$ when the center $X \in(\partial \Omega)^{c}$. A "surface ball" is denoted $\Delta(x, r):=B(x, r) \cap E$.

- Given a (fixed) closed set $E \subset \mathbb{R}^{n+1}$, for $X \in \mathbb{R}^{n+1}$, we set $\delta(X):=\operatorname{dist}(X, E)$.

- Given a Euclidean or surface ball $B=B(X, r)$ or $\Delta=\Delta(x, r)$, its concentric dilate by a factor of $\kappa>0$ will be denoted $\kappa B:=B(X, \kappa r)$ or $\kappa \Delta:=\Delta(x, \kappa r)$.

- We let $H^{n}$ denote $n$-dimensional Hausdorff measure, and let $\sigma:=H^{n}\llcorner E$ denote 
the "surface measure" on a closed set $E \subset \mathbb{R}^{n+1}$ of co-dimension 1 .

- For a Borel set $A \subset \mathbb{R}^{n+1}$, we let $1_{A}$ denote the usual indicator function of $A$, i.e. $1_{A}(x)=1$ if $x \in A$, and $1_{A}(x)=0$ if $x \notin A$.

- For $A \subset \mathbb{R}^{n+1}$, we let $\operatorname{int}(A)$ denote the interior of $A$.

- Given a Borel measure $\mu$, and a Borel set $A$, with positive and finite $\mu$ measure, we set $f_{A} f d \mu:=\mu(A)^{-1} \int_{A} f d \mu$.

- We shall use the letter $I$ (and sometimes $J$ ) to denote a closed $(n+1)$-dimensional Euclidean dyadic cube with sides parallel to the co-ordinate axes, and we let $\ell(I)$ denote the side length of $I$. If $\ell(I)=2^{-k}$, then we set $k_{I}:=k$. Given an ADR set $E \subset \mathbb{R}^{n+1}$, we use $Q$ to denote a dyadic "cube" on $E$. The latter exist (cf. [DS1], $[\mathrm{Chr}])$, and enjoy certain properties which we enumerate in Lemma 2.1.17 below. 


\section{Chapter 2}

\section{Uniform Rectifiability and Harmonic Measure}

In this chapter we explore harmonic measure for open sets with uniformly rectifiable (UR) boundaries. This is part of a larger program to characterize UR sets in terms of harmonic measure. In [HM3] the authors show that if $E \subset \mathbb{R}^{n+1}$ is an ADR set for which the harmonic measure for $\mathbb{R}^{n+1} \backslash E$ is in weak- $A_{\infty}$ with respect to surface measure then $E$ is UR. This result and Theorem 2.4.1 allow one to characterize UR sets by properties of harmonic measure. To be precise we have that an ADR set is UR if and only if it has "Interior Big Pieces of Good Harmonic Estimates" (IBP(GHME)),

see Definition 2.1.16). After this, we show that if $\Omega \subset \mathbb{R}^{n+1}$ is a domain with UR boundary and $\Omega$ satisfies the interior corkscrew condition then surface measure is absolutely continuous with respect to harmonic measure for $\Omega$.

\subsection{Notation and Definitions}

We first recall several definitions.

Definition 2.1.1. (ADR) (aka Ahlfors-David regular). We say that a set $E \subset \mathbb{R}^{n+1}$, of Hausdorff dimension $n$, is ADR if it is closed, and if there is some uniform constant 
$C$ such that

$$
\frac{1}{C} r^{n} \leq \sigma(\Delta(x, r)) \leq C r^{n}, \quad \forall r \in(0, \operatorname{diam}(E)), x \in E
$$

where $\operatorname{diam}(E)$ may be infinite. Here, $\Delta(x, r):=E \cap B(x, r)$ is the "surface ball" of radius $r$, and $\sigma:=H^{n}\left\llcorner E\right.$ is the "surface measure" on $E$, where $H^{n}$ denotes n-dimensional Hausdorff measure.

Definition 2.1.3. (UR) (aka uniformly rectifiable). An n-dimensional ADR (hence closed) set $E \subset \mathbb{R}^{n+1}$ is UR if and only if it contains "Big Pieces of Lipschitz Images" of $\mathbb{R}^{n}$ ("BPLI"). This means that there are positive constants $\theta$ and $M_{0}$, such that for each $x \in E$ and each $r \in(0, \operatorname{diam}(E))$, there is a Lipschitz mapping $\rho=\rho_{x, r}$ : $\mathbb{R}^{n} \rightarrow \mathbb{R}^{n+1}$, with Lipschitz constant no larger than $M_{0}$, such that

$$
H^{n}\left(E \cap B(x, r) \cap \rho\left(\left\{z \in \mathbb{R}^{n}:|z|<r\right\}\right)\right) \geq \theta r^{n}
$$

We recall that $n$-dimensional rectifiable sets are characterized by the property that they can be covered, up to a set of $H^{n}$ measure 0 , by a countable union of Lipschitz images of $\mathbb{R}^{n}$; thus, BPLI is a quantitative version of this fact.

There are numerous other characterizations of UR sets (many of which remain valid in higher co-dimensions), see [DS1, DS2]. In particular, at least among the class of ADR sets, the UR sets are precisely those for which all "sufficiently nice" singular integrals are $L^{2}$-bounded [DS1]. In fact, for $n$-dimensional ADR sets in $\mathbb{R}^{n+1}$, the $L^{2}$ boundedness of certain special singular integral operators (the "Riesz Transforms"), suffices to characterize uniform rectifiability (see [MMV] for the case $n=1$, and $[\mathrm{NToV}]$ in general).

Indeed, for the purposes of Chapter 4, it is most useful to use the following definition. Let $E \subset \mathbb{R}^{n+1}$ be an $n$-dimensional ADR (hence closed) set with surface 
measure $\sigma$. Then $E$ is UR if and only if the Riesz transform operator is $L^{2}$ bounded with respect to surface measure, in the sense that

$$
\sup _{\varepsilon>0}\left\|\mathcal{R}_{\varepsilon} f\right\|_{L^{2}(E, \sigma)} \leq C\|f\|_{L^{2}(E, \sigma)}
$$

See [DS1] for a proof of the fact that UR (defined in various other ways) implies (2.1.4). For the converse, see $[\mathrm{MMV}]$ in the case $n=1$, and $[\mathrm{NToV}]$ in general. In Chapter 4, we shall require only the first implication (UR implies (2.1.4)).

We remark that there exist sets that are ADR (and that even form the boundary of a domain satisfying interior Corkscrew and Harnack Chain conditions), but that are purely unrectifiable (e.g., see the construction of Garnett's "4-corners Cantor set" in [DS2, Chapter1]).

Definition 2.1.5. $\left(B P(\mathcal{S})\right.$ and $\left.B P^{2}(L G)\right)$. Let $\mathcal{S}$ be a collection of subsets of $\mathbb{R}^{n+1}$. We say an $n$-dimensional $A D R$ set $E \subset \mathbb{R}^{n+1}$ has big pieces of $\mathcal{S}$ (" $E \in B P(\mathcal{S})$ ") if there exists a positive constant $\theta$ such that for each $x \in E$ and $r \in(0, \operatorname{diam}(E))$, there is a set $S \in \mathcal{S}$ with

$$
H^{n}(B(x, r) \cap E \cap S) \geq \theta r^{n}
$$

A Lipschitz graph in $\mathbb{R}^{n+1}$ is a set of the form

$$
\{y+\rho(y): y \in P\}
$$

where $P$ is an $n$-plane and $\rho$ is a Lipschitz mapping onto a line perpendicular to $\mathrm{P}$. We say that $E$ has big pieces of Lipschitz graphs (" $B P(L G)$ ") if there exists a positive constant $M_{0}$ such that $E \in B P(\mathcal{S})$, where $\mathcal{S}$ is the collection of all Lipschitz graphs with Lipschitz constant no greater than $M_{0}$. 
Finally, if $E$ has $B P(\mathcal{S})$, where $\mathcal{S}$ is a collection of sets satisfying $B P(L G)$, with uniform bounds on the various constants, then we say that $E \in B P^{2}(L G)$.

Definition 2.1.6. ("UR character"). Given a UR set $E \subset \mathbb{R}^{n+1}$, its "UR character" is just the pair of constants $\left(\theta, M_{0}\right)$ involved in the definition of uniform rectifiability, along with the ADR constant; or equivalently, the quantitative bounds involved in any particular characterization of uniform rectifiability.

Definition 2.1.7. (Corkscrew point and Corkscrew condition). Following [JK], given an open set $\Omega \subset \mathbb{R}^{n+1}$, and a ball $B=B(x, r)$, with $x \in \partial \Omega$ and $0<r<$ $\operatorname{diam}(\partial \Omega)$, we say that a point $X=X_{B} \in \Omega$ is a Corkscrew point relative to $B$ with constant $c>0$, if there is a ball $B(X, c r) \subset B(x, r) \cap \Omega$. If there exists a $c>0$ such that for every $x \in \partial \Omega$ and $r \in(0, \operatorname{diam}(\partial \Omega)$ there exists a corkscrew point relative to $B(x, r)$ with constant $c$, we say $\Omega$ satisfies and interior Corkscrew condition.

Definition 2.1.8. (2-sided Corkscrew condition). We say that an open set $\Omega$ satisfies the 2-sided Corkscrew condition if for some uniform constant $c>0$ (the "Corkscrew constant"), and for every $x \in \partial \Omega$ and $0<r<\operatorname{diam}(\partial \Omega)$, there are two Corkscrew points $X_{1}$ and $X_{2}$ relative to $B(x, r)$, with constant $c$ (as in Definition 2.1.7), and two distinct connected components of $\mathbb{R}^{n} \backslash \partial \Omega, \mathcal{O}_{1}$ and $\mathcal{O}_{2}$, with $B_{1}=$ $B\left(X_{1}, c r\right) \subset \mathcal{O}_{1}$ and $B_{2}=B\left(X_{2}, c r\right) \subset \mathcal{O}_{2}$. We recall that this property is called "Condition B" in the work of David and Semmes [DS3] . We refer to the balls $B_{1}$ and $B_{2}$ as Corkscrew balls.

Definition 2.1.9. (Strong 2-sided Corkscrew condition). We say that an open set $\Omega$ satisfies the strong 2-sided Corkscrew condition if $\Omega$ satisfies the 2 -sided Corkscrew condition, and one of the balls $B_{1} \subset \Omega$ or $B_{2} \subset \Omega$. 
Definition 2.1.10. (Harnack Chain condition). Following [JK], we say that $\Omega$ satisfies the Harnack Chain condition if there is a uniform constant $C$ such that for every $\rho>0, \Lambda \geq 1$, and every pair of points $X, X^{\prime} \in \Omega$ with $\delta(X), \delta\left(X^{\prime}\right) \geq \rho$ and $\left|X-X^{\prime}\right|<\Lambda \rho$, there is a chain of open balls $B_{1}, \ldots, B_{N} \subset \Omega, N \leq C(\Lambda)$, with $X \in B_{1}, X^{\prime} \in B_{N}, B_{k} \cap B_{k+1} \neq \varnothing$ and $C^{-1} \operatorname{diam}\left(B_{k}\right) \leq \operatorname{dist}\left(B_{k}, \partial \Omega\right) \leq C \operatorname{diam}\left(B_{k}\right)$. The chain of balls is called a "Harnack Chain".

Definition 2.1.11. (NTA). Again following [JK], we say that a domain $\Omega \subset \mathbb{R}^{n+1}$ is NTA ("Non-tangentially accessible") if it satisfies the Harnack Chain condition, and the strong 2-sided Corkscrew condition.

Definition 2.1.12. (Chord-arc domain). An NTA domain with an ADR boundary is said to be a Chord-arc domain.

Definition 2.1.13. $\left(A_{\infty}\right.$ and weak- $\left.A_{\infty}\right)$. Given an ADR set $E \subset \mathbb{R}^{n+1}$, and a surface ball $\Delta_{0}:=B_{0} \cap E$, we say that a Borel measure $\mu$ defined on $E$ belongs to $A_{\infty}\left(\Delta_{0}\right)$ if there are positive constants $C$ and $\theta$ such that for each surface ball $\Delta=B \cap E$, with $B \subseteq B_{0}$, we have

$$
\mu(F) \leq C\left(\frac{\sigma(F)}{\sigma(\Delta)}\right)^{\theta} \mu(\Delta), \quad \text { for every Borel set } F \subset \Delta .
$$

Similarly, $\mu \in$ weak- $A_{\infty}\left(\Delta_{0}\right)$, with $\Delta_{0}=B_{0} \cap \partial \Omega$, if for every $\Delta=B \cap \partial \Omega$ with $2 B \subseteq B_{0}$ we have

$$
\mu(F) \leq C\left(\frac{\sigma(F)}{\sigma(\Delta)}\right)^{\theta} \mu(2 \Delta), \quad \text { for every Borel set } F \subset \Delta .
$$

In the case that $\mu=\omega$ is harmonic measure for an open set $\Omega$ satisfying an interior Corkscrew condition, setting $E=\partial \Omega$, we shall say that $\omega$ belongs to $A_{\infty}$ (resp., weak$\left.A_{\infty}\right)$, if for every surface ball $\Delta_{0}$, and for any Corkscrew point $X_{\Delta_{0}} \in \Omega$ relative to $\Delta_{0}$, 
harmonic measure $\omega^{X_{\Delta_{0}}}$, with pole at $X_{\Delta_{0}}$, belongs to $A_{\infty}\left(\Delta_{0}\right)\left(\operatorname{resp}\right.$., weak- $\left.A_{\infty}\left(\Delta_{0}\right)\right)$, in the sense above.

Definition 2.1.16. (IBP(GHME)). When the collection $\mathcal{S}$ in Definition 2.1 .5 consists of boundaries of domains $\widetilde{\Omega} \subset \mathbb{R}^{n+1} \backslash E$, for which the associated harmonic measures belong to weak- $A_{\infty}$, and if the various boundaries $\{\partial \widetilde{\Omega}\}$ are ADR, with uniform control of the $\mathrm{ADR}$ and weak- $A_{\infty}$ constants, then we say that $E$ has "interior big pieces of good harmonic measure estimates", and we write $E \in I B P(G H M E)$.

\section{Lemma 2.1.17. (Existence and properties of the "dyadic grid") [DS1, DS2],} [Chr]. Suppose that $E \subset \mathbb{R}^{n+1}$ is a closed $n$-dimensional ADR set. Then there exist constants $a_{0}>0, \gamma>0$ and $C_{1}<\infty$, depending only on dimension and the $A D R$ constant, such that for each $k \in \mathbb{Z}$, there is a collection of Borel sets ("cubes")

$$
\mathbb{D}_{k}:=\left\{Q_{j}^{k} \subset E: j \in \mathfrak{I}_{k}\right\}
$$

where $\mathfrak{I}_{k}$ denotes some (possibly finite) index set depending on $k$, satisfying

(i) $E=\cup_{j} Q_{j}^{k}$ for each $k \in \mathbb{Z}$.

(ii) If $m \geq k$ then either $Q_{i}^{m} \subset Q_{j}^{k}$ or $Q_{i}^{m} \cap Q_{j}^{k}=\varnothing$.

(iii) For each $(j, k)$ and each $m<k$, there is a unique $i$ such that $Q_{j}^{k} \subset Q_{i}^{m}$.

(iv) $\operatorname{diam}\left(Q_{j}^{k}\right) \leq C_{1} 2^{-k}$

(v) Each $Q_{j}^{k}$ contains some "surface ball" $\Delta\left(x_{j}^{k}, a_{0} 2^{-k}\right):=B\left(x_{j}^{k}, a_{0} 2^{-k}\right) \cap E$.

(vi) $H^{n}\left(\left\{x \in Q_{j}^{k}: \operatorname{dist}\left(x, E \backslash Q_{j}^{k}\right) \leq \varrho 2^{-k}\right\}\right) \leq C_{1} \varrho^{\gamma} H^{n}\left(Q_{j}^{k}\right)$, for all $k, j$ and for all $\varrho \in\left(0, a_{0}\right)$. 
A few remarks are in order concerning this lemma.

- In the setting of a general space of homogeneous type, this lemma has been proved by Christ [Chr], with the dyadic parameter $1 / 2$ replaced by some constant $\delta \in(0,1)$. In fact, one may always take $\delta=1 / 2$ (see [HMMM, Proof of Proposition 2.12]). In the presence of the Ahlfors-David property (2.1.2), the result already appears in [DS1, DS2].

- For our purposes, we may ignore those $k \in \mathbb{Z}$ such that $2^{-k} \gtrsim \operatorname{diam}(E)$, in the case that the latter is finite.

- We shall denote by $\mathbb{D}=\mathbb{D}(E)$ the collection of all relevant $Q_{j}^{k}$, i.e.,

$$
\mathbb{D}:=\cup_{k} \mathbb{D}_{k}
$$

where, if $\operatorname{diam}(E)$ is finite, the union runs over those $k$ such that $2^{-k} \lesssim \operatorname{diam}(E)$.

- Properties $(i v)$ and $(v)$ imply that for each cube $Q \in \mathbb{D}_{k}$, there is a point $x_{Q} \in E$, a Euclidean ball $B\left(x_{Q}, r\right)$ and a surface ball $\Delta\left(x_{Q}, r\right):=B\left(x_{Q}, r\right) \cap E$ such that $r \approx 2^{-k} \approx \operatorname{diam}(Q)$ and

$$
\Delta\left(x_{Q}, r\right) \subset Q \subset \Delta\left(x_{Q}, C r\right)
$$

for some uniform constant $C$. We shall denote this ball and surface ball by

$$
B_{Q}:=B\left(x_{Q}, r\right), \quad \Delta_{Q}:=\Delta\left(x_{Q}, r\right),
$$

and we shall refer to the point $x_{Q}$ as the "center" of $Q$.

- For each cube $Q \in \mathbb{D}$, we let $X_{Q}$ be a corkscrew point relative to $B_{Q}$, and refer to this as a corkscrew point relative to $Q$. Such a corkscrew point exists, since $E$ 
is $n$-dimensional ADR (with the constant $c$ in Definition 2.1.7 depending only on dimension and the ADR constants).

- For a dyadic cube $Q \in \mathbb{D}_{k}$, we shall set $\ell(Q)=2^{-k}$, and we shall refer to this quantity as the "length" of $Q$. Evidently, $\ell(Q) \approx \operatorname{diam}(Q)$.

- For a dyadic cube $Q \in \mathbb{D}$, we let $k(Q)$ denote the "dyadic generation" to which $Q$ belongs, i.e., we set $k=k(Q)$ if $Q \in \mathbb{D}_{k}$; thus, $\ell(Q)=2^{-k(Q)}$.

\subsection{A bilateral corona decomposition and corona type approximation by chord arc domains: Pre- liminaries for Theorem 2.3.1}

In this section, we state a bilateral variant of the "corona decomposition" of David and Semmes [DS1, DS2]. The bilateral version was proved in [HMM], Lemma 2.2. We first recall the notions of "coherency" and "semi-coherency":

Definition 2.2.1. [DS2]. Let $\mathbf{S} \subset \mathbb{D}(E)$. We say that $\mathbf{S}$ is "coherent" if the following conditions hold:

(a) $\mathbf{S}$ contains a unique maximal element $Q(\mathbf{S})$ which contains all other elements of $\mathbf{S}$ as subsets.

(b) If $Q$ belongs to $\mathbf{S}$, and if $Q \subset \widetilde{Q} \subset Q(\mathbf{S})$, then $\widetilde{Q} \in \mathbf{S}$.

(c) Given a cube $Q \in \mathbf{S}$, either all of its children belong to $\mathbf{S}$, or none of them do.

We say that $\mathbf{S}$ is "semi-coherent" if only conditions $(a)$ and $(b)$ hold.

The bilateral "corona decomposition" is as follows. 
Lemma 2.2.2. [HMM, Lemma 2.2]. Suppose that $E \subset \mathbb{R}^{n+1}$ is $n$-dimensional $U R$. Then given any positive constants $\eta \ll 1$ and $K \gg 1$, there is a disjoint decomposition $\mathbb{D}(E)=\mathcal{G} \cup \mathcal{B}$, satisfying the following properties.

1. The "Good" collection $\mathcal{G}$ is further subdivided into disjoint stopping time regimes, such that each such regime $\mathbf{S}$ is coherent (cf. Definition 2.2.1).

2. The "Bad" cubes, as well as the maximal cubes $Q(\mathbf{S})$ satisfy a Carleson packing condition:

$$
\sum_{Q^{\prime} \subset Q, Q^{\prime} \in \mathcal{B}} \sigma\left(Q^{\prime}\right)+\sum_{\mathbf{S}: Q(\mathbf{S}) \subset Q} \sigma(Q(\mathbf{S})) \leq C_{\eta, K} \sigma(Q), \quad \forall Q \in \mathbb{D}(E) .
$$

3. For each $\mathbf{S}$, there is a Lipschitz graph $\Gamma_{\mathbf{S}}$, with Lipschitz constant at most $\eta$, such that, for every $Q \in \mathbf{S}$,

$$
\sup _{x \in \Delta_{Q}^{*}} \operatorname{dist}\left(x, \Gamma_{\mathbf{S}}\right)+\sup _{y \in B_{Q}^{*} \cap \Gamma_{\mathbf{S}}} \operatorname{dist}(y, E)<\eta \ell(Q),
$$

where $B_{Q}^{*}:=B\left(x_{Q}, K \ell(Q)\right)$ and $\Delta_{Q}^{*}:=B_{Q}^{*} \cap E$.

In this section, we construct the same domains as in [HMM], for each stopping time regime $\mathbf{S}$ in Lemma 2.2.2, a pair of NTA domains $\Omega_{\mathbf{S}}^{ \pm}$, with ADR boundaries, which provide a good approximation to $E$, at the scales within $\mathbf{S}$, in some appropriate sense. To be a bit more precise, $\Omega_{\mathbf{S}}:=\Omega_{\mathbf{S}}^{+} \cup \Omega_{\mathbf{S}}^{-}$will be constructed as a sawtooth region relative to some family of dyadic cubes, and the nature of this construction will be essential to the dyadic analysis that we will use below. In this section, we follow essentially verbatim the construction in [HMM], which we reproduce here for the reader's convenience.

We first discuss some preliminary matters. We shall utilize the notation and constructions of [HMM] (and essentially that of [HM2] and [HMU]). 
Let $\mathcal{W}=\mathcal{W}\left(\mathbb{R}^{n+1} \backslash E\right)$ denote a collection of (closed) dyadic Whitney cubes of $\mathbb{R}^{n+1} \backslash E$, so that the cubes in $\mathcal{W}$ have pairwise disjoint interiors and cover $\mathbb{R}^{n+1} \backslash E$, and furthermore satisfy

$$
4 \operatorname{diam}(I) \leq \operatorname{dist}(4 I, E) \leq \operatorname{dist}(I, E) \leq 40 \operatorname{diam}(I), \quad \forall I \in \mathcal{W}
$$

(just dyadically divide the standard Whitney cubes, as constructed in [Ste, Chapter VI], into cubes with side length $1 / 8$ as large) and also

$$
(1 / 4) \operatorname{diam}\left(I_{1}\right) \leq \operatorname{diam}\left(I_{2}\right) \leq 4 \operatorname{diam}\left(I_{1}\right)
$$

whenever $I_{1}$ and $I_{2}$ touch.

Let $E$ be an $n$-dimensional ADR set and pick two parameters $\eta \ll 1$ and $K \gg 1$. Define

$$
\mathcal{W}_{Q}^{0}:=\left\{I \in \mathcal{W}: \eta^{1 / 4} \ell(Q) \leq \ell(I) \leq K^{1 / 2} \ell(Q), \operatorname{dist}(I, Q) \leq K^{1 / 2} \ell(Q)\right\}
$$

Remark 2.2.6. We note that $\mathcal{W}_{Q}^{0}$ is non-empty, provided that we choose $\eta$ small enough, and $K$ large enough, depending only on dimension and the ADR constant of E.

Assume now that $E$ is UR and make the corresponding bilateral corona decomposition of Lemma 2.2.2 with $\eta \ll 1$ and $K \gg 1$. Given $Q \in \mathbb{D}(E)$, for this choice of $\eta$ and $K$, we set (as above) $B_{Q}^{*}:=B\left(x_{Q}, K \ell(Q)\right)$, where we recall that $x_{Q}$ is the "center" of $Q$ (see (2.1.18)-(2.1.19)). For a fixed stopping time regime $\mathbf{S}$, we choose a co-ordinate system so that $\Gamma_{\mathbf{S}}=\left\{\left(z, \varphi_{\mathbf{S}}(z)\right): z \in \mathbb{R}^{n}\right\}$, where $\varphi_{\mathbf{S}}: \mathbb{R}^{n} \mapsto \mathbb{R}$ is a Lipschitz function with $\|\varphi\|_{\text {Lip }} \leq \eta$. 
Claim 2.2.7. If $Q \in \mathbf{S}$, and $I \in \mathcal{W}_{Q}^{0}$, then $I$ lies either above or below $\Gamma_{\mathbf{S}}$. Moreover, $\operatorname{dist}\left(I, \Gamma_{\mathbf{S}}\right) \geq \eta^{1 / 2} \ell(Q)$ (and therefore, by $(2.2 .3), \operatorname{dist}\left(I, \Gamma_{\mathbf{S}}\right) \approx \operatorname{dist}(I, E)$, with implicit constants that may depend on $\eta$ and $K$ ).

Proof of Claim 2.2.7. Suppose by way of contradiction that $\operatorname{dist}\left(I, \Gamma_{\mathbf{S}}\right) \leq \eta^{1 / 2} \ell(Q)$. Then we may choose $y \in \Gamma_{\mathbf{S}}$ such that

$$
\operatorname{dist}(I, y) \leq \eta^{1 / 2} \ell(Q)
$$

By construction of $\mathcal{W}_{Q}^{0}$, it follows that for all $Z \in I,|Z-y| \lesssim K^{1 / 2} \ell(Q)$. Moreover, $\left|Z-x_{Q}\right| \lesssim K^{1 / 2} \ell(Q)$, and therefore $\left|y-x_{Q}\right| \lesssim K^{1 / 2} \ell(Q)$. In particular, $y \in B_{Q}^{*} \cap \Gamma_{\mathbf{S}}$, so by $(2.2 .3), \operatorname{dist}(y, E) \leq \eta \ell(Q)$. On the other hand, choosing $Z_{0} \in I$ such that $\left|Z_{0}-y\right|=\operatorname{dist}(I, y) \leq \eta^{1 / 2} \ell(Q)$, we obtain $\operatorname{dist}(I, E) \leq 2 \eta^{1 / 2} \ell(Q)$. For $\eta$ small, this contradicts the Whitney construction, since $\operatorname{dist}(I, E) \approx \ell(I) \geq \eta^{1 / 4} \ell(Q)$.

Next, given $Q \in \mathbf{S}$, we augment $\mathcal{W}_{Q}^{0}$. We split $\mathcal{W}_{Q}^{0}=\mathcal{W}_{Q}^{0,+} \cup \mathcal{W}_{Q}^{0,-}$, where $I \in \mathcal{W}_{Q}^{0,+}$ if $I$ lies above $\Gamma_{\mathbf{S}}$, and $I \in \mathcal{W}_{Q}^{0,-}$ if $I$ lies below $\Gamma_{\mathbf{S}}$. Choosing $K$ large and $\eta$ small enough, by (2.2.3), we may assume that both $\mathcal{W}_{Q}^{0, \pm}$ are non-empty. We focus on $\mathcal{W}_{Q}^{0,+}$, as the construction for $\mathcal{W}_{Q}^{0,-}$ is the same. For each $I \in \mathcal{W}_{Q}^{0,+}$, let $X_{I}$ denote the center of $I$. Fix one particular $I_{0} \in \mathcal{W}_{Q}^{0,+}$, with center $X_{Q}^{+}:=X_{I_{0}}$. Let $\widetilde{Q}$ denote the dyadic parent of $Q$, unless $Q=Q(\mathbf{S})$; in the latter case we simply set $\widetilde{Q}=Q$. Note that $\widetilde{Q} \in \mathbf{S}$, by the coherency of $\mathbf{S}$. By Claim 2.2 .7 , for each $I$ in $\mathcal{W}_{Q}^{0,+}$, or in $\mathcal{W}_{\widetilde{Q}}^{0,+}$, we have

$$
\operatorname{dist}(I, E) \approx \operatorname{dist}(I, Q) \approx \operatorname{dist}\left(I, \Gamma_{\mathbf{S}}\right),
$$

where the implicit constants may depend on $\eta$ and $K$. Thus, for each such $I$, we may 
fix a Harnack chain, call it $\mathcal{H}_{I}$, relative to the Lipschitz domain

$$
\Omega_{\Gamma_{\mathbf{S}}}^{+}:=\left\{(x, t) \in \mathbb{R}^{n+1}: t>\varphi_{\mathbf{S}}(x)\right\}
$$

connecting $X_{I}$ to $X_{Q}^{+}$. By the bilateral approximation condition (2.2.3), the definition of $\mathcal{W}_{Q}^{0}$, and the fact that $K^{1 / 2} \ll K$, we may construct this Harnack Chain so that it consists of a bounded number of balls (depending on $\eta$ and $K$ ), and stays a distance at least $c \eta^{1 / 2} \ell(Q)$ away from $\Gamma_{\mathbf{S}}$ and from $E$. We let $\mathcal{W}_{Q}^{*,+}$ denote the set of all $J \in \mathcal{W}$ which meet at least one of the Harnack chains $\mathcal{H}_{I}$, with $I \in \mathcal{W}_{Q}^{0,+} \cup \mathcal{W}_{\widetilde{Q}}^{0,+}$ (or simply $I \in \mathcal{W}_{Q}^{0,+}$, if $\left.Q=Q(\mathbf{S})\right)$, i.e.,

$$
\mathcal{W}_{Q}^{*,+}:=\left\{J \in \mathcal{W}: \exists I \in \mathcal{W}_{Q}^{0,+} \cup \mathcal{W}_{\widetilde{Q}}^{0,+} \text { for which } \mathcal{H}_{I} \cap J \neq \varnothing\right\},
$$

where as above, $\widetilde{Q}$ is the dyadic parent of $Q$, unless $Q=Q(\mathbf{S})$, in which case we simply set $\widetilde{Q}=Q$ (so the union is redundant). We observe that, in particular, each $I \in \mathcal{W}_{Q}^{0,+} \cup \mathcal{W}_{\widetilde{Q}}^{0,+}$ meets $\mathcal{H}_{I}$, by definition, and therefore

$$
\mathcal{W}_{Q}^{0,+} \cup \mathcal{W}_{\widetilde{Q}}^{0,+} \subset \mathcal{W}_{Q}^{*,+}
$$

Of course, we may construct $\mathcal{W}_{Q}^{*,-}$ analogously. We then set

$$
\mathcal{W}_{Q}^{*}:=\mathcal{W}_{Q}^{*,+} \cup \mathcal{W}_{Q}^{*,-}
$$

It follows from the construction of the augmented collections $\mathcal{W}_{Q}^{*, \pm}$ that there are uniform constants $c$ and $C$ such that

$$
\begin{gathered}
c \eta^{1 / 2} \ell(Q) \leq \ell(I) \leq C K^{1 / 2} \ell(Q), \quad \forall I \in \mathcal{W}_{Q}^{*}, \\
\operatorname{dist}(I, Q) \leq C K^{1 / 2} \ell(Q), \quad \forall I \in \mathcal{W}_{Q}^{*} .
\end{gathered}
$$

Observe that $\mathcal{W}_{Q}^{*, \pm}$ and hence also $\mathcal{W}_{Q}^{*}$ have been defined for any $Q$ that belongs to some stopping time regime $\mathbf{S}$, that is, for any $Q$ belonging to the "good" collection $\mathcal{G}$ of Lemma 2.2.2. On the other hand, we have defined $\mathcal{W}_{Q}^{0}$ for arbitrary $Q \in \mathbb{D}(E)$. 
We now set

$$
\mathcal{W}_{Q}:=\left\{\begin{array}{l}
\mathcal{W}_{Q}^{*}, Q \in \mathcal{G}, \\
\mathcal{W}_{Q}^{0}, Q \in \mathcal{B}
\end{array},\right.
$$

and for $Q \in \mathcal{G}$ we shall henceforth simply write $\mathcal{W}_{Q}^{ \pm}$in place of $\mathcal{W}_{Q}^{*, \pm}$.

Next, we choose a small parameter $\tau_{0}>0$, so that for any $I \in \mathcal{W}$, and any $\tau \in\left(0, \tau_{0}\right]$, the concentric dilate $I^{*}(\tau):=(1+\tau) I$ still satisfies the Whitney property

$$
\operatorname{diam} I \approx \operatorname{diam} I^{*}(\tau) \approx \operatorname{dist}\left(I^{*}(\tau), E\right) \approx \operatorname{dist}(I, E), \quad 0<\tau \leq \tau_{0}
$$

Moreover, for $\tau \leq \tau_{0}$ small enough, and for any $I, J \in \mathcal{W}$, we have that $I^{*}(\tau)$ meets $J^{*}(\tau)$ if and only if $I$ and $J$ have a boundary point in common, and that, if $I \neq J$ then $I^{*}(\tau)$ misses $(3 / 4) J$. Given an arbitrary $Q \in \mathbb{D}(E)$, we may define an associated Whitney region $U_{Q}$ (not necessarily connected), as follows:

$$
U_{Q}=U_{Q, \tau}:=\bigcup_{I \in \mathcal{W}_{Q}} I^{*}(\tau)
$$

If $Q \in \mathcal{G}$, then $U_{Q}$ splits into exactly two connected components

$$
U_{Q}^{ \pm}=U_{Q, \tau}^{ \pm}:=\bigcup_{I \in \mathcal{W}_{Q}^{ \pm}} I^{*}(\tau)
$$

When the particular choice of $\tau \in\left(0, \tau_{0}\right]$ is not important, for the sake of notational convenience, we may simply write $I^{*}, U_{Q}$, and $U_{Q}^{ \pm}$in place of $I^{*}(\tau), U_{Q, \tau}$, and $U_{Q, \tau}^{ \pm}$. We note that for $Q \in \mathcal{G}$, each $U_{Q}^{ \pm}$is Harnack chain connected, by construction (with constants depending on the implicit parameters $\tau, \eta$ and $K)$; moreover, for a fixed stopping time regime $\mathbf{S}$, we claim that if $Q^{\prime}$ is a child of $Q$, with both $Q^{\prime}, Q \in \mathbf{S}$, then $U_{Q^{\prime}}^{+} \cup U_{Q}^{+}$is Harnack Chain connected, and similarly for $U_{Q^{\prime}}^{-} \cup U_{Q}^{-}$. Indeed, by construction (see (2.2.8), the ensuing discussion, and (2.2.9), as well as (2.2.12) and (2.2.14)), each of $U_{Q^{\prime}}^{+}$and $U_{Q}^{+}$is a union of fattened Whitney boxes, such that the 
center of each such fattened Whitney box $I^{*}$ may be connected via a Harnack path to $X_{Q}^{+}$; moreover, any two such $I_{1}^{*}$ and $I_{2}^{*}$ either overlap, or are separated by a distance comparable to $\ell(Q)$. The claimed Harnack Chain property follows readily.

We may also define "Carleson Boxes" relative to any $Q \in \mathbb{D}(E)$, by

$$
T_{Q}=T_{Q, \tau}:=\operatorname{int}\left(\bigcup_{Q^{\prime} \in \mathbb{D}_{Q}} U_{Q, \tau}\right)
$$

where

$$
\mathbb{D}_{Q}:=\left\{Q^{\prime} \in \mathbb{D}(E): Q^{\prime} \subset Q\right\} .
$$

Let us note that we may choose $K$ large enough so that, for every $Q$,

$$
T_{Q} \subset B_{Q}^{*}:=B\left(x_{Q}, K \ell(Q)\right) .
$$

For future reference, we also introduce dyadic sawtooth regions as follows. Given a family $\mathcal{F}$ of disjoint cubes $\left\{Q_{j}\right\} \subset \mathbb{D}$, we define the "global discretized sawtooth" relative to $\mathcal{F}$ by

$$
\mathbb{D}_{\mathcal{F}}:=\mathbb{D} \backslash \bigcup_{\mathcal{F}} \mathbb{D}_{Q_{j}}
$$

i.e., $\mathbb{D}_{\mathcal{F}}$ is the collection of all $Q \in \mathbb{D}$ that are not contained in any $Q_{j} \in \mathcal{F}$. Given some fixed cube $Q$, the "local discretized sawtooth" relative to $\mathcal{F}$ by

$$
\mathbb{D}_{\mathcal{F}, Q}:=\mathbb{D}_{Q} \backslash \bigcup_{\mathcal{F}} \mathbb{D}_{Q_{j}}=\mathbb{D}_{\mathcal{F}} \cap \mathbb{D}_{Q}
$$

Note that in this way $\mathbb{D}_{Q}=\mathbb{D}_{\varnothing, Q}$.

Similarly, we may define geometric sawtooth regions as follows. Given a family $\mathcal{F}$ of disjoint cubes $\left\{Q_{j}\right\} \subset \mathbb{D}$, we define the "global sawtooth" and the "local sawtooth" relative to $\mathcal{F}$ by respectively

$$
\Omega_{\mathcal{F}}:=\operatorname{int}\left(\bigcup_{Q^{\prime} \in \mathbb{D}_{\mathcal{F}}} U_{Q^{\prime}}\right), \quad \Omega_{\mathcal{F}, Q}:=\operatorname{int}\left(\bigcup_{Q^{\prime} \in \mathbb{D}_{\mathcal{F}, Q}} U_{Q^{\prime}}\right)
$$


Notice that $\Omega_{\emptyset, Q}=T_{Q}$.

Remark 2.2.22. Given a stopping time regime $\mathbf{S}$ as in Lemma 2.2.2, for any semicoherent subregime (cf. Definition 2.2.1) $\mathbf{S}^{\prime} \subset \mathbf{S}$ (including, of course, $\mathbf{S}$ itself), we now set

$$
\Omega_{\mathbf{S}^{\prime}}^{ \pm}=\operatorname{int}\left(\bigcup_{Q \in \mathbf{S}^{\prime}} U_{Q}^{ \pm}\right)
$$

and let $\Omega_{\mathbf{S}^{\prime}}:=\Omega_{\mathbf{S}^{\prime}}^{+} \cup \Omega_{\mathbf{S}^{\prime}}^{-}$. Note that implicitly, $\Omega_{\mathbf{S}^{\prime}}$ depends upon $\tau$ (since $U_{Q}^{ \pm}$has such dependence). When it is necessary to consider the value of $\tau$ explicitly, we shall write $\Omega_{\mathbf{S}^{\prime}}(\tau)$.

It is helpful to introduce some terminology now whose utility will become clear later. Let $Q \in \mathbb{D}$ define the following

$$
\mathcal{I}(Q):=\left\{I \in \mathcal{W}: I \cap T_{Q} \neq \varnothing\right\}
$$

and also

$$
V(Q)=\operatorname{int}\left(\bigcup_{I \in \mathcal{I}(Q)} I^{*}\right) .
$$

We note that, trivially, $T_{Q} \subset V(Q)$. Notice also that if $\operatorname{int}\left(I^{*}\right) \subset V(Q)$ then

$$
\operatorname{dist}\left(I^{*}, Q\right) \lesssim \ell(Q)
$$

and

$$
\ell(I) \lesssim \ell(Q)
$$

Lemma 2.2.28. Let $Q_{1}, Q_{2} \in \mathbb{D}(E)$ if $U_{Q_{1}}$ meets $U_{Q_{2}}$ then

$$
\operatorname{dist}\left(Q_{1}, Q_{2}\right) \lesssim \min \left\{\ell\left(Q_{1}\right), \ell\left(Q_{2}\right)\right\}
$$


with implicit constant depending only on $K, \eta$, and dimension. Moreover there exists a constant $\Upsilon$ depending only on $K, \eta$, and dimension such that if

$$
\operatorname{dist}\left(Q_{1}, Q_{2}\right)>\Upsilon \max \left\{\ell\left(Q_{1}\right), \ell\left(Q_{2}\right)\right\}
$$

then

$$
\overline{V\left(Q_{1}\right)} \cap \overline{V\left(Q_{2}\right)}=\varnothing .
$$

Proof. Suppose that $U_{Q_{1}}$ meets $U_{Q_{2}}$ then we have that there exists a cube $I_{1}^{*} \in U_{Q_{1}}$ and $I_{2}^{*} \in U_{Q_{2}}$ such that $I_{1}^{*} \cap I_{2}^{*} \neq \varnothing$. Since $I_{1}^{*}$ and $I_{2}^{*}$ are Whitney cubes that meet we have that

$$
\ell\left(I_{1}\right) \approx \ell\left(I_{2}\right) .
$$

Then by construction of $U_{Q_{1}}$ and $U_{Q_{2}^{\prime}}$ we have

$$
\begin{gathered}
\ell\left(Q_{1}\right) \approx \ell\left(Q_{2}\right), \\
\operatorname{dist}\left(Q_{1}, I_{1}^{*}\right) \lesssim \ell\left(Q_{1}\right), \\
\operatorname{dist}\left(Q_{2}, I_{2}^{*}\right) \lesssim \ell\left(Q_{2}^{*}\right) .
\end{gathered}
$$

So that (2.2.31), (2.2.32), (2.2.33), and (2.2.34) yield

$$
\operatorname{dist}\left(Q_{1}, Q_{2}\right) \lesssim \ell\left(Q_{1}\right)
$$

To prove (2.2.30) we need only see that by (2.2.26) and (2.2.27) if $\overline{V\left(Q_{1}\right)}$ meets $\overline{V\left(Q_{2}\right)}$ that

$$
\operatorname{dist}\left(Q_{1}, Q_{2}\right) \lesssim \max \left\{\ell\left(Q_{1}\right), \ell\left(Q_{2}\right)\right\} \text {. }
$$

In addition we can even put a distance between $V\left(Q_{1}\right)$ and $V\left(Q_{2}\right)$ on the order of $\max \left\{\ell\left(Q_{1}\right), \ell\left(Q_{2}\right)\right\}$ by making $\Upsilon$ larger. 


\subsection{Carleson measures and Theorem 2.3.1}

The objective of this section is to prove the following.

Theorem 2.3.1. Let $E \subset \mathbb{R}^{n+1}$ be a UR set of dimension $n$. Then for each $Q \in \mathbb{D}(E)$

there exists an open set $\widetilde{\Omega}=\widetilde{\Omega}_{Q} \subset \Omega:=\mathbb{R}^{n+1} \backslash E$, with $\operatorname{diam}(\widetilde{\Omega}) \approx \operatorname{diam}(Q)$, such that $\widetilde{\Omega}$ has an ADR (Ahlfors-David Regular) boundary, satisfies a 2-sided corkscrew condition, and

$$
\sigma(\partial \widetilde{\Omega} \cap Q) \gtrsim \sigma(Q)
$$

Moreover, each connected component of $\widetilde{\Omega}$ is an NTA domain with ADR boundary. The various NTA, ADR, and implicit constants are uniformly controlled, and depend only on dimension and on the UR character of $E$.

We remark that, in particular, Theorem 2.3.1 says that $E$ has big pieces of sets satisfying a 2-sided corkscrew condition, and thus, by a result of David and Jerison [DJ] (see also [DS3]), has $B P^{2}(L G)$ (recall Definition 2.1.5). Theorem 2.3.1 therefore yields as an immediate corollary the co-dimension 1 case of a result of Azzam and Schul $[A S]$.

Corollary 2.3.3. Let $E \subset \mathbb{R}^{n+1}$ be a $U R$ set of co-dimension 1. Then $E \in B P^{2}(L G)$.

We should note that, in fact, the result of [AS] establishes $B P^{2}(L G)$ for $U R$ sets in all co-dimensions, whereas our arguments do not address the case of co-dimension greater than 1. On the other hand, in the co-dimension 1 case, our Theorem 2.3.1 yields extra structure which allows us to obtain estimates for harmonic measure. We will explore this in the next section. 
The proof will utilize the method of "extrapolation of Carleson measures". This method was first used by J. L. Lewis [LM], whose work was influenced by the Corona construction of Carleson [Car] and the work of Carleson and Garnett [CG] (see also [HL], [AHLT], [AHMTT], [HM1], [HM2].) We will apply this method to the (discrete) packing measure from the bilateral Corona decomposition. Let $E \subset \mathbb{R}^{n+1}$ be a UR set of co-dimension 1 . We fix positive numbers $\eta \ll 1$, and $K \gg 1$, and for these values of $\eta$ and $K$, we perform the bilateral Corona decomposition of $\mathbb{D}(E)$ guaranteed by Lemma 2.2.2. Let $\mathcal{M}:=\{Q(\mathbf{S})\}_{\mathbf{S}}$ denotes the collection of cubes which are the maximal elements of the stopping time regimes in $\mathcal{G}$. Given a cube $Q \in \mathbb{D}(E)$, we set

$$
\alpha_{Q}:= \begin{cases}\sigma(Q), & \text { if } Q \in \mathcal{M} \cup \mathcal{B}, \\ 0, & \text { otherwise }\end{cases}
$$

Given any collection $\mathbb{D}^{\prime} \subset \mathbb{D}(E)$, we define

$$
\mathfrak{m}\left(\mathbb{D}^{\prime}\right):=\sum_{Q \in \mathbb{D}^{\prime}} \alpha_{Q} .
$$

We recall that $\mathbb{D}_{Q}$ is the "discrete Carleson region relative to $Q$ ", defined in (2.2.17). Then by Lemma 2.2.2 (2), we have the discrete Carleson measure estimate

$$
\begin{aligned}
\mathfrak{m}\left(\mathbb{D}_{Q}\right):=\sum_{Q^{\prime} \subset Q, Q^{\prime} \in \mathcal{B}} \sigma\left(Q^{\prime}\right)+\sum_{\mathbf{S}: Q(\mathbf{S}) \subset Q} \sigma(Q(\mathbf{S})) \leq C_{\eta, K} \sigma(Q), & \\
& \forall Q \in \mathbb{D}(E) .
\end{aligned}
$$

Given a family $\mathcal{F}:=\left\{Q_{j}\right\} \subset \mathbb{D}(E)$ of pairwise disjoint cubes, we recall that the "discrete sawtooth" $\mathbb{D}_{\mathcal{F}}$ is the collection of all cubes in $\mathbb{D}(E)$ that are not contained in any $Q_{j} \in \mathcal{F}$ (cf. (2.2.19)), and we define the "restriction of $\mathfrak{m}$ to the sawtooth $\mathbb{D}_{\mathcal{F}}$ " by

$$
\mathfrak{m}_{\mathcal{F}}\left(\mathbb{D}^{\prime}\right):=\mathfrak{m}\left(\mathbb{D}^{\prime} \cap \mathbb{D}_{\mathcal{F}}\right)=\sum_{Q \in \mathbb{D}^{\prime} \backslash\left(\cup_{\mathcal{F}} \mathbb{D}_{Q_{j}}\right)} \alpha_{Q}
$$


We define the usual Carleson norm, and the Carleson norm restricted to a cube $Q$, as follows

$$
\|\mathfrak{m}\|_{\mathcal{C}}:=\sup _{Q \in \mathbb{D}(E)} \frac{\mathfrak{m}\left(\mathbb{D}_{Q}\right)}{\sigma(Q)}, \quad\|\mathfrak{m}\|_{\mathcal{C}(Q)}:=\sup _{Q^{\prime} \subset Q} \frac{\mathfrak{m}\left(\mathbb{D}_{Q^{\prime}}\right)}{\sigma\left(Q^{\prime}\right)} .
$$

Note that by the way that we have defined $\mathfrak{m}$, we have

$$
\|\mathfrak{m}\|_{\mathcal{C}} \leq C_{\eta, K}
$$

The following Lemma will be one of two crucial lemmas in proving Theorem 2.3.1.

Lemma 2.3.9. [HM2, Lemma 7.2] Suppose that $E$ is $A D R$. Fix $Q \in \mathbb{D}(E)$ and $\mathfrak{m}$ as above. Let $a \geq 0$ and $b>0$, and suppose that $\mathfrak{m}\left(\mathbb{D}_{Q}\right) \leq(a+b) \sigma(Q)$. Then there is a family $\mathcal{F}=\left\{Q_{j}\right\} \subset \mathbb{D}_{Q}$ of pairwise disjoint cubes, and a constant $C$ depending only on dimension and the ADR constant such that

$$
\left\|\mathfrak{m}_{\mathcal{F}}\right\|_{\mathcal{C}(Q)} \leq C b,
$$

and

$$
\sigma(B) \leq \frac{a+b}{a+2 b} \sigma(Q),
$$

where $B$ is the union of those $Q_{j} \in \mathcal{F}$ such that $\mathfrak{m}\left(\mathbb{D}_{Q_{j}} \backslash\left\{Q_{j}\right\}\right)>a \sigma\left(Q_{j}\right)$.

The other crucial lemma is the following.

Lemma 2.3.12. [HMM, Lemma 3.24] : Let $\mathbf{S}$ be a given stopping time regime as in Lemma 2.2.2, and let $\mathbf{S}^{\prime}$ be any nonempty, semi-coherent subregime of $\mathbf{S}$. Then for $0<\tau \leq \tau_{0}$, with $\tau_{0}$ small enough, each of $\Omega_{\mathbf{S}^{\prime}}^{ \pm}$is an NTA domain, with ADR boundary. The constants in the NTA and ADR conditions depend only on $n, \tau, \eta, K$, and the $A D R / U R$ constants for $E$.

The following standard covering type lemma will be required. 
Lemma 2.3.13. Fix $Q_{0} \in \mathbb{D}(E)$ and let $\mathcal{F}=\left\{Q_{j}\right\} \subset \mathbb{D}_{Q_{0}}$ be any pairwise disjoint family of cubes. Then for any positive constant $\kappa$ we may find a sub-collection $\mathcal{G}=$ $\left\{\widetilde{Q}_{i}\right\} \subset \mathcal{F}$ with the following properties:

$$
\begin{gathered}
\sigma\left(\cup_{\mathcal{G}} \widetilde{Q}_{i}\right) \geq C \sigma\left(\cup_{\mathcal{F}} Q_{j}\right) \\
\operatorname{dist}\left(\widetilde{Q}_{i}, \widetilde{Q}_{k}\right) \geq \kappa \max \left\{\ell\left(\widetilde{Q}_{i}\right), \ell\left(\widetilde{Q}_{k}\right)\right)
\end{gathered}
$$

where $C$ depends on $\kappa$, dimension and ADR. Moreover, the collection may be taken to be finite.

We now have the necessary tools to prove Theorem 2.3.1.

Proof of Theorem 2.3.1. First we fix $\eta$ and $K$ so that that Lemma 2.3.12 holds. The proof will follow by induction. For any $a \geq 0$ we have the induction hypothesis $H(a)$, defined in the following way.

$H(a)$ : There exists $\eta_{a}>0$ such that for all $Q_{0} \in \mathbb{D}(E)$ satisfying $\mathfrak{m}\left(\mathbb{D}_{Q_{0}}\right) \leq a \sigma\left(Q_{0}\right)$, there is a collection $\mathbb{D}^{\prime} \subseteq \mathbb{D}_{Q_{0}}$, and an open set $\widetilde{\Omega}$ of the form

$$
\widetilde{\Omega}:=\operatorname{int}\left(\cup_{Q_{j} \in \mathbb{D}^{\prime}} U_{Q_{j}}\right) \subset T_{Q_{0}}
$$

which has an ADR boundary and satisfies the strong 2-sided Corkscrew condition for open sets with Corkscrew balls lying in $V\left(Q_{0}\right)$ (see (2.2.25)), and in addition

$$
\sigma\left(\partial \widetilde{\Omega} \cap Q_{0}\right) \geq \eta_{a} \sigma\left(Q_{0}\right)
$$

Moreover, $\widetilde{\Omega}$ has finitely many connected components and each connected component of $\widetilde{\Omega}$ is an NTA domain with ADR boundary with uniform constants possibly depending on $a$. 
To prove the Theorem it is enough to show that $H\left(M_{0}\right)$ holds where $M_{0}$ is the Carleson norm of $\mathfrak{m}$, that is, $M_{0}=C_{\eta, K}$ in Lemma 2.2.2. We do this by showing first that $H(0)$ holds and then that $H(a)$ implies $H(a+b)$ for some fixed constant $b$ depending only on dimension and the ADR constants. This way we will only use finitely many steps to get to $H\left(M_{0}\right)$. We set $b=\frac{\gamma}{C}$ where $C$ is the constant in (2.3.10) and $\gamma$ is a small positive number to be chosen.

The fact that $H(0)$ holds is somewhat trivial since this would imply that $Q_{0}$ and all of its descendants are in the same stopping time regime and therefore we can directly apply the results in $[\mathrm{HMM}]$, that is, Lemma 2.3.12. We are then left with showing that $H(a)$ implies $H(a+b)$.

Proof that $H(a) \Longrightarrow H(a+b)$ : Suppose that $H(a)$ holds. Let $Q_{0} \in \mathbb{D}(E)$ be such that $\mathfrak{m}\left(\mathbb{D}_{Q_{0}}\right) \leq(a+b) \sigma\left(Q_{0}\right)$. First let $C_{2}$ be an integer so large that if $Q_{1} \subseteq Q_{2}$ with $k\left(Q_{2}\right)+C_{2}-5<k\left(Q_{1}\right)$ then we have that $U_{Q_{2}} \cap V\left(Q_{1}\right)=\varnothing$. We obtain via Lemma 2.3.9 a collection $\mathcal{F}=\left\{Q_{j}\right\}_{j=1}^{\infty}$ such that

$$
\left\|\mathfrak{m}_{\mathcal{F}}\right\|_{C\left(Q_{0}\right)} \leq C b=\gamma
$$

and

$$
\sigma(B) \leq \frac{a+b}{a+2 b} \sigma\left(Q_{0}\right),
$$

where $B$ is the union of those $Q_{j}$ in $\mathcal{F}$ such that $\mathfrak{m}\left(\mathbb{D}_{Q_{j}} \backslash\left\{Q_{j}\right\}\right)>a \sigma\left(Q_{j}\right)$, call this collection $\mathcal{F}_{\text {bad }}$. Define $\mathcal{F}_{\text {good }}:=\mathcal{F} \backslash \mathcal{F}_{\text {bad }}$. Then by pigeon-holing, for each $Q_{j} \in \mathcal{F}_{\text {good }}$ we may find a child of $Q_{j}$ to which we can apply the induction hypothesis $H(a)$. Iterating the pigeon-holing argument, we may find a cube $Q_{j}^{\prime}$ that is $C_{2}$ generations down from $Q_{j}$ (i.e., so that $\ell\left(Q_{j}^{\prime}\right)=2^{-C_{2}} \ell\left(Q_{j}\right)$ ), to which we may apply the induction hypothesis. 
Remark 2.3.20. Choosing $\gamma$ small enough in (2.3.18) (in fact, $\gamma=1 / 2$ will suffice), we obtain that $\mathbb{D}_{\mathcal{F}, Q_{0}}$ does not contain any $Q^{\prime} \in \mathcal{M} \cup \mathcal{B}$, where $\mathcal{M}:=\{Q(\mathbf{S})\}_{\mathbf{S}}$. Fixing such a $\gamma$, we find therefore that every $Q^{\prime} \in \mathbb{D}_{\mathcal{F}, Q_{0}}$ is a good cube, and moreover all such $Q^{\prime}$ belong to the same $\mathbf{S}$, a stopping time regime as in Lemma 2.2.2. Thus, $\mathbb{D}_{\mathcal{F}, Q_{0}}$ is a semi-coherent (see Definition 2.2.1) subregime of that $\mathbf{S}$. Then if $\mathcal{F} \neq\left\{Q_{0}\right\}, \Omega_{\mathcal{F}, Q_{0}}$ splits into two disjoint NTA domains with ADR boundary, by Lemma 2.3.12.

We may clearly assume that $a<M_{0}$. Set $\eta:=1-\frac{M_{0}+b}{M_{0}+2 b}$ and $A:=Q \backslash\left(\cup_{\mathcal{F}} Q_{j}\right)$ and $G:=\left(\cup_{\mathcal{F}} Q_{j}\right) \backslash B$. Then by (2.3.19), we see immediately that

$$
\sigma(A \cup G) \geq \eta \sigma\left(Q_{0}\right)
$$

If $\sigma(A)>(\eta / 2) \sigma\left(Q_{0}\right)$, we set $\widetilde{\Omega}=\Omega_{\mathcal{F}, Q_{0}}$, and note that $A \subseteq \partial \widetilde{\Omega}$. Then $H(a+b)$ holds in this case, by Remark 2.3.20.

Therefore it is enough to consider the case when $\sigma(G) \geq(\eta / 2) \sigma\left(Q_{0}\right)$. Suppose first that $\mathcal{F}=\left\{Q_{0}\right\}$. Then necessarily, $Q_{0} \in \mathcal{F}_{\text {good }}$, and in this case we may apply the induction hypothesis to a child of $Q_{0}$ to see that $H(a+b)$ holds. Thus, we may assume that the collection $\mathcal{F} \neq\left\{Q_{0}\right\}$. We now apply Lemma 2.3 .13 to $\mathcal{F}_{\text {good }}$ with $\kappa \geq \Upsilon$, where $\kappa$ is to be chosen momentarily, and where $\Upsilon$ is the constant in Lemma 2.2.28, to obtain a finite subcollection $\widetilde{\mathcal{F}} \subset \mathcal{F}_{\text {good }}$ with the following properties:

$$
\begin{aligned}
\sigma\left(\cup_{\widetilde{\mathcal{F}}} Q_{i}\right) & \gtrsim \sigma\left(\cup_{\mathcal{F}_{\text {good }}} Q_{j}\right) \geq(\eta / 2) \sigma\left(Q_{0}\right) \\
\operatorname{dist}\left(Q_{i}, Q_{k}\right) & \geq \kappa \max \left\{\ell\left(Q_{i}\right), \ell\left(Q_{k}\right)\right\}, \quad \forall Q_{i}, Q_{k} \in \widetilde{\mathcal{F}}, \quad i \neq k .
\end{aligned}
$$

Let $N \in \mathbb{N}$ be the cardinality of $\widetilde{\mathcal{F}}$. Now for each $Q_{j} \in \widetilde{\mathcal{F}}$, we define two families as follows: let $Q_{j}^{*}$ be the parent of $Q_{j}$, and let $Q_{j}^{\prime}$ be the cube $C_{2}$ generations down to which we can apply the induction hypothesis. Now set $\mathcal{F}^{\prime}:=\left\{Q_{j}^{\prime}\right\}_{Q_{j} \in \tilde{\mathcal{F}}}$, and 
$\mathcal{F}^{*}:=\left\{Q_{j}^{*}\right\}_{Q_{j} \in \tilde{\mathcal{F}}}$. Notice first that all of the cubes $Q_{j}^{*}$ are in $\mathbb{D}_{\mathcal{F}, Q_{0}}$ and that $\mathcal{F}^{\prime}$ has the same properties as $\widetilde{\mathcal{F}}$ namely

$$
\sigma\left(\cup_{\mathcal{F}^{\prime}} Q_{i}^{\prime}\right) \gtrsim \sigma\left(\cup_{\mathcal{F}_{\text {good }}} Q_{j}\right) \gtrsim(\eta / 2) \sigma\left(Q_{0}\right)
$$

and

$$
\operatorname{dist}\left(Q_{i}^{\prime}, Q_{k}^{\prime}\right) \geq \kappa \max \left\{\ell\left(Q_{i}^{\prime}\right), \ell\left(Q_{k}^{\prime}\right)\right\}, \quad \forall Q_{i}^{\prime}, Q_{k}^{\prime} \in \mathcal{F}^{\prime}, \quad i \neq k
$$

For each $Q_{j}^{\prime} \in \mathcal{F}^{\prime}$ we apply the induction hypothesis to obtain an open set as in $H(a)$ and call this set $\widetilde{\Omega}_{j}$.

Next, we construct two "large" NTA domains with ADR boundary with the help of Lemma 3.24 in $[\mathrm{HMM}]$. For each $Q_{j}^{\prime} \in \mathcal{F}^{\prime}$, let $\mathcal{I}_{j}$ be the collection of fattened Whitney cubes $I^{*}$ such that $\operatorname{int}\left(I^{*}\right) \subset \Omega_{\mathcal{F}, Q_{0}}$, and $I^{*}$ meets $V\left(Q_{j}^{\prime}\right)$. Let $\mathcal{B}_{j}$ be the collection of $Q \in \mathbb{D}_{Q_{0}}$ such that there exists an $I^{*} \subset U_{Q}$ with $I^{*} \in \mathcal{I}_{j}$. Now we define $\mathcal{F}_{\infty}$ as the cubes in $\mathcal{F} \cup\left(\cup_{j} \mathcal{B}_{j}\right)$ which are maximal with respect to containment.

By construction (see Remark 2.3.20), $\mathbf{S}^{\prime}:=\mathbb{D}_{\mathcal{F}_{\infty}, Q_{0}}$ is a semi-coherent subregime of some stopping time regime $\mathbf{S}$ as in Lemma 2.2.2. Thus, setting $\widetilde{\Omega}_{0}=\Omega_{F_{\infty}, Q_{0}}$, by Lemma 2.3.12 we obtain that $\Omega_{0}$ is the union of two disjoint NTA domains with ADR boundaries, whose diameters are comparable to $\ell\left(Q_{0}\right)$.

We will need to know that we did not remove too many cubes, namely, we do not want to remove any $Q_{j}^{*} \in \mathcal{F}^{*}$.

Claim 2.3.23. If $\kappa$ is chosen large enough then for every $j \geq 1$ we have for each $Q_{j}^{*} \in \mathcal{F}^{*}$ that $Q_{j}^{*} \in \mathbb{D}_{\mathcal{F}_{\infty}, Q_{0}}$.

Proof of claim: Note that by our choice of $C_{2}, U_{Q}$ does not meet $V\left(Q_{j}^{\prime}\right)$, for any $Q \supseteq Q_{j}^{*}$. Moreover, by construction, we have that $Q_{j}^{*} \notin \mathcal{F}$. Suppose now for the 
purposes of contradiction, that there is a $j \geq 1$ such that $Q_{j}^{*} \subseteq Q$, with $Q \in \mathcal{F}_{\infty}$. Then there exists a $k \neq j$ such that $Q \in \mathcal{B}_{k}$ and $U_{Q}$ meets $V\left(Q_{k}^{\prime}\right)$ for some $Q_{k}^{\prime} \in \mathcal{F}^{\prime}$. Then we have immediately that $\ell(Q) \lesssim \ell\left(Q_{k}^{\prime}\right)<\ell\left(Q_{k}\right)$ and $\operatorname{dist}\left(Q_{k}^{\prime}, Q\right) \lesssim \ell\left(Q_{k}^{\prime}\right)<\ell\left(Q_{k}\right)$ so that

$$
\operatorname{dist}\left(Q_{k}^{\prime}, Q_{j}^{\prime}\right) \lesssim \operatorname{dist}\left(Q_{k}^{\prime}, Q\right)+\ell(Q) \lesssim \ell\left(Q_{k}\right)
$$

a contradiction for $\kappa$ large enough. We remind the reader that we are only considering the cubes extracted with a covering lemma so that they separated (see Lemma 2.3.13 and (2.3.22)).

Remark 2.3.24. We note for future reference that $V\left(Q_{j}^{\prime}\right) \cap\left(\widetilde{\Omega}_{0} \cup \partial \widetilde{\Omega}_{0}\right)=\varnothing$, for every $j \geq 1$. Indeed, this follows by our observation, in the preceding paragraph, that by choice of $C_{2}$ large enough, $U_{Q}$ does not meet $V\left(Q_{j}^{\prime}\right)$, for any $Q \supseteq Q_{j}^{*}$. We further note that $\overline{V\left(Q_{j}^{\prime}\right)} \cap \overline{V\left(Q_{k}^{\prime}\right)}=\varnothing$, for all $j \neq k$, with $j, k \geq 1$, by (2.3.22), Lemma 2.2.28, and our choice of $\kappa \geq \Upsilon$.

Recall that for $1 \leq j \leq N, \widetilde{\Omega}_{j}$ is the open set associated to $Q_{j}^{\prime} \in \mathcal{F}^{\prime}$ via the induction hypothesis, and $\widetilde{\Omega}_{0}=\Omega_{F_{\infty}, Q_{0}}$. We now set $\widetilde{\Omega}:=\cup_{j=0}^{N} \widetilde{\Omega}_{j}$. Note that $\widetilde{\Omega}$ has only finitely many components by hypothesis. We shall show that $\widetilde{\Omega}$ has all the desired properties. First we show that $\widetilde{\Omega}$ satisfies a 2-sided Corkscrew condition. Note that by Remark 2.3.24, the distinct components of $\widetilde{\Omega}_{j}$ remain distinct components in $\widetilde{\Omega}$. We let $x \in \partial \widetilde{\Omega}$ and $0<r \leq \ell\left(Q_{0}\right)$, let $M$ be a large number to be chosen, and set $\delta(x)=\operatorname{dist}(x, E)$. Since $\partial \widetilde{\Omega} \subseteq \cup_{j=0}^{N} \partial \widetilde{\Omega}_{j}$ we break into two cases:

Case 1: $x \in \partial \widetilde{\Omega}_{0}$. Recall that $\widetilde{\Omega}_{0}$ splits into two NTA domains $\widetilde{\Omega}_{0}^{ \pm}$. Moreover, following the construction in $[\mathrm{HMM}]$, the interior and exterior corkscrew points for the domain $\widetilde{\Omega}_{0}^{+}$are found as follows. Without loss of generality we may assume 
that $x \in \partial \widetilde{\Omega}_{0}^{+}$. For M sufficiently large (depending only on allowable constants) the argument distinguishes between two cases, when $r<M \delta(x)$ and when $r \geq M \delta(x)$. In the case that $r \geq M \delta(x)$ we find one Corkscrew point in the domain $\widetilde{\Omega}_{0}^{-}$and one Corkscrew point in $\widetilde{\Omega}_{0}^{+}$, and these serve as Corkscrew points in separate components for $\widetilde{\Omega}$ as well. In the case that $r<M \delta(x)$, we have that $\delta(x)>0$, and $x$ lies on the face of a fattened Whitney cube $I^{*}$ whose interior lies in $\widetilde{\Omega}_{0}^{+}$. Moreover, $x \in J$, for some Whitney cube $J \notin\left(\cup_{Q \in \mathbb{D}_{\mathcal{F}_{\infty}, Q_{0}}} \mathcal{W}_{Q}\right)$; we then have one Corkscrew point in $I^{*}$, and a second in $J \backslash \widetilde{\Omega}_{0}^{+}$. Clearly, the first of these is also a Corkscrew point for $\widetilde{\Omega}$, in the component $\widetilde{\Omega}_{0}^{+}$. To see that the second is a Corkscrew point relative to $\partial \widetilde{\Omega}$, it remains to show that $J$ misses $\partial \widetilde{\Omega} \backslash \partial \widetilde{\Omega}_{0}^{+}$. If not, then $J$ must intersect $\partial \widetilde{\Omega}_{j}$ for some $1 \leq j \leq N$, and therefore $T_{Q_{j}^{\prime}}$ meets $J$, so that $J \subset V\left(Q_{j}^{\prime}\right)$. On the other hand, $J$ also meets $I^{*}$, so that $I^{*} \in \mathcal{I}_{j}$, hence there is a cube $Q \in \mathbb{D}_{\mathcal{F}_{\infty}, Q_{0}}$ that belongs to $\mathcal{B}_{j}$, a contradiction.

Case 2: $x \in \partial \widetilde{\Omega}_{j}$ for $1 \leq j \leq N$. Here $\widetilde{\Omega}_{j}$ is associated to $Q_{j}^{\prime}$.

Case 2a: $r \geq M \ell\left(Q_{j}^{\prime}\right)$. In this case, since $Q_{j}^{*} \in \mathbb{D}_{\mathcal{F}_{\infty}, Q_{0}}$, we have that $\operatorname{int}\left(U_{Q_{j}^{*}}^{ \pm}\right) \subset \widetilde{\Omega}_{0}^{ \pm}$, so for $M$ large enough depending on $C_{2}$, there exists points $y^{ \pm} \in \partial \widetilde{\Omega}_{0}^{ \pm}$, with $\left|x-y^{ \pm}\right|<$ $r / 2$. We may then apply Case 1 to each of the balls $B\left(y^{ \pm}, r / 2\right) \subset B(x, r)$.

Case 2b: $r<M \ell\left(Q_{j}^{\prime}\right)$. From the induction hypothesis we have two Corkscrew balls $B_{1}$ and $B_{2}$ that satisfy the strong 2 -sided Corkscrew condition, at scale $r / M$, relative to the open set $\widetilde{\Omega}_{j}$ (see Definition 2.1.9). Without loss of generality we may assume that $B_{1} \subset \widetilde{\Omega}_{j}$, hence also $B_{1} \subset \widetilde{\Omega}$. We must show that these Corkscrew balls satisfy the strong 2 -sided Corkscrew condition for open sets, with $\widetilde{\Omega}$ the open set in question, thus, it remains to show that $B_{1}, B_{2} \subset \mathbb{R}^{n+1} \backslash \partial \widetilde{\Omega}$. To this end, we simply observe 
that $B_{1}$ and $B_{2}$ do not meet $\partial \widetilde{\Omega}_{j}$ by hypothesis, nor they do not meet $\partial \widetilde{\Omega}_{0}$ or $\partial \widetilde{\Omega}_{i}$ for some $i \neq j$, by Remark 2.3.24, since by the induction hypothesis $B_{1}, B_{2}$ are in $V\left(Q_{j}^{\prime}\right)$, and since $\partial \widetilde{\Omega}_{i} \subset \overline{V\left(Q_{i}^{\prime}\right)}$, by construction.

Next, we observe that $\partial \widetilde{\Omega}_{0}$ is ADR, by Lemma 2.3.12, and that each $\partial \widetilde{\Omega}_{j}$ is ADR, with uniform control of the ADR constants, by the induction hypothesis. Thus, we are left with showing that $\widetilde{\Omega}$ has ADR boundary and that condition 2.3.17 holds. We begin by verifying the upper ADR condition for $\partial \widetilde{\Omega}$ with bound independent of $N$. Let $x \in \partial \widetilde{\Omega}$ and $0<r \leq \operatorname{diam}\left(Q_{0}\right)$. Since $\partial \widetilde{\Omega} \subseteq \cup_{j=0}^{N} \partial \widetilde{\Omega}_{j}$,

$$
H^{n}(B(x, r) \cap \partial \widetilde{\Omega}) \leq \sum_{j=0}^{N} H^{n}\left(B(x, r) \cap \partial \widetilde{\Omega}_{j}\right)
$$

If $B(x, r)$ meets $\partial \widetilde{\Omega}_{0}$, then there is an $x_{0} \in B(x, r) \cap \partial \widetilde{\Omega}_{0}$, and $B\left(x_{0}, 2 r\right) \supset B(x, r)$. By the ADR property for $\widetilde{\Omega}_{0}$

$$
H^{n}\left(B(x, r) \cap \partial \widetilde{\Omega}_{0}\right) \leq H^{n}\left(B\left(x_{0}, 2 r\right) \cap \partial \widetilde{\Omega}_{0}\right) \lesssim r^{n} .
$$

Next, we consider the contributions of $\partial \widetilde{\Omega}_{j}, 1 \leq j \leq N$, which we write as

$$
\sum_{j=1}^{N} H^{n}\left(B(x, r) \cap \partial \widetilde{\Omega}_{j}\right)=\sum_{j: \ell\left(Q_{j}^{\prime}\right)>r}+\sum_{j: \ell\left(Q_{j}^{\prime}\right) \leq r}=: I+I I .
$$

We recall that by construction $\partial \widetilde{\Omega}_{j} \subset \overline{V\left(Q_{j}^{\prime}\right)}$, so by Remark 2.3 .24 , the boundaries $\partial \widetilde{\Omega}_{j}$ are pairwise disjoint. Thus, only a bounded number of terms (with bound independent of $N$ ) can appear in the sum $I$, so the desired bound $I \lesssim r^{n}$ follows by the ADR property of each $\partial \widetilde{\Omega}_{j}$. Moreover, for each $j$, the diameter of $\widetilde{\Omega}_{j}$ is comparable to $\ell\left(Q_{j}^{\prime}\right)$, and therefore the cubes $Q_{j}^{\prime}$ appearing in $I I$ are all contained in $B(x, C r)$, for some sufficiently large constant $C$ depending only on allowable parameters. Consequently, 
by the ADR property of $\partial \widetilde{\Omega}_{j}$ and of $E$,

$$
I I \lesssim \sum_{j: Q_{j}^{\prime} \subset B(x, C r)} H^{n}\left(\partial \widetilde{\Omega}_{j}\right) \approx \sum_{j: Q_{j}^{\prime} \subset B(x, C r)} \sigma\left(Q_{j}^{\prime}\right) \leq \sigma(E \cap B(x, C r)) \approx r^{n} .
$$

Thus, we obtain the upper ADR bound (independent on $N$ ).

With the upper ADR bound in hand we now know that $\partial \widetilde{\Omega}$ is a set of locally finite perimeter, so by the isoperimetric inequality [EG, p. 222], and the strong two sided Corkscrew condition for open sets, the lower ADR bound follows. The last and easiest thing to show is that condition (2.3.17) holds for $\widetilde{\Omega}$, but this follows readily from 2.3.21, and the fact that by the induction hypothesis, 2.3.17 hold for each $Q_{j}^{\prime}$.

\subsection{Theorems 2.4.1 and 2.4.9: Applications to Har- monic Measure}

We now explore how Theorem 2.3.1 relates to harmonic measure.

Theorem 2.4.1. Let $E \subset \mathbb{R}^{n+1}$ be an $n$-dimensional UR set. Let $\Omega:=\mathbb{R}^{n+1} \backslash E$. Then E has "interior big pieces of good harmonic measure estimates" (IBP(GHME)), that is, we have the following: for each $Q \in \mathbb{D}(E)$ there exists an open set $\widetilde{\Omega}=\widetilde{\Omega}_{Q} \subset \Omega$, with $\operatorname{diam}(\widetilde{\Omega}) \approx \operatorname{diam}(Q)$, such that $\widetilde{\Omega}$ satisfies a strong 2-sided corkscrew condition along with estimate (2.3.2), and for each surface ball $\Delta=\Delta(x, r):=B(x, r) \cap \partial \widetilde{\Omega}$, with $x \in \partial \widetilde{\Omega}$ and $r \in(0, \operatorname{diam}(\widetilde{\Omega}))$, and with interior corkscrew point $X_{\Delta}$, it holds that $\omega^{X_{\Delta}}:=\omega_{\widetilde{\Omega}}^{X_{\Delta}}$, the harmonic measure for $\widetilde{\Omega}$ with pole at $X_{\Delta}$, belongs to weak- $A_{\infty}(\Delta)$. (Here the open set $\widetilde{\Omega} \subset \Omega$ is the one that we constructed in Theorem 2.3.1.)

Thus, every UR set of co-dimension 1 has big pieces of sets satisfying a quantitative, scale invariant F. and M. Riesz Theorem. We remark that this fact actually characterizes uniformly rectifiable sets of co-dimension 1 (see [HM3]). 
Corollary 2.4.2. Let $E \subset \mathbb{R}^{n+1}$ be an $A D R$ set. Then $E$ is $U R$ if and only if $E$ has $\operatorname{IBP}(G H M E)$.

The proof of Theorem 2.4.1 is an immediate consequence of the following Lemma and Theorem 2.3.1.

Lemma 2.4.3. Let $\Omega$ be a bounded open set in $\mathbb{R}^{n+1}$, with $n$-dimensional ADR boundary, such that $\Omega:=\cup_{j} \Omega_{j}$ is the union of its connected components $\Omega_{j}$. Suppose further that each component is a chord arc domain with uniform bounds on the chord arc constants. For $x_{0} \in \partial \Omega$ and $r_{0}<\frac{1}{2} \operatorname{diam} \Omega$, and for $Y \in \Omega \backslash B\left(x_{0}, 2 r_{0}\right)$, let $\omega^{Y}$ denote the harmonic measure associated to $\Omega$, and set $\Delta_{0}:=B\left(x_{0}, r_{0}\right) \cap \partial \Omega$. Then $\omega^{Y} \in$ weak- $A_{\infty}\left(\Delta_{0}\right)$, with uniform control on the weak- $A_{\infty}$ constants.

Proof. Fix $B_{0}:=B\left(x_{0}, r_{0}\right)$, with $x_{0} \in \partial \Omega$, and $r_{0}<\operatorname{diam}(\partial \Omega)$. Set $\Delta=\Delta(x, r)$ and $2 \Delta=\Delta(x, 2 r)$, with $x \in \partial \Omega$, and suppose that $B(x, 2 r) \subset B_{0}$ (thus, $r_{0} \geq 2 r$ ). Recalling the definition of weak- $A_{\infty}$, we need to show there exist uniform positive constants $C$ and $\theta$ such that for each Borel set $A \subseteq \Delta$

$$
\omega^{Y}(A) \leq C\left(\frac{\sigma(A)}{\sigma(\Delta)}\right)^{\theta} \omega(2 \Delta)
$$

whenever $Y \in \Omega \backslash B\left(x_{0}, 2 r_{0}\right)$. Let us fix such a point $Y$. We note that $Y \in \Omega_{j}$ for some $j$ and therefore $\omega^{Y}$ is just the harmonic measure associated to the domain $\Omega_{j}$. Thus, if $A \cap \partial \Omega_{j}=\varnothing$, then (2.4.4) holds trivially. We may therefore assume that this is not the case. Let $z \in A \cap \partial \Omega_{j}$, set $A^{\prime}=A \cap \partial \Omega_{j}$, and set $\sigma_{j}=\left.H^{n}\right|_{\partial \Omega_{j}}$. Notice that $\operatorname{dist}(z, Y) \geq r_{0} \geq 2 r$, since in particular, $z \in B_{0}$, while $Y \in \Omega \backslash 2 B_{0}$. Thus, the diameter of $\Omega_{j}$ must be greater than $r$. Moreover, by the result obtained independently in [DJ] and in [Se], $\omega^{Y} \in A_{\infty}\left(2 \Delta_{\star}\right)$, where $\Delta_{\star}=B(z, r) \cap \Omega_{j}, 2 \Delta_{\star}=$ 
$B(z, 2 r) \cap \Omega_{j}$, and in particular, we have the doubling estimate

$$
\omega^{Y}\left(2 \Delta_{\star}\right) \lesssim \omega^{Y}\left(\Delta_{\star}\right)
$$

Note further that $\Delta_{\star} \subset 2 \Delta$, that $A^{\prime} \subset 2 \Delta_{\star}$, and also that by the uniform ADR property, $\sigma(\Delta) \approx \sigma_{j}\left(\Delta_{\star}\right)=\sigma\left(\Delta_{\star}\right)$.

Since $\omega^{Y} \in A_{\infty}\left(2 \Delta_{\star}\right)$, we therefore have that

$$
\begin{aligned}
\omega^{Y}(A) & =\omega^{Y}\left(A^{\prime}\right) \\
& \leq C\left(\frac{\sigma_{j}\left(A^{\prime}\right)}{\sigma_{j}\left(\Delta_{\star}\right)}\right)^{\theta} \omega^{Y}\left(2 \Delta_{\star}\right) \\
& \leq C\left(\frac{\sigma\left(A^{\prime}\right)}{\sigma\left(\Delta_{\star}\right)}\right)^{\theta} \omega^{Y}\left(\Delta_{\star}\right) \lesssim\left(\frac{\sigma(A)}{\sigma(\Delta)}\right)^{\theta} \omega^{Y}(2 \Delta),
\end{aligned}
$$

where in the next to last inequality, we have used (2.4.5).

To prove Theorem 2.4.1, we apply the preceding lemma to each domain $\widetilde{\Omega}$ constructed in Theorem 2.3.1. We need only verify that the point $Y \in \widetilde{\Omega} \backslash 2 B_{0}$ may be replaced by any Corkscrew point $X_{\Delta_{0}} \in \widetilde{\Omega}$, relative to the surface ball $\Delta_{0}$. To this end, we fix such a Corkscrew point $X_{\Delta_{0}}$, and observe that $X_{\Delta_{0}} \in \widetilde{\Omega} \backslash 2 B_{1}$, for any ball $B_{1}$ meeting $B_{0}$, centered on $\partial \widetilde{\Omega}$, with radius $r_{1}=\eta r_{0}$, if $\eta>0$ is chosen small enough depending only on the Corkscrew constant for $\widetilde{\Omega}$. Then for each such $B_{1}$, by Lemma 2.4.3, $\omega_{0}:=\omega^{X_{\Delta_{0}}}$ belongs to weak- $A_{\infty}\left(\Delta_{1}\right)$, where $\Delta_{1}=B_{1} \cap \partial \widetilde{\Omega}$. Now suppose that $B(x, 2 r) \subset B_{0}$, with $x \in \partial \widetilde{\Omega}$, and observe that $B:=B(x, r)$ may be covered by a collection of balls $\mathcal{F}=\left\{B^{i}:=B\left(x^{i}, r^{i}\right)\right\}$, of bounded cardinality depending only on dimension and $\eta$, such that $r^{i} \approx r, 2 B^{i} \subset 2 B$, and each $B^{i}$ is contained in some ball $B_{1}=B_{1}^{i}$ as above. Set $\Delta=B \cap \partial \widetilde{\Omega}, \Delta^{i}:=B^{i} \cap \partial \widetilde{\Omega}$, and for $A \subset \Delta$, let $A_{i}:=A \cap \Delta^{i}$. We then apply (2.4.4) to each $A_{i}, \Delta^{i}$, and use that for each $i, H^{n}(\Delta) \approx H^{n}\left(\Delta^{i}\right)$ 
(depending on $\eta$ and the ADR constants). Since $\# \mathcal{F}$ is bounded, we may then sum in $i$ to obtain Theorem 2.4.1.

We conclude by observing that Lemma 2.4.3, and hence Theorem 2.3.1, also apply to the Riesz measure ("p-harmonic measure") associated to the $p$-Laplace equation

$$
\Delta_{p} u:=\operatorname{div}\left(|\nabla u|^{p-2} \nabla u\right)=0
$$

Definition 2.4.6. ( $p$-harmonic measure). Let $\Omega \subset \mathbb{R}^{n+1}$ be open. For $x \in \partial \Omega$, $0<r<\frac{1}{8} \operatorname{diam}(\Omega)$, suppose that $u \geq 0$, with $u \equiv 0$ on $\partial \Omega, \Delta_{p} u=0$ in $4 B \cap \Omega$. We define the $p$-harmonic measure $\mu$ associated to $u$, as the unique finite positive Borel measure such that

$$
-\iint_{\Omega}|\nabla u|^{p-2} \nabla u \cdot \nabla \Phi d x=\int_{\partial \Omega} \Phi d \mu \quad \forall \Phi \in C_{0}^{\infty}(4 B) .
$$

Proposition 2.4.8. Let $\Omega$ be a bounded open set in $\mathbb{R}^{n+1}$, with $n$-dimensional $A D R$ boundary, such that $\Omega:=\cup_{j} \Omega_{j}$ is the union of its connected components $\Omega_{j}$, and satisfies a strong 2-sided corkscrew condition. Suppose further that each component $\Omega_{j}$ is a chord arc domain with uniform bounds on the chord arc constants. Then for $x \in \partial \Omega$ and $r<\frac{1}{8} \operatorname{diam}(\Omega)$, let $u, \mu$ be as above, set $\hat{\mu}:=\left.\mu\right|_{\partial \Omega_{j}}$ and let $\hat{c}$ be the corkscrew constant for the set $\Omega$. If $\operatorname{diam}\left(\Omega_{j}\right)>2 \hat{c} r$, then $\hat{\mu} \in$ weak- $A_{\infty}(\Delta)$. In particular, given $x \in \partial \Omega$ and $r \in\left(0, \frac{1}{8} \operatorname{diam}(\Omega)\right)$, if we let $\Omega_{\Delta}$ be some component which contains an interior corkscrew point relative to the ball $B(x, r)$, then $\left.\mu\right|_{\partial \Omega_{\Delta}} \in$ weak- $A_{\infty}(\Delta)$

Proof. The proof is the same as that of Lemma 2.4.3. Lewis and Nyström [LN] showed that for an NTA domain $D$ with ADR boundary, if $\mu$ is $p$-harmonic measure associated to a non-trivial positive $p$-harmonic function $u$ in $B(x, 4 r) \cap D$, with $x \in$ 
$\partial D, r<\operatorname{diam}(D) / 4$, then $\mu \in A_{\infty}(\partial D \cap B(x, r))$, with respect to $\sigma_{D}:=\left.H^{n}\right|_{\partial D}$; we use this fact in place of the result of $[\mathrm{DJ}]$, [Se].

By modifying Theorem 2.3.1 we are able to show that $\sigma \ll \omega$ under certain background hypothesis, that is, we show the following.

Theorem 2.4.9. If $\Omega \subset \mathbb{R}^{n+1}, n \geq 1$, is a connected domain satisfying an interior corkscrew condition and $\partial \Omega$ is $U R$ then $\sigma \ll \omega$.

First, we state and prove another structure result that will allow us to prove Theorem 2.4.9.

Proposition 2.4.10. Suppose $\Omega$ is a connected open set satisfying an interior corkscrew condition and that $\partial \Omega$ is UR. Then there exists $\eta>0$ such that for each $Q_{0}$ in $\partial \Omega$ and a countable collection of disjoint subcubes of $Q_{0},\left\{Q_{j}\right\}$, with the property for that for each $Q_{j}$ there exists a family of subcubes $\mathcal{F}_{j}$ such that $\Omega_{\mathcal{F}_{j}, Q_{j}}^{*}$ is an NTA domain (with uniform control on the NTA constants) such that $\Omega_{\mathcal{F}_{j}, Q_{j}}^{*} \subset \Omega$ and that

$$
\sigma\left(Q_{0} \cap\left(\cup_{j} \partial \Omega_{\mathcal{F}_{j}, Q_{j}}^{*}\right) \geq \eta \sigma\left(Q_{0}\right)\right.
$$

Proof. Let $\partial \Omega=E$. First we fix $\eta$ and $K$ so that that Lemma 2.3.12 holds and let $\mathfrak{m}$ be the packing measure for the bilateral corona decomposition (as in the proof of Theorem 2.3.1). By the hypothesis that $\Omega$ satisfies the interior corkscrew condition there exists a constant $c$ such that for $Q$ in $\mathbb{D}(E)=\mathbb{D}(\partial \Omega)$ if $x_{Q}$ is the 'center' of the cube $Q$, then for $r \approx \ell(Q)<\operatorname{diam}(\partial \Omega)$ there is a corkscrew ball $B_{Q}=B\left(X_{Q}, c r\right) \subset B\left(x_{Q}, r\right) \cap \Omega$. By adjusting $\eta$ and $K$ we may ensure that $B_{Q} \cap \mathcal{W}_{Q}^{0} \neq \varnothing$, thus when constructing $\Omega_{\mathbf{S}^{\prime}}^{ \pm}$as in Lemma 2.3.12 one of the NTA domains may be taken to be interior to $\Omega$, we call this domain (pick one if both are 
interior) $\Omega_{\mathbf{S}^{\prime}}^{*}$. Now the proof will follow in the same way as Theorem 2.3.1. For any $a \geq 0$ we have the induction hypothesis $H(a)$, defined in the following way.

$H(a)$ : There exists $\eta_{a}>0$ such that for all $Q_{0} \in \mathbb{D}(E)$ satisfying $\mathfrak{m}\left(\mathbb{D}_{Q_{0}}\right) \leq a \sigma\left(Q_{0}\right)$, there exists a $\eta_{a}>0$ and a countable collection of disjoint subcubes of $Q_{0},\left\{Q_{j}\right\}$, with the property for that for each $Q_{j}$ there exists a family of subcubes $\mathcal{F}_{j}$ such that $\Omega_{\mathcal{F}_{j}, Q_{j}}^{*}$ is an NTA domain (with uniform control on the NTA constants) and

$$
\sigma\left(Q_{0} \cap\left(\cup_{j} \partial \Omega_{\mathcal{F}_{j}, Q_{j}}^{*}\right) \geq \eta_{a} \sigma\left(Q_{0}\right)\right.
$$

To prove the Proposition it is enough to show that $H\left(M_{0}\right)$ holds where $M_{0}$ is the Carleson norm of $\mathfrak{m}$, that is, $M_{0}=C_{\eta, K}$ in Lemma 2.2.2. We do this by showing first that $H(0)$ holds and then that $H(a)$ implies $H(a+b)$ for some fixed constant $b$ depending only on dimension and the ADR constants. This way we will only use finitely many steps to get to $H\left(M_{0}\right)$. We set $b=\frac{\gamma}{C}$ where $C$ is the constant in (2.3.10) and $\gamma$ is a small positive number to be chosen.

Again, the fact that $H(0)$ holds is somewhat trivial since this would imply that $Q_{0}$ and all of its descendants are in the same stopping time regime and therefore $Q_{0}$ lies on the boundary of a NTA domain with ADR boundary.

Proof that $H(a) \Longrightarrow H(a+b)$ : Suppose that $H(a)$ holds. Let $Q_{0} \in \mathbb{D}(E)$ be such that $\mathfrak{m}\left(\mathbb{D}_{Q_{0}}\right) \leq(a+b) \sigma\left(Q_{0}\right)$. We obtain via Lemma 2.3 .9 a collection $\mathcal{F}=\left\{Q_{j}\right\}$ such that

$$
\left\|\mathfrak{m}_{\mathcal{F}}\right\|_{C\left(Q_{0}\right)} \leq C b=\gamma
$$

and

$$
\sigma(B) \leq \frac{a+b}{a+2 b} \sigma\left(Q_{0}\right)
$$


where $B$ is the union of those $Q_{j}$ in $\mathcal{F}$ such that $\mathfrak{m}\left(\mathbb{D}_{Q_{j}} \backslash\left\{Q_{j}\right\}\right)>a \sigma\left(Q_{j}\right)$, call this collection $\mathcal{F}_{\text {bad }}$. Define $\mathcal{F}_{\text {good }}:=\mathcal{F} \backslash \mathcal{F}_{\text {bad }}$. Then by pigeon-holing, for each $Q_{j} \in \mathcal{F}_{\text {good }}$ we may find a child of $Q_{j}$ to which we can apply the induction hypothesis $H(a)$.

Remark 2.4.15. Again, choosing $\gamma$ small enough in (2.4.13) (in fact, $\gamma=1 / 2$ will suffice), we obtain that $\mathbb{D}_{\mathcal{F}, Q_{0}}$ does not contain any $Q^{\prime} \in \mathcal{M} \cup \mathcal{B}$, where $\mathcal{M}:=\{Q(\mathbf{S})\}_{\mathbf{S}}$. Fixing such a $\gamma$, we find therefore that every $Q^{\prime} \in \mathbb{D}_{\mathcal{F}, Q_{0}}$ is a good cube, and moreover all such $Q^{\prime}$ belong to the same $\mathbf{S}$, a stopping time regime as in Lemma 2.2.2. Thus, $\mathbb{D}_{\mathcal{F}, Q_{0}}$ is a semi-coherent subregime of that $\mathbf{S}$. Then if $\mathcal{F} \neq\left\{Q_{0}\right\}, \Omega_{\mathcal{F}, Q_{0}}$ splits into two disjoint NTA domains with ADR boundary, by Lemma 2.3.12. If this is the case $\left(\mathcal{F} \neq\left\{Q_{0}\right\}\right)$, we choose an interior domain $\Omega_{\mathcal{F}, Q_{0}}^{*}$.

We may clearly assume that $a<M_{0}$. Set $\eta:=1-\frac{M_{0}+b}{M_{0}+2 b}$ and $A:=Q \backslash\left(\cup_{\mathcal{F}} Q_{j}\right)$ and $G:=\left(\cup_{\mathcal{F}} Q_{j}\right) \backslash B$. Then by (2.4.14), we see immediately that

$$
\sigma(A \cup G) \geq \eta \sigma\left(Q_{0}\right)
$$

If $\sigma(A)>(\eta / 2) \sigma\left(Q_{0}\right)$, we take for our collection $\left\{Q_{0}\right\}$ with the single family $\mathcal{F}$. Then $H(a+b)$ holds in this case, by Remark 2.4.15.

Therefore it is enough to consider the case when $\sigma(G) \geq(\eta / 2) \sigma\left(Q_{0}\right)$. Suppose first that $\mathcal{F}=\left\{Q_{0}\right\}$. Then necessarily, $Q_{0} \in \mathcal{F}_{\text {good }}$, and in this case we may apply the induction hypothesis to a child of $Q_{0}$ to see that $H(a+b)$ holds. Thus, we may assume that the collection $\mathcal{F} \neq\left\{Q_{0}\right\}$. Now for each $Q_{j}^{\prime} \in \mathcal{F}_{\text {good }}$ we may apply the induction hypothesis to $Q_{j}^{\prime}$ to obtain the existence of a collection of subcubes of $Q_{j}^{\prime}$, $\left\{Q_{j}^{k}\right\}$, which are disjoint and for each $Q_{j}^{k}$ there exists a disjoint collection of subcubes $\mathcal{F}_{j}^{k}$, such that $\Omega_{\mathcal{F}_{j}^{k}, Q_{j}^{k}}$ is NTA and 


$$
\sigma\left(Q_{j} \cap\left(\cup_{k} \partial \Omega_{\mathcal{F}_{j}^{k}, Q_{j}^{k}}^{*}\right)\right) \geq \eta_{a} \sigma\left(Q_{j}\right)
$$

This implies that

$$
\sigma\left(Q_{0} \bigcap\left(\bigcup_{j, k} \partial \Omega_{\mathcal{F}_{j}^{k}, Q_{j}^{k}}^{*}\right)\right) \geq \eta_{a}(\eta / 2) \sigma\left(Q_{0}\right)=\eta_{a+b} \sigma\left(Q_{0}\right),
$$

which after reindexing $\left\{Q_{j}^{k}\right\}$ proves the Proposition.

Now we are ready to prove Theorem 2.4.9.

Proof of Theorem 2.4.9. Suppose that $F \subset \partial \Omega$ is such that $\sigma(F)>0$, then there exists a $Q_{0} \in \mathbb{D}(\partial \Omega)$ such that $\sigma\left(F \cap Q_{0}\right)=\kappa>0$. Since $\partial \Omega$ is UR we apply Proposition 2.4.10 to $Q_{0}$ to obtain a collection of cubes $\left\{Q_{j}\right\}$ and associated $\mathcal{F}_{j}$ such that

$$
\sigma\left(Q_{0} \cap\left(\cup_{j} \partial \Omega_{\mathcal{F}_{j}, Q_{j}}^{*}\right)\right)>\eta \sigma\left(Q_{0}\right)
$$

and each $\Omega_{\mathcal{F}_{j}, Q_{j}}^{*}$ is an NTA domain (with uniform control on the constants). By inner regularity of $H^{n}$ measure restricted to $Q_{0}$ we may approximate $Q_{0} \cap\left(\cup_{j} \partial \Omega_{\mathcal{F}_{j}, Q_{j}}^{*}\right)$ by a closed set $G \subset Q_{0} \cap\left(\cup_{j} \partial \Omega_{\mathcal{F}_{j}, Q_{j}}^{+}\right)$such that

$$
\sigma\left(G \cap Q_{0}\right) \geq(\eta / 2) \sigma\left(Q_{0}\right)
$$

Since $G \cup \partial Q_{0}$ closed we may decompose $\overline{Q_{0}} \backslash G$ (up to a set of $H^{n}$ measure zero) into a collection of disjoint cubes in $\mathbb{D}\left(Q_{0}\right)$ maximal with respect to containment, call this collection $\mathcal{F}^{1}=\left\{Q_{k}^{1}\right\}$. Notice then that

$$
\sigma\left(Q_{0} \cap\left(\cup_{k} Q_{k}^{1}\right)\right)=\sum_{k} \sigma\left(Q_{k}^{1}\right) \leq(1-(\eta / 2)) \sigma\left(Q_{0}\right)
$$

We may now apply Proposition 2.4.10 to each cube $Q_{k}^{1}$ in $\mathcal{F}^{1}$, obtaining for a collection $Q_{k, j}$ and associated family $\mathcal{F}_{k, j}$ such that

$$
\sigma\left(Q_{k}^{1} \cap\left(\cup_{j} \partial \Omega_{\mathcal{F}_{k, j}, Q_{k, j}}^{*}\right)\right)>\eta \sigma\left(Q_{k}^{1}\right)
$$


Again for each $Q_{k}^{1}$ approximate $Q_{k}^{1} \cap\left(\cup_{j} \partial \Omega_{\mathcal{F}_{k, j}, Q_{k, j}}^{+}\right)$by a closed set $G_{k}$ such that

$$
\sigma\left(Q_{k}^{1} \cap G_{k}\right) \geq(\eta / 2) \sigma\left(Q_{k}^{1}\right)
$$

Since $G_{k} \cup \partial Q_{k}^{1}$ is closed we may decompose $\overline{Q_{k}^{1}} \backslash G_{k}$ (up to a set of $H^{n}$ measure zero) into a collection of disjoint cubes in $\mathbb{D}\left(Q_{k}^{1}\right)$ maximal with respect to containment, call this collection $\mathcal{F}_{k}^{2}$. It then follows that

$$
\sigma\left(Q_{k}^{1} \cap\left(\cup_{Q \in \mathcal{F}_{k}^{2}} Q\right)\right) \leq(1-(\eta / 2)) \sigma\left(Q_{k}^{1}\right)
$$

Let $\mathcal{F}^{2}=\cup_{k} \mathcal{F}_{k}^{2}$, then it follows from (2.4.20) and (2.4.23) that

$$
\begin{aligned}
\sigma\left(Q_{0} \cap\left(\cup_{Q \in \mathcal{F}^{2}} Q\right)\right) & =\sum_{k} \sigma\left(Q_{k}^{1} \cap\left(\cup_{Q \in \mathcal{F}_{k}^{2}} Q\right)\right) \\
& \leq \sum_{k}(1-(\eta / 2)) \sigma\left(Q_{k}^{1}\right) \\
& \leq(1-(\eta / 2))^{2} \sigma\left(Q_{0}\right) .
\end{aligned}
$$

Continuing this way we may exhaust boundary. Instead, let us just just consider the following. Let $M$ be large enough so that $(1-(\eta / 2))^{M} \sigma\left(Q_{0}\right)<\kappa / 2$, then since

$$
\sigma\left(Q_{0} \cap\left(\cup_{Q \in \mathcal{F}^{M}} Q\right)\right) \leq(1-(\eta / 2))^{M} \sigma\left(Q_{0}\right)<\kappa / 2 .
$$

Then it must be the case that there exists a cube $Q \in \mathbb{D}\left(Q_{0}\right)$ and an NTA domain $\Omega_{\mathcal{F}_{Q}, Q}^{*}=\Omega^{\prime}$ such that $H^{n}\left(F \cap \partial \Omega^{\prime}\right)>0$. Let $X$ be any point in $\Omega^{\prime}$ and let $\omega_{\Omega^{\prime}}^{X}$ be the harmonic measure associated to $\Omega^{\prime}$. Since $\omega_{\Omega^{\prime}}^{X} \in A_{\infty}\left(H^{n} L \partial \Omega^{\prime}\right)$ by the work of David and Jerision in [DJ], it follows that $\omega_{\Omega^{\prime}}^{X}\left(F \cap \partial \Omega^{\prime}\right)>0$. By the maximum principle we then have that $\omega^{X}(F) \geq \omega_{\Omega^{\prime}}^{X}\left(F \cap \partial \Omega^{\prime}\right)>0$. By the Harnack inequality $\omega^{Y}(F)>0$ for any $Y \in \Omega$. 


\section{Chapter 3}

\section{Rectifiability and Harmonic Measure}

In this chapter we look at harmonic measure for open sets with rectifiable boundaries. As noted in the introduction F. and M. Riesz [RR] showed that every simply connected domain $\Omega$ with (1-)rectifiable boundary that harmonic measure and arclength measure are mutually absolutely continuous. Counterexamples of $\mathrm{Wu}[\mathrm{W}]$ and Ziemer [Z] show that the analogue of this theorem fails in higher dimensions.

In light of this (the counterexamples), we must impose more hypotheses in addition to rectifiability in order to ensure that surface measure is absolutely continuous with respect to harmonic measure or harmonic measure is absolutely continuous with respect to surface measure. We will show that in a particular setting we have that surface measure is absolutely continuous with respect to harmonic measure. First we show that if $K$ is the cone set for a domain then the restriction of surface measure to the cone set is absolutely continuous with respect to harmonic measure restricted to the cone set. In the plane this is part of McMillan's Theorem (see [GM, pp 207-210]) in which he shows that in a simply connected domain that the restriction of surface measure to the cone set is absolutely continuous with respect to harmonic measure restricted to the cone set. We then use this theorem to show prove that surface 
measure is absolutely continuous with respect to harmonic measure in a particular setting. This is done by showing the cone set has full surface measure. We then provide some counterexamples to show that our assumptions in this setting are not superfluous.

\subsection{Notation and Definitions}

Throughout this chapter we work in $\mathbb{R}^{n+1}, n \geq 1$. $H^{n}$ will denote the $n$-dimensional Hausdorff measure. We will work with closed sets $E \subset \mathbb{R}^{n+1}$ in which case we write $\sigma:=H^{n} \mathrm{~L} E$. We will also consider open sets $\Omega \subset \mathbb{R}^{n+1}$, not necessarily connected unless otherwise specified. In this case we shall write $\sigma:=H^{n} \mathrm{~L} \partial \Omega$.

Definition 3.1.1 (Cone Set). Given an open set $\Omega \subset \mathbb{R}^{n+1}$ we define the cone set $K=K(\Omega)$ as the set of all points $x \in \partial \Omega$ such that there exists a truncated open cone with vertex at $x$ that is contained in $\Omega$.

Definition 3.1.2 (Rectifiability). A set $E \subset \mathbb{R}^{n+1}, n \geq 1$, is called $n$-rectifiable if there exist $n$-dimensional Lipschitz maps $f_{i}: \mathbb{R}^{n} \rightarrow \mathbb{R}^{n+1}$ such that

$$
H^{n}\left(E \backslash \bigcup_{i} f_{i}\left(\mathbb{R}^{n}\right)\right)=0 .
$$

We next introduce a notion that is weaker than the well-known lower ADR condition:

Definition 3.1.4 (Weak Lower ADR (WLADR)). Let $E \subset \mathbb{R}^{n+1}, n \geq 1$, be a closed set with locally finite $H^{n}$-measure. We say the surface measure $\sigma:=$ $H^{n} L E$ satisfies the Weak Lower Ahlfors-David regular condition (WLADR) 
if $\sigma\left(E \backslash E_{*}\right)=0$ where $E_{*}$ is the relatively open set

$$
E_{*}=\left\{x \in E: \inf _{\substack{y \in B(x, \rho) \cap E \\ 0<r<\rho}} \frac{H^{n}(B(y, r) \cap E)}{r^{n}}>0 \text {, for some } \rho>0\right\} .
$$

Let us recall that $E$ is lower ADR if there exists a constant $c>0$ such that $\sigma(B(x, r) \cap E) \geq c r^{n}$ for all $x \in E$ and $r \in(0, \operatorname{diam}(E))$. Note that this is clearly stronger than WLADR. Also, if E satisfies the lower ADR condition "locally for small scales" (that is, if for every $R$ the lower ADR condition holds on $E \cap B(0, R)$, albeit with constants depending on $R$, for all $0<r<r_{R}$ for some $r_{R}<R$ ) then WLADR holds. The WLADR condition says that for $\sigma$-a.e. $x \in E$ the lower ADR condition should hold at every point in a small ball centered at $x$ and with small radius, with all constants possibly depending on $x$. This in particular allows us to deal with cusps where the lower ADR condition fails as the radius approaches 0 (see next remark). Let us finally observe that WLADR is strictly stronger than the set having positive lower density $H^{n}$-a.e.

Remark 3.1.6. There are are examples of "nice" domains whose surface measure satisfies the WLADR condition but the lower ADR and/or the lower ADR condition "locally for small scales" fail. Let $\Omega \in \mathbb{R}^{n+1}, n \geq 2$, be the domain above the graph of the function $|\cdot|^{\alpha}$ with $\alpha \in(0, \infty) \backslash\{1\}$, that is,$\Omega_{\alpha}=\left\{\left(x^{\prime}, x_{n+1}\right) \in \mathbb{R}^{n} \times \mathbb{R}: x_{n+1}>\left|x^{\prime}\right|^{\alpha}\right\}$. When $\alpha>1$ the lower ADR condition fails at 0 since $\sigma\left(B(0, r) \cap \partial \Omega_{\alpha}\right) / r^{n} \rightarrow 0$ as $r \rightarrow \infty$. However, it is easy to see that the lower ADR condition "locally for small scales" and hence WLADR follows. For $\alpha<1$, there is a cusp at 0 , and one can see that the lower ADR condition at small scales fails since $\sigma\left(B(0, r) \cap \partial \Omega_{\alpha}\right) / r^{n} \rightarrow 0$ as $r \rightarrow 0^{+}$. However, one can easily obtain the $\left(\partial \Omega_{\alpha}\right)_{*}=\partial \Omega \backslash\{0\}$ (recall the notation in Definition 3.1.4) and hence the WLADR condition holds. 
Our next definition introduces a subset of the boundary of a set in the spirit of the measure theoretic boundary (see [EG, Section 5.8]) but, here we only look at the the infinitesimal behavior from the interior.

Definition 3.1.7 (Interior Measure Theoretic Boundary). Given a set $\Omega \subset$ $\mathbb{R}^{n+1}$, the Interior Measure Theoretic Boundary $\partial_{+} \Omega$ is defined as

$$
\partial_{+} \Omega:=\left\{x \in \partial \Omega: \limsup _{r \rightarrow 0^{+}} \frac{|B(x, r) \cap \Omega|}{|B(x, r)|}>0\right\}
$$

Let us note that if an open set $\Omega$ satisfies a local (interior) corkscrew condition at $x \in \partial \Omega$, that is, if there is $0<r_{x}<\operatorname{diam}(\partial \Omega)$ and $0<c_{x}<1$ such that for every $0<r<r_{x}$ there exists $B\left(X_{B(x, r)}, c_{x} r\right) \subset B(x, r) \cap \Omega$ then clearly $x \in \partial_{+} \Omega$.

Remark 3.1.9. Consider the domains $\Omega_{\alpha}$ as in Remark 3.1.6. If $\alpha>1, \Omega_{\alpha}$ does not have interior corkscrews (for very large scales, the domain is too narrow and one cannot insert a ball of comparable radius), but it does have interior corkscrews for small scales. Hence $\partial_{+} \Omega=\partial \Omega$. When $\alpha<1$, one can see that $\partial \Omega_{+}=\partial \Omega \backslash\{0\}$ : with the exception of 0 there are interior corkscrews for small scales, but at 0 not only corkscrews fail to exist but also the limsup becomes 0 .

Definition 3.1.10 (Truncated Cones). If $z=\left(z^{\prime}, z_{n+1}\right) \in \mathbb{R}^{n+1}$ then we write $\Gamma_{h, \alpha}(z)$ for the open truncated cone with vertex at $z$, with axis $e_{n+1}$, in the direction $e_{n+1}$, with height $h>0$ and with aperture $\alpha \in(0, \pi)$, that is,

$\Gamma_{h, \alpha}(z):=\left\{y=\left(y^{\prime}, y_{n+1}\right):\left|y^{\prime}-z^{\prime}\right|<\left(y_{n+1}-z_{n+1}\right) \tan (\alpha / 2), y_{n+1} \in\left(z_{n+1}, z_{n+1}+h\right)\right\}$.

We will often suppress $\alpha$ as what will matter is that the aperture is some fixed positive number. We will sometimes use the notation $\Gamma^{+}$(in place of $\Gamma$ ) and $\Gamma^{-}$for 
the truncated cones in the direction $e_{n+1}$ and $-e_{n+1}$ respectively, this will only be necessary for the proof of Lemma 3.2.20.

The following result can be found in [M, Theorem 15.11] with the additional assumption that $H^{n}(E)<\infty$, however since the $n$-linear approximability is a local property it immediately extends to any $E$ having locally finite $H^{n}$-measure.

Theorem 3.1.11 ( $n$-linear approximability, $\left[\mathrm{M}\right.$, Theorem 15.11]). Let $E \subset \mathbb{R}^{n+1}$ be a n-rectifiable set such that $H^{n}\left\llcorner E\right.$ is locally finite. Then there exists $E_{0} \subset E$ with $H^{n}\left(E_{0}\right)=0$ such that if $x \in E \backslash E_{0}$ the following holds: for every $\eta>0$ there exist positive numbers $r_{x}=r_{x}(\eta)$ and $\lambda_{x}=\lambda_{x}(\eta)$ and a $n$-dimensional affine subspace $P_{x}=P_{x}(\eta)$ with $x \in P_{x}$ such that for all $0<r<r_{x}$

$$
H^{n}(E \cap B(y, \eta r)) \geq \lambda_{x} r^{n}, \quad \text { for } y \in P_{x} \cap B(x, r)
$$

and

$$
H^{n}\left((E \cap B(x, r)) \backslash P_{x}^{(\eta r)}\right)<\eta r^{n} .
$$

Here $P_{x}^{(\eta r)}$ is an $\eta r$-neighborhood of $P_{x}$, that is, $P_{x}^{(\eta r)}=\left\{y \in \mathbb{R}^{n+1}: \operatorname{dist}\left(y, P_{x}\right) \leq \eta r\right\}$.

Theorem 3.1.14 (Dahlberg's Theorem, [Da]). Suppose $\Omega$ is a bounded Lipschitz domain with surface measure $\sigma:=H^{n} \mathrm{~L} \partial \Omega$ then the harmonic measure associated to $\Omega, \omega$, is in $A_{\infty}(d \sigma)$. In particular, harmonic measure and surface measure are mutually absolutely continuous.

\subsection{Cone sets, harmonic measure and rectifiability}

We first prove Theorem 3.2.1 which is proved in a similar way to McMillan's Theorem. After this, the strategy of every proof will be to reduce matters to Theorem 3.2.1. 
Theorem 3.2.1. Let $\Omega \subset \mathbb{R}^{n+1}$ be an open set and let $K$ be the cone set for $\Omega$. Then there exists $\left\{\Omega_{j}\right\}$ a countable collection of bounded Lipschitz domains with $\Omega_{j} \subset \Omega$ such that $K \subset \cup_{j} \partial \Omega_{j}$.

From Theorem 3.2.1 we obtain two direct corollaries, both of which could be essentially attributed to Dahlberg (and McMillan).

Corollary 3.2.2. Let $\Omega \subset \mathbb{R}^{n+1}$ be an open set with boundary $\partial \Omega$, surface measure $\sigma:=H^{n} \mathrm{~L} \partial \Omega$ and harmonic measure $\omega$. If $K$ is the cone set and if $F \subset \partial \Omega$ is such that $\sigma(F \cap K)>0$ then there exists a point $X \in \Omega$ such that $\omega^{X}(F)>0$.

Corollary 3.2.3. Let $\Omega \subset \mathbb{R}^{n+1}$ be a connected open set (domain) with boundary $\partial \Omega$, surface measure $\sigma:=H^{n}\llcorner\partial \Omega$ and harmonic measure $\omega$. If $K$ is the cone set for $\Omega$ then $\sigma \mathrm{L} K \ll \omega\llcorner K$.

Proof of Theorem 3.2.1. Let $K$ be the cone set for $\Omega$ and $\left\{\nu_{m}\right\}$ be a countable dense subset of $\mathbb{S}^{n}$. By definition for every $x \in K$ there exists a truncated open cone with vertex $x$, axis $\nu_{x}$, height $h_{x}$ and aperture $\alpha_{x}$ which is interior to $\Omega$. For $j, m \in \mathbb{N}$ set

$$
G(j, m)=\left\{x \in K: \alpha_{x}, h_{x}>2^{-j}, \operatorname{angle}\left(\nu_{x}, \nu_{m}\right)<2^{-j-2}\right\}
$$

so that

$$
K=\bigcup_{j, m \in \mathbb{N}} G(j, m) .
$$

Then Theorem 3.2.1 will follow if we can show that each $G(j, m)$ can be covered by a countable union of boundaries of bounded Lipschitz domains which are interior to $\Omega$.

Fix $j, m \in \mathbb{N}$ and set $G=G(j, m)$. By rotation we may assume that $\nu_{m}=e_{n+1}$. It is clear that for every $x \in G$ there exists a truncated cone with vertex $x$, axis $e_{n+1}$, 
aperture $\alpha=\alpha(j)$ and height $h=h(j)$ which is interior to the original cone afforded by the fact that $x \in K$ and hence interior to $\Omega$.

Now we define "slices" $S_{\ell}$ for $\ell \in \mathbb{Z}$ as follows

$$
S_{\ell}:=\left\{X \in \mathbb{R}^{n+1}: X_{n+1} \in\left[\ell \frac{h}{10},(\ell+1) \frac{h}{10}\right)\right\}
$$

Set $F_{\ell}:=G \cap S_{\ell}$. Let $\pi$ be the projection of $\mathbb{R}^{n+1}$ onto $\mathbb{R}^{n}$ defined by $\pi(x)=$ $\pi\left(x^{\prime}, x_{n+1}\right)=x^{\prime}$. Now let $p_{j} \in \mathbb{N}$ be chosen so that the diameter of a $n$-dimensional cube of sidelength $2^{-p_{j}}$ is less than $\frac{h}{8} \tan (\alpha / 2)$ and let $\mathbb{D}_{p_{j}}$ be the collection of closed $n$-dimensional dyadic cubes with sidelength $2^{-p_{j}}$.

Claim 3.2.7. For every $Q \in \mathbb{D}_{p_{j}}$ such that $\pi^{-1}(Q) \cap F_{\ell} \neq \varnothing$,

$$
\Omega_{Q, \ell}:=\bigcup_{x \in \pi^{-1}(Q) \cap F_{\ell}} \Gamma_{h}(x) \cap\left\{\left(z^{\prime}, z_{n+1}\right) \in \mathbb{R}^{n+1}: z_{n+1}<(\ell+1) \frac{h}{10}+\frac{h}{2}\right\}
$$

is a bounded star-shaped domain with respect to a ball and hence a bounded Lipschitz domain.

Proof of Claim 4.3.20. Without loss of generality we may assume $\ell=-1$. Let $y_{Q}$ be the center of $Q$ and set $Y_{Q}=\left(y_{Q}, \frac{h}{4}\right)$. Take an arbitrary $x=\left(x^{\prime}, x_{n+1}\right) \in \pi^{-1}(Q) \cap F_{\ell}$. Since

$$
\Gamma_{\max }(x):=\Gamma_{h}(x) \cap\left\{\left(z^{\prime}, z_{n+1}\right) \in \mathbb{R}^{n+1}: z_{n+1}<\frac{h}{2}\right\}
$$

is convex, it suffices to show that $B\left(Y_{Q}, R\right) \subset \Gamma_{\max }(x)$ for some $R$ independent of $x$. Note that $\Gamma_{\frac{h}{2}}\left(x^{\prime}, 0\right) \subset \Gamma_{\max }(x)$, so we instead show that $B\left(Y_{Q}, R\right) \subset \Gamma_{\frac{h}{2}}\left(x^{\prime}, 0\right)$ for some $R$ independent of $x^{\prime}$. Recall that

$$
\Gamma_{\frac{h}{2}}\left(x^{\prime}, 0\right)=\left\{\left(z^{\prime}, z_{n+1}\right):\left|z^{\prime}-x^{\prime}\right|<z_{n+1} \tan (\alpha / 2), z_{n+1} \in\left(0, \frac{h}{2}\right)\right\}
$$


so that $K_{x^{\prime}}=\left\{\left(z^{\prime}, \frac{h}{4}\right):\left|z^{\prime}-x^{\prime}\right| \leq \frac{h}{8} \tan (\alpha / 2)\right\}$ is a compact subset of $\Gamma_{\frac{h}{2}}\left(x^{\prime}, 0\right)$. Set

$$
R:=\frac{1}{2} \operatorname{dist}\left(K_{x^{\prime}}, \partial \Gamma_{\frac{h}{2}}\left(x^{\prime}, 0\right)\right)>0
$$

and notice that $R$ has no dependence on $x^{\prime}$. Also, by choice of $p_{j}$, we have that $\left|y_{Q}-x^{\prime}\right| \leq \frac{h}{8} \tan (\alpha / 2)$. Hence $Y_{Q} \in K_{x^{\prime}}$ and $B\left(Y_{Q}, R\right) \subset \Gamma_{\frac{h}{2}}\left(x^{\prime}, 0\right) \subset \Gamma_{\max }(x)$ as desired. For a proof that bounded star-shaped domains with respect to a ball are bounded Lipschitz domains see [Maz, Section 1.1.8].

Once the claim is proved we observe that by construction, $\pi^{-1}(Q) \cap F_{\ell} \subset \partial \Omega_{Q, \ell}$, to see this we need only to observe that if $z_{1}, z_{2} \in F_{\ell}$ then $z_{2} \notin \Gamma_{h}\left(z_{1}\right)$, since $\Gamma_{h}\left(z_{1}\right)$ does not meet $E$. Then we have that

$$
G=\bigcup_{\ell} F_{\ell} \subset \bigcup_{\ell} \bigcup_{Q \in \mathbb{D}_{p_{j}}} \partial \Omega_{Q, \ell}
$$

where we take $\Omega_{Q, \ell}=\varnothing$ if $\pi^{-1}(Q) \cap F_{\ell}=\varnothing$. This completes the proof.

Now we prove Corollaries 3.2.2 and 3.2.3, each of which are a direct consequence of Theorem 3.2.1.

Proof of Corollary 3.2.2. Let $\Omega \subset \mathbb{R}^{n+1}$ be a open set with boundary $\partial \Omega$, surface measure $\sigma:=H^{n} \mathrm{~L} \partial \Omega$ and harmonic measure $\omega$. Let $K$ be the cone set for $\Omega$ and suppose that $F \subset \mathbb{R}^{n+1}$ is a Borel set such that $\sigma(F \cap K)>0$. By Theorem 3.2.1 there exists $\left\{\Omega_{j}\right\}$ a countable collection of bounded Lipschitz domains with $\Omega_{j} \subset \Omega$ such that $K \subset \cup_{j} \partial \Omega_{j}$. It follows that since $\sigma(F \cap K)>0$ that there exists a bounded Lipschitz domain $\Omega_{j}$ such that $\sigma\left(\partial \Omega_{j} \cap F \cap K\right)>0$. Fix $X \in \Omega_{j}$ and note that also $X \in \Omega$. Dahlberg's Theorem (Theorem 3.1.14) gives that $H^{n} \mathrm{~L} \partial \Omega_{j}$ is absolutely continuous with respect to $\omega_{\partial \Omega_{j}}^{X}$, the harmonic measure for $\Omega_{j}$ with pole at $X$. It 
follows that $\omega_{\Omega_{j}}^{X}\left(\partial \Omega_{j} \cap F \cap K\right)>0$, the maximum principle then yields that

$$
\omega^{X}(F) \geq \omega^{X}(F \cap K) \geq \omega_{\Omega_{j}}^{X}\left(\partial \Omega_{j} \cap F \cap K\right)>0,
$$

which proves the Corollary.

Proof of Corollary 3.2.3. Let $\Omega \subset \mathbb{R}^{n+1}$ be a open set with boundary $\partial \Omega$, surface measure $\sigma:=H^{n} \mathrm{~L} \partial \Omega$ and harmonic measure $\omega$. Let $K$ be the cone set for $\Omega$ and suppose that $F \subset \mathbb{R}^{n+1}$ is a Borel set such that $\sigma(F \cap K)>0$. It is clear that $\Omega$ satisfies the hypothesis of Corollary 3.2.2 so that there exists an $X \in \Omega$ such that $\omega^{X}(F \cap K)>0$. Since $\Omega$ is connected it follows by the Harnack inequality that $\omega^{X}(F \cap K)>0$ for any $X$ in $\Omega$. Thus

$$
\sigma \mathrm{L} K(F)>0 \Longrightarrow \omega\llcorner K(F)>0
$$

so that $\sigma \mathrm{L} K \ll \omega\llcorner K$.

Before we continue, we prove two auxiliary Lemmas which allow us to establish settings in which the cone set for a domain has full surface measure.

Lemma 3.2.15 (Existence of Truncated Cones). Let $E \subset \mathbb{R}^{n+1}$ be a $n$-rectifiable set with locally finite surface measure, write $\sigma:=H^{n} \mathrm{~L} E$ and use the notation in Theorem 3.1.11. Given $x \in E \backslash E_{0}$ assume that there exists $\rho_{x}, c_{x}>0$ such that

$$
\sigma(B(y, r) \cap E) \geq c_{x} r^{n}, \quad \forall y \in B(x, \rho) \cap E, 0<r \leq \rho_{x}
$$

For every $0<\eta<\eta_{0}\left(c_{x}\right):=\min \left\{2^{-4 n}, c_{x}^{2}\right\}$, there exists a two sided truncated cone with vertex at $x$, height $h(\eta):=\eta^{\frac{1}{4 n}} \min \left\{r_{x}(\eta), \rho_{x}\right\}$ and aperture $\alpha(\eta):=2 \arctan \left(\eta^{-\frac{1}{4 n}} / 2\right)>$ $\pi / 2$ which does not meet $E$. (Note that $\alpha(\eta) \rightarrow \pi$ as $\eta \rightarrow 0^{+}$.) 
Proof. Without loss of generality we may take $x=0$ and $P_{x}=\mathbb{R}^{n} \times\{0\}$. Given $0<\eta<\eta_{0}\left(c_{x}\right)$ we are going to see that $\Gamma_{h(\eta), \alpha(\eta)}(0) \subset \mathbb{R}^{n+1} \backslash E$ with $h(\eta)$ and $\alpha(\eta)$ are in the statement. Notice that $\alpha(\eta)>\pi / 2$ from the choice of $\eta$ and also that $\alpha(\eta) \rightarrow \pi$ as $\eta \rightarrow 0^{+}$. A similar argument shows existence of similar truncated cone in the direction of $-e_{n+1}$. Suppose (for the the sake of contradiction) that there exist $r \in(0, h(\eta))$ and $z \in D_{r} \cap E$ where

$$
D_{r}=\left\{z=\left(z^{\prime}, z_{n+1}\right): z_{n+1}=\eta^{\frac{1}{4 n}} r,\left|z^{\prime}\right|<\frac{r}{2}\right\}
$$

Then since $\eta_{0} \leq 2^{-4 n}$ it follows that

$$
E \cap B\left(z, \eta^{\frac{1}{2 n}} r\right) \subset(E \cap B(0, r)) \backslash P_{x}^{(\eta r)} .
$$

On the other hand, the fact that $\eta_{0} \leq c_{x}^{2}$ yields

$$
\sigma\left(E \cap B\left(z, \eta^{\frac{1}{2 n}} r\right)\right) \geq c_{x} \eta^{\frac{1}{2}} r^{n}>\eta r^{n},
$$

which together with (3.2.18) contradicts (3.1.13).

Lemma 3.2.20 (Existence of Interior Truncated Cones). Let $\Omega$ be an open set and whose $n$-rectifiable boundary, $\partial \Omega$, has locally finite surface measure $H^{n} \mathrm{~L} \partial \Omega$. Assume that $x \in \partial \Omega \backslash(\partial \Omega)_{*}$ (recall the notation in Definition 3.1.4) satisfies the hypothesis of Lemma 3.2.15 with $E=\partial \Omega, \rho_{x}$ and $c_{x}$ as above. Given $\epsilon>0$ there exists $\tilde{\eta}_{0}=\tilde{\eta}_{0}(\epsilon)<\eta_{0}\left(c_{x}\right)$ such that if $0<\eta<\tilde{\eta}_{0}$ and

$$
\limsup _{r \rightarrow 0^{+}} \frac{|B(x, r) \cap \Omega|}{|B(x, r)|}>\epsilon
$$

then one of the cones constructed in Lemma 3.2.15 must be in the interior of $\Omega$. 
Proof. We may assume again that $x=0$ and $P_{x}=\mathbb{R}^{n} \times\{0\}$ and let $0<\eta<\tilde{\eta}_{0}<$ $\eta_{0}\left(c_{x}\right)$, where $\tilde{\eta}_{0}$ is to be chosen momentarily. If $0<r<h(\eta)$ then by a rescaling argument

$$
\frac{\left|B(x, r) \backslash\left(\Gamma_{h(\eta), \alpha(\eta)}^{+} \cup \Gamma_{h(\eta), \alpha(\eta)}^{-}\right)\right|}{|B(x, r)|}=\frac{\left|B(x, h(\eta)) \backslash\left(\Gamma_{h(\eta), \alpha(\eta)}^{+} \cup \Gamma_{h(\eta), \alpha(\eta)}^{-}\right)\right|}{|B(x, h(\eta))|}
$$

and since $\alpha(\eta) \rightarrow \pi$ as $\eta \rightarrow 0^{+}$one sees that

$$
\frac{\left|B(x, h(\eta)) \backslash\left(\Gamma_{h(\eta), \alpha(\eta)}^{+} \cup \Gamma_{h(\eta), \alpha(\eta)}^{-}\right)\right|}{|B(x, h(\eta))|} \downarrow 0
$$

as $\eta \rightarrow 0^{+}$. Choosing $\tilde{\eta}_{0}$ sufficiently small (depending on $\epsilon$ ) we have that

$$
\frac{\left|B(x, h(\eta)) \backslash\left(\Gamma_{h(\eta), \alpha(\eta)}^{+} \cup \Gamma_{h(\eta), \alpha(\eta)}^{-}\right)\right|}{|B(x, h(\eta))|}<\epsilon / 2
$$

for any fixed $0<\eta<\tilde{\eta}_{0}$. On the other hand by (3.2.21) there exists $0<r<h(\eta)$ such that

$$
\frac{|B(x, r) \cap \Omega|}{|B(x, r)|}>\epsilon .
$$

It follows from (3.2.22), (3.2.24) and (3.2.25) that at least one of the cones must meet $\Omega$. Recall that neither of the cones meet $E=\partial \Omega$, hence one of the cones must be interior.

We are now ready to prove Theorems 3.2.28, 3.2.26 and 3.2.31, all of which we achieve by showing that the cone set for the given domain or open set has full $H^{n}$ measure.

Theorem 3.2.26. Let $E \subset \mathbb{R}^{n+1}, n \geq 1$, be a closed set with locally finite $H^{n}$-measure satisfying the WLADR condition. Then, $E$ is n-rectifiable if and only if there exists a countable collection $\left\{\Omega_{j}\right\}$ of bounded Lipschitz domains with $\Omega_{j} \subset \mathbb{R}^{n+1} \backslash E$ for every 
$j$, and a set $Z \subset E$ with $H^{n}(Z)=0$ such that

$$
E \subset Z \cup\left(\bigcup_{j} \partial \Omega_{j}\right)
$$

The innovation in Theorem 3.2.26 is the fact that each $\Omega_{j}$ is contained in $\mathbb{R}^{n+1} \backslash E$, otherwise this would be the standard covering of a rectifiable set by Lipschitz graphs.

Proof of Theorem 3.2.26. If (3.2.27) holds then it clearly $E$ is $n$-rectifiable. Let $\Omega:=$ $\mathbb{R}^{n+1} \backslash E$, so that $\partial \Omega=E$. By Theorem 3.2.1 it is enough to show that there exists a set $Z \subset \partial \Omega$ such that $H^{n}(Z)=0$ and $K \subset \partial \Omega \backslash Z$ where $K$ is the cone set for $\Omega$. We will use the notation of Theorem 3.1.11, and Lemma 3.2.15.

Let $E_{0}$ be as in Theorem 3.1.11 and $E_{*}$ be as in Definition 3.1.4. Then by hypothesis (and Theorem 3.1.11) $H^{n}\left(E_{0}\right)=0$ and $H^{n}\left(\partial \Omega \backslash E_{*}\right)=0$, so that $H^{n}\left(E_{0} \cup(\partial \Omega \backslash\right.$ $\left.\left.E_{*}\right)\right)=0$. We set $Z=E_{0} \cup\left(\partial \Omega \backslash E_{*}\right)$ and show for each $x \in \partial \Omega \backslash Z$ there exists a truncated cone with vertex $x$, interior to $\partial \Omega$. If $x \in \partial \Omega \backslash Z$ then $x \in E \backslash E_{0}$ and $x \in E_{*}$ so that there exist $c_{x}$ and $\rho_{x}$ such that (3.2.16) holds. Thus by Lemma 3.2.15 there exists a truncated cone which does not meet $E$ and hence is interior to $\Omega$. This proves the theorem.

By the same argument we prove the next theorem using Corollary 3.2.2.

Theorem 3.2.28. Let $E \subset \mathbb{R}^{n+1}, n \geq 1$, be a closed set with locally finite $H^{n}$-measure satisfying the "weak lower ADR" (WLADR) condition. Under these background hypotheses, if $E$ is n-rectifiable then $\left.H^{n}\right|_{E}$ is "absolutely continuous" with respect to harmonic measure for $\mathbb{R}^{n+1} \backslash E$, in the sense that if $F \subset E$ is a Borel set with $H^{n}(F)>0$, then $\omega^{X}(F)>0$ for some $X \in \mathbb{R}^{n+1} \backslash E$. 
Remark 3.2.29. Let us note that in the previous result the "absolute continuity" property needs to be interpreted properly, as we are comparing one measure $\sigma$ with the collection of harmonic measures $\left\{\omega^{X}\right\}_{X \in \mathbb{R}^{n+1} \backslash E}$. An equivalent formulation of the conclusion is that if $F \subset E$ is a Borel set with $\omega^{X}(F)=0$ for every $X \in \mathbb{R}^{n+1} \backslash E$, then necessarily $H^{n}(F)=0$. One can restate this in terms of genuine absolute continuity of $H^{n} \mathrm{~L} E$ with respect to an averaged harmonic measure:

$$
H^{n}\left\llcorner E \ll \widetilde{\omega}:=\sum_{k \geq 1} 2^{-k} \omega_{k}\right.
$$

where $\omega_{k}=\omega_{D_{k}}^{X_{k}}$ is the harmonic measure for the domain $D_{k}$ with some fixed pole $X_{k} \in D_{k}$, and $\left\{D_{k}\right\}_{k \geq 1}$ is an enumeration of the connected components of $\mathbb{R}^{n+1} \backslash E$.

Proof of Theorem 3.2.28. Let $\Omega=\mathbb{R}^{n+1} \backslash E$, so that $\partial \Omega=E$ and set $\sigma:=H^{n} L \partial \Omega$. By the proof of Theorem 3.2.26 we have that $\sigma(\partial \Omega \backslash K)=0$. It then follows that $\sigma \mathrm{L} K=\sigma$. Then by Corollary 3.2.2

$$
\sigma=\sigma L K \ll \omega L K \ll \omega
$$

which proves the theorem.

Now we prove Theorem 3.2.31 which is essentially a refinement of Theorem 3.2.28 and Theorem 3.2.26.

Theorem 3.2.31. Let $\Omega \subset \mathbb{R}^{n+1}, n \geq 1$, be an open connected set, whose boundary $\partial \Omega$ has locally finite $H^{n}$-measure. Assume that $\partial \Omega$ satisfies the WLADR condition. Assume further that $H^{n}\left(\partial \Omega \backslash \partial_{+} \Omega\right)=0$ where $\partial_{+} \Omega$ is the Interior Measure Theoretic Boundary. Then, $\partial \Omega$ is n-rectifiable if and only if there exists a countable collection $\left\{\Omega_{j}^{\mathrm{int}}\right\}_{j}$ of bounded Lipschitz domains with $\Omega_{j}^{\mathrm{int}} \subset \Omega$ for every $j$, and a set $Z \subset \partial \Omega$ 
with $H^{n}(Z)=0$ such that

$$
\partial \Omega \subset Z \cup\left(\bigcup_{j} \partial \Omega_{j}^{\mathrm{int}}\right)
$$

As a consequence, if $\partial \Omega$ is $n$-rectifiable (and $\Omega$ satisfies the background hypothesis above) $H^{n}\left\llcorner\partial \Omega\right.$ is absolutely continuous with respect to $\omega$, where $\omega=\omega^{X}$ is the harmonic measure for $\Omega$ with some (or any) fixed pole $X \in \Omega$.

Remark 3.2.33. The connectivity assumption is, in a way, cosmetic. If $\Omega$ were an open set rather than a domain the conclusion would be that $\left.H^{n}\right|_{\partial \Omega}$ is "absolutely continuous" with respect to $\omega$ in the sense that if $F \subset \partial \Omega$ is a Borel set with $H^{n}(F)>$ 0 then $\omega^{X}(F)>0$ for some $X \in \Omega$ (or any $X$ in the same connected component).

Remark 3.2.34. Note that (3.2.32) implies that $\partial \Omega \backslash \partial_{+} \Omega \subset Z$ and hence condition $\sigma\left(\partial \Omega \backslash \partial_{+} \Omega\right)=0$ is necessary for the approximation of $\Omega$ by interior Lipschitz subdomains.

Proof of Theorem 3.2.31. As in the proof of Theorem 3.2.26 to show (3.2.32) it is enough to show that there exists a set $Z \subset \partial \Omega$ such that $H^{n}(Z)=0$ and $K \subset \partial \Omega \backslash Z$.

To this effect, let $\partial \Omega_{0}, \partial \Omega_{*}$ and $\partial \Omega_{+}$be as in Theorem 3.1.11, definition 3.1.4 and definition 3.1 .7 respectively (where $E=\partial \Omega$ in the definition of $\partial \Omega_{0}, \partial \Omega_{*}$ ). By hypothesis $H^{n}\left(\partial \Omega_{0}\right)=H^{n}\left(\partial \Omega \backslash \partial \Omega_{*}\right)=H^{n}\left(\partial \Omega \backslash \partial \Omega_{+}\right)=0$. We set

$$
Z=\partial \Omega_{0} \cup\left(\partial \Omega \backslash \partial \Omega_{*}\right) \cup\left(\partial \Omega \backslash \partial \Omega_{+}\right),
$$

so that $H^{n}(Z)=0$. We are left with showing that if $x \in \partial \Omega \backslash Z$ then there exists a truncated cone at $\mathrm{x}$ which is interior to $\partial \Omega$. Let $x \in \partial \Omega \backslash Z$, then $x \in \partial \Omega_{+}$so that there exists an $\epsilon>0$ such that

$$
\limsup _{r \rightarrow 0^{+}} \frac{|B(x, r) \cap \Omega|}{|B(x, r)|}>\epsilon>0 .
$$


Additionally, since $x \in \partial \Omega \backslash \partial \Omega_{0}$ and $x \in \partial \Omega_{*}$ so that there exist positive numbers $c_{x}$ and $\rho_{x}$ satisfying (3.2.16). Choosing $\eta(\epsilon)$ as in Lemma 3.2.20 we then have that there exists a truncated cone with vertex $x$ interior to $\Omega$. This proves (3.2.32). Let $\sigma=H^{n}\left\llcorner\partial \Omega\right.$. To show that $\sigma \ll \omega$, again we have that $H^{n}(\partial \Omega \backslash K) \leq H^{n}(Z)=0$, so that by Corollary 3.2.3 it follows that

$$
\sigma=\sigma\llcorner K \ll \omega L K \ll \omega .
$$

This shows that $\sigma \ll \omega$ and proves the Theorem.

We conclude this section with Theorem 3.2.38 which is a direct Corollary of Theorem 3.2.31 combined with $\left[\mathrm{AHM}^{3} \mathrm{TV}\right.$, Theorem 1.1].

Theorem 3.2.38. Let $\Omega \subset \mathbb{R}^{n+1}, n \geq 1$, be an open connected set, whose boundary $\partial \Omega$ has locally finite $H^{n}$-measure. Assume that $\partial \Omega$ satisfies the WLADR condition and that the Interior Measure Theoretic Boundary has full $H^{n}$-measure. Then $\Omega$ is $n$-rectifiable if and only if $\left.H^{n}\right|_{\partial \Omega}$ is absolutely continuous with respect to $\omega$, where $\omega=\omega^{X}$ is the harmonic measure for $\Omega$ with some (or any) fixed pole $X \in \Omega$.

Proof of Theorem 3.2.38. Let $\Omega \subset \mathbb{R}^{n+1}, n \geq 1$, be an open connected set, whose boundary $\partial \Omega$ has locally finite $H^{n}$-measure. Assume that $\partial \Omega$ satisfies the WLADR condition and that the Interior Measure Theoretic Boundary has full $H^{n}$-measure. Set $\sigma=H^{n}\llcorner\partial \Omega$.

It follows immediately from Theorem 3.2.31 that if $\partial \Omega$ is $n$-rectifiable $\sigma \ll \omega$. The other direction follows from $\left[\mathrm{AHM}^{3} \mathrm{TV}\right.$, Theorem 1.1(b)] which states that if $\Omega \subset \mathbb{R}^{n+1}$ is an open connected set and $E \subset \partial \Omega$ is a set of finite $H^{n}$ measure such that $H^{n} L E \ll \omega$ then $E$ is rectifiable. In our setting we apply the theorem with 
$E=\partial \Omega$ and note that the Theorem extends to sets which have the property that $H^{n} \mathrm{~L} E$ is locally finite.

\subsection{Counterexamples}

In this section we produce examples of domains with rectifiable boundaries for which surface measure fails to be absolutely continuous with respect to harmonic measure. The first example is a domain that does not have locally finite perimeter and the second one, based on a construction presented by Jonas Azzam in January 2015 at ICMAT (Spain), fails to satisfy the WLADR condition. These examples show that in Theorem 3.2.31 we cannot drop any of our background hypotheses. Let us point out that the assumption $\partial \Omega$ being $n$-rectifiable is also necessary by Theorem 3.2.38 (see also $[\mathrm{ABaHM}])$. As we will observe below the same constructions allow us to obtain that in Theorem 3.2.31 we cannot also drop any of our background hypotheses.

In what follows, for a given domain $\Omega$ we will use the notation $\omega_{\Omega}^{X}$ for the harmonic measure for $\Omega$ with pole at $X \in \Omega$. In both examples we make use of the maximum principle, that is, if $X \in \Omega^{\prime} \subset \Omega$ and $F \subset \partial \Omega$ then

$$
\omega_{\Omega^{\prime}}^{X}\left(F \cap \partial \Omega^{\prime}\right) \leq \omega_{\Omega}^{X}(F)
$$

Example 3.3.2. For $k \geq 1$, and $n \geq 1$, set

$$
\Sigma_{k}=\left\{(x, t) \in \mathbb{R}_{+}^{n+1}: t=2^{-k},|x| \geq 2^{-k}\right\}
$$

and define

$$
\Omega:=\mathbb{R}_{+}^{n+1} \backslash\left(\cup_{k=1}^{\infty} \Sigma_{k}\right), \quad \Omega_{k}:=\mathbb{R}_{+}^{n+1} \backslash \Sigma_{k}, \quad \Omega_{k}^{\prime}:=\mathbb{R}^{n} \times\left(2^{-k}, \infty\right) .
$$

Then $\Omega$ is an open connected domain whose boundary clearly does not have locally 
finite $H^{n}$-measure (any surface ball centered at $\mathbb{R}^{n} \times\{0\}$ contains infinitely many $n$ dimensional balls of fixed radius). It is immediate to see that $\partial \Omega$ satisfies the WLADR condition as $(\partial \Omega)_{*}=\partial \Omega$ (recall the notation in Definition 3.1.4). Notice also that $\Omega$ satisfies the interior Corkscrew condition (as the sets $\Sigma_{k}$ are located at heights which are separate enough) and hence $\partial \Omega_{+}=\partial \Omega$. Finally $\partial \Omega=\left(\mathbb{R}^{n} \times\{0\}\right) \cup\left(\cup_{k=1}^{\infty} \Sigma_{k}\right)$ which is $n$-rectifiable.

Take $X^{*}=(0, \ldots, 0,2) \in \Omega$ and we are going show that $\omega_{\Omega}^{X^{*}}(F)=0$ with $F=$ $\mathbb{R}^{n} \times\{0\} \subset \partial \Omega$. Since $\Omega \subset \Omega_{k},(3.3 .1)$ implies that $\omega_{\Omega}^{X^{*}}(F) \leq \omega_{\Omega_{k}}^{X^{*}}(F)$, hence we just need to see that $\omega_{\Omega_{k}}^{X^{*}}(F) \rightarrow 0$ as $k \rightarrow \infty$. Write $\Delta_{k}^{\prime}=\left\{(x, t) \in \mathbb{R}_{+}^{n+1}: t=2^{-k},|x|<\right.$ $\left.2^{-k}\right\} \subset \partial \Omega_{k}^{\prime}$. Using the fact that harmonic measures for $\Omega_{k}$ and $\Omega_{k}^{\prime}$ are probabilities, that $\Omega_{k}^{\prime} \subset \Omega_{k}$ and maximum principle (3.3.1) we see that

$$
\omega_{\Omega_{k}}^{X^{*}}(F)=1-\omega_{\Omega_{k}}^{X^{*}}\left(\partial \Omega_{k} \backslash F\right)=1-\omega_{\Omega_{k}}^{X^{*}}\left(\Sigma_{k}\right) \leq 1-\omega_{\Omega_{k}^{\prime}}^{X^{*}}\left(\Sigma_{k}\right)=\omega_{\Omega_{j}^{\prime}}^{X^{*}}\left(\Delta_{k}^{\prime}\right) .
$$

Since $\Omega_{k}^{\prime}$ is a translation of $\mathbb{R}_{+}^{n+1}$ we can use the classical Poisson kernel for the upper-half space $P(x, t)$ and one has that

$$
\omega_{\Omega_{k}^{\prime}}^{X^{*}}\left(\Delta_{k}^{\prime}\right)=\int_{|y|<2^{-k}} P\left(y, 2-2^{-k}\right) d y \rightarrow 0, \quad \text { as } k \rightarrow \infty .
$$

This shows that surface measure fails to be absolutely continuous with respect to harmonic measure $\omega_{\Omega}^{X^{*}}$ and hence with respect to $\omega_{\Omega}^{X}$ for every $X \in \Omega$ since $\Omega$ is connected.

To summarize, we have constructed $\Omega$, an open connected set, satisfying all the conditions in Theorem 3.2.31 with the exception that $\partial \Omega$ has locally finite $H^{n}$ measure, and for which the conclusion of Theorem 3.2.31 fails.

Remark 3.3.4. If we repeat the same construction of Example 3.3.2 in the lower halfspace and let $E$ be the boundary of the resulting open set (which has now 2 connected 
components), then clearly $E$ satisfies all the hypotheses in Theorem 3.2.28, except for $E$ having locally finite $H^{n}$-measure. In this case we can analogously prove that for the same set $F$ as before $\omega^{X}(F)=0$ for every $X \in \mathbb{R}^{n+1} \backslash E$, hence the conclusion of Theorem 3.2.28 does not hold.

Example 3.3.5. For $k \geq 1$, and $n \geq 2$, set

$$
\Sigma_{k}:=\left\{(x, t) \in \mathbb{R}_{+}^{n+1}: t=2^{-k}, x \in \overline{\Delta\left(0,2^{-k} c_{k}\right)}+c_{k} \mathbb{Z}^{n}\right\},
$$

where $c_{k} \downarrow 0$ will be chosen, and for $x \in \mathbb{R}^{n}, \Delta(x, r):=\left\{y \in \mathbb{R}^{n}:|x-y|<r\right\}$ is the usual $n$-disk of radius $r$ centered at $x$. Define

$$
\Omega:=\mathbb{R}_{+}^{n+1} \backslash\left(\cup_{k=1}^{\infty} \Sigma_{k}\right), \quad \Omega_{k}:=\mathbb{R}_{+}^{n+1} \backslash \Sigma_{k},
$$

which is clearly open and connected. Notice that $\Omega$ satisfies the interior Corkscrew condition (note that the sets $\Sigma_{k}$ are located at heights which are separate enough), hence $\partial \Omega_{+}=\partial \Omega$.

We assume that $c_{k}$ decays rapidly enough. It is easy to see that $\partial \Omega$ satisfies the upper ADR condition. Also, the WLADR (and hence the lower ADR) fails. To see this, given $X=(x, 0) \in \partial \Omega$, we can find a sequence of points $X_{k}=\left(c_{k} \vec{l}_{k, x}, 2^{-k}\right) \rightarrow X$ with $\vec{l}_{k, x} \in \mathbb{Z}^{n}$. But then, for $k$ large enough $H^{n}\left(B\left(X_{k}, 2^{-k-2}\right) \cap \partial \Omega\right) / r^{n} \approx c_{k} \rightarrow 0$ as $k \rightarrow \infty$. Hence $\mathbb{R}^{n} \times\{0\} \subset(\partial \Omega)_{*}$ (recall the notation in Definition 3.1.4) and the WLADR condition fails.

Let $\omega^{(\cdot)}:=\omega_{\Omega}^{(\cdot)}$ and $\omega_{k}^{(\cdot)}:=\omega_{\Omega_{k}}^{(\cdot)}$ denote harmonic measure for the domains $\Omega$ and $\Omega_{k}$ respectively.

Claim. If $c_{k}$ decays fast enough, then $\omega^{(\cdot)}(F)=0$, with $F=\mathbb{R}^{n} \times\{0\}$.

Assuming this momentarily we have defined $\Omega$, an open connected set, satisfying 
all the conditions in Theorem 3.2.31 with the exception of the WLADR property, and for which the conclusion of Theorem 3.2.31 fails. Again, as in Remark 3.3.4, we may obtain a counterexample for Theorem 3.2.28 that satisfies all its hypotheses but the WLADR condition.

Before proving our claim we need to recall some definitions. Given $O \subset \mathbb{R}^{n+1}$ an open and $K$ a compact subset of $O$ we define the capacity of $K$ relative to $O$ as

$$
\operatorname{cap}(K, O)=\inf \left\{\iint_{O}|\nabla \phi|^{2} d Y: \phi \in C_{0}^{\infty}(O), \phi \geq 1 \text { in } K\right\} .
$$

Also, the inhomogeneous capacity of $K$ is defined as

$$
\operatorname{Cap}(K)=\inf \left\{\iint_{\mathbb{R}^{n+1}}\left(|\phi|^{2}+|\nabla \phi|^{2}\right) d Y: \phi \in C_{0}^{\infty}(\mathbb{R}), \phi \geq 1 \text { in } K\right\}
$$

Combining [HKM, Theorem 2.38], [AH, Theorem 2.2.7] and [AH, Theorem 4.5.2] we have that if $K$ is a compact subset of $\bar{B}$, where $B$ is a ball with radius smaller than 1 , then

$$
\operatorname{cap}(K, 2 B) \gtrsim \operatorname{Cap}(K) \gtrsim \sup _{\mu} \frac{\mu(K)^{2}}{\|W(\mu)\|_{L^{1}(\mu)}}
$$

where the implicit constants depend only on $n$, the sup runs over all Radon positive measures supported on $K$; and

$$
W(\mu)(X):=\int_{0}^{1} \frac{\mu(B(X, t))}{t^{n-1}} \frac{d t}{t}, \quad X \in \operatorname{supp} \mu .
$$

We are now ready to prove our claim. We fix $k \geq 2$, take $c_{k}=2^{-2 k n}$ and write $N=N_{k}:=c_{k}^{-1}>1$. We are going to show that

$$
\operatorname{cap}\left(\overline{B\left(X_{0}, s\right)} \cap \Sigma_{k}, B\left(X_{0}, 2 s\right)\right) \gtrsim s^{n-1}, \quad X_{0}:=\left(x_{0}, 2^{-k}\right) \in \Sigma_{k}, N^{-1 / 2} \leq s<1 .
$$


For a fixed $X_{0}$ and $s$, write $K=\overline{B\left(X_{0}, s\right)} \cap \Sigma_{k}$ and set $\mu=\left.2^{k n} s^{-1} H^{n}\right|_{K}$, and note that for $X \in K$

$$
\mu(B(X, r)) \approx 2^{k n} s^{-1} \begin{cases}r^{n}, & r<2^{-k} N^{-1}, \\ 2^{-k n} N^{-n}, & 2^{-k} N^{-1} \leq r \leq N^{-1} \\ 2^{-k n} r^{n}, & N^{-1}<r \leq s \\ 2^{-k n} s^{n}, & r>s .\end{cases}
$$

To compute $W(\mu)(X)$ for $X \in K$ write

$$
W(\mu)(X)=\int_{0}^{2^{-k} N^{-1}}+\int_{2^{-k} N^{-1}}^{N^{-1}}+\int_{N^{-1}}^{s}+\int_{s}^{1}=: I+I I+I I I+I V .
$$

Then, since $s \geq N^{-1 / 2}$,

$$
I+I I \lesssim 2^{k n} s^{-1}\left(2^{-k} N^{-1}+2^{-k n} N^{-n} \int_{2^{-k} N^{-1}}^{\infty} \frac{d r}{r^{n}}\right) \lesssim 2^{k(n-1)} N^{-1 / 2} \lesssim 1
$$

where the last bound holds by our choice of $N$ and $c_{k}$. Furthermore, the last two estimates in (3.3.8) easily imply that $I I I+I V \lesssim 1$ and hence $W(\mu)(X) \lesssim 1$ for every $X \in K$. This, (3.3.6), and (3.3.8) imply as desired (3.3.7):

$$
\operatorname{cap}\left(\overline{B\left(X_{0}, s\right)} \cap \Sigma_{k}, B\left(X_{0}, 2 s\right)\right) \gtrsim \mu(K) \gtrsim s^{n-1}
$$

Set

$$
P_{k}:=\left\{\left(x, 2^{-k}-N^{-1 / 2}\right) \in \mathbb{R}_{+}^{n+1}: x \in \mathbb{R}^{n}\right\},
$$

and observe that for $X \in P_{k}$,

$$
N^{-1 / 2} \leq \delta_{k}(X):=\operatorname{dist}\left(X, \partial \Omega_{k}\right)=\operatorname{dist}\left(X, \Sigma_{k}\right) \leq 2 N^{-1 / 2}
$$

We now define

$$
u(X):=\omega_{k}^{X}(F), \quad X \in \Omega_{k},
$$

and observe that $u \in W^{1,2}\left(\Omega_{k}\right) \cap C\left(\overline{\Omega_{k}}\right)$ since $\partial \Omega_{k}$ is ADR (constants depend on $k$ but we just use this qualitatively) and $1_{F}$ is a Lipschitz function on $\partial \Omega_{k}$. Fix 
$Z_{0} \in P_{k}$ and let $Z_{0}^{\prime} \in \Sigma_{k}$ be such that $\left|Z_{0}-Z_{0}^{\prime}\right|=\operatorname{dist}\left(Z_{0}, \partial \Omega_{k}\right) \leq 2 N^{-1 / 2}$. Let $\Omega_{Z_{0}}=\Omega_{k} \cap B\left(Z_{0}^{\prime}, \frac{3}{4} 2^{-k}\right)$, which is an open connected bounded set. We can now apply [HKM, Example 2.12, Theorem 6.18] to obtain $\alpha=\alpha(n)>0$ such that

$$
u\left(Z_{0}\right) \lesssim \exp \left(-\alpha \int_{3 N^{-1 / 2}}^{2^{-k-2}} \frac{d s}{s}\right) \approx\left(2^{k} N^{-1 / 2}\right)^{\alpha}=2^{-\alpha k(n-1)} .
$$

where we have used $u \equiv 0$ on $\partial \Omega_{k} \cap B\left(Z_{0}^{\prime}, 2^{-k-1}\right)$ and (3.3.7). Note that the last estimate holds for any $Z_{0} \in P_{k}$ and therefore, by the maximum principle,

$$
u(x, t) \lesssim 2^{-\alpha k(n-1)}, \quad(x, t) \in \Omega_{k}, t>2^{-k}-N^{-1 / 2} .
$$

In particular, if we set $X_{0}:=(0, \ldots, 0,1) \in \mathbb{R}_{+}^{n+1}$, then by another application of the maximum principle,

$$
\omega^{X_{0}}(F) \leq \omega_{k}^{X_{0}}(F)=u\left(X_{0}\right) \lesssim 2^{-\alpha k(n-1)} \rightarrow 0,
$$

as $k \rightarrow \infty$, and the claim is established. 


\section{Chapter 4}

\section{A Two Phase Free Boundary Problem}

In Chapters 2 and 3 we looked at how boundary regularity can yield desirable properties for harmonic measure. In this chapter we look in the other direction we hope to gain boundary regularity provided that the Poisson kernel has sufficient regularity. In this chapter we look at a two-phase free boundary problem. We show that (under some background hypothesis) weak regularity of the Poisson kernel on either side of a domain yields the same weak regularity for, $\nu$, the outer unit normal to the domain.

\subsection{Notation and Definitions}

Throughout this chapter $\Omega \subset \mathbb{R}^{n+1}, n \geq 2$, will be a domain. We will also assume that $\Omega_{e x t}:=\mathbb{R}^{n+1} \backslash \bar{\Omega}$ is domain. We now recall the relevant definitions for this section.

Definition 4.1.1. (Poisson Kernel) Given a domain $\Omega \subset \mathbb{R}^{n+1}$ and a point $X \in \Omega$, let $\omega^{X}$ be the harmonic measure for $\Omega$ with pole at $X$ and $\sigma:=H^{n}\llcorner\partial \Omega$ be the surface measure for $\Omega$. If $\omega^{X} \ll \sigma$ then we call the Radon-Nikodym derivative of $\omega^{X}$ with respect to $\sigma$ the Poisson kernel with pole at $X$.

Definition 4.1.2. (Riesz transforms and the single layer potential) Let $E \subset$ 
$\mathbb{R}^{n+1}$ be an $n$-dimensional ADR (hence closed) set with surface measure $\sigma$. We define the (vector valued) Riesz kernel as

$$
\mathcal{K}(x)=\tilde{c}_{n} \frac{x}{|x|^{n+1}}
$$

where $\tilde{c}_{n}$ is chosen so that $\mathcal{K}$ is the gradient of fundamental solution to the Laplacian. For a Borel measurable function $f$, we then define the Riesz transform

$$
\mathcal{R} f(X):=\mathcal{K} *(f \sigma)(X)=\int_{E} \mathcal{K}(X-y) f(y) d \sigma(y) \quad X \in \mathbb{R}^{n+1}
$$

as well as the truncated Riesz transforms

$$
\mathcal{R}_{\varepsilon} f(X):=\int_{E \cap\{|X-y|>\varepsilon\}} \mathcal{K}(X-y) f(y) d \sigma(y), \quad \varepsilon>0 .
$$

We define $\mathcal{S}$ the single layer potential for the Laplacian relative to $E$ to be

$$
\mathcal{S} f(X):=\int_{E} \mathcal{E}(X-y) f(y) d \sigma(y)
$$

where $\mathcal{E}(X)=c_{n}|X|^{1-n}$ is the (positive) fundamental solution to the Laplacian in $\mathbb{R}^{n+1}$. Notice that $\nabla \mathcal{S} f(X)=\mathcal{R} f(X)$ for $X \notin E$.

Remark 4.1.6. Recall Definition 2.1.3. We note that the principal value $\mathcal{R} f(x)=$ $\lim _{\varepsilon \rightarrow 0} \mathcal{R}_{\varepsilon} f(x)$ exists for a.e. $x \in E$, provided that $E$ is rectifiable, thus, in particular, if $E$ is UR (see, e.g., [M, Theorem 20.28]). Of course, the $L^{2}$ bound in (2.1.4) holds also for the principal value operator. Moreover, by standard Calderón-Zygmund theory, the $L^{2}$ bound self-improves to give $L^{p}$ bounds, $1<p<\infty$.

Definition 4.1.7. (UR domain). Following the terminology in [HMT], we will say that a domain $\Omega$ is a UR domain if $\partial \Omega$ is UR, and if the measure theoretic boundary $\partial_{*} \Omega$ (see Definition 4.1 .10 below) satisfies $\sigma\left(\partial \Omega \backslash \partial_{*} \Omega\right)=0$. 
Definition 4.1.8. (2-sided NTA). Recalling definition 2.1.11, we say that a domain $\Omega \subset \mathbb{R}^{n+1}$ is NTA ("Non-tangentially accessible") if it satisfies the Harnack Chain condition, and if both $\Omega$ and $\Omega_{\mathrm{ext}}:=\mathbb{R}^{n+1} \backslash \bar{\Omega}$ satisfy the interior Corkscrew condition (see Definition 2.1.8). If $\Omega$ and $\Omega_{\text {ext }}$ both NTA domains then we say $\Omega$ is a 2 -sided NTA domain.

Definition 4.1.9. (Chord arc domain and 2-sided Chord arc domain). We say that a domain $\Omega \subset \mathbb{R}^{n+1}$ is a chord arc (resp. 2-sided chord arc) domain, if $\Omega$ is an NTA (resp. 2-sided NTA) domain, and $\partial \Omega$ is n-dimensional ADR. Note that in either case $\Omega$ is a UR domain.

Definition 4.1.10. (Measure theoretic boundary). Given $\Omega \subset \mathbb{R}^{n+1}$, a set of locally finite perimeter ${ }^{1}$, we say that $x \in \partial_{*} \Omega$, the measure theoretic boundary of $\Omega$, if

$$
\limsup _{r \rightarrow 0} \frac{|B(x, r) \cap \Omega|}{r^{n+1}}>0
$$

and

$$
\limsup _{r \rightarrow 0} \frac{\left|B(x, r) \cap \Omega^{c}\right|}{r^{n+1}}>0
$$

where $|A|$ is the Lebesgue measure of $A$. Given a domain $\Omega \subseteq \mathbb{R}^{n+1}$ we say that the measure theoretic boundary has full measure if $H^{n}\left(\partial \Omega \backslash \partial_{*} \Omega\right)=0$. One should note that if $\Omega \subset \mathbb{R}^{n+1}$ is a set of locally finite perimeter then the measure theoretic boundary and the reduced boundary differ by a set of $H^{n}$ measure zero, so it then follows that the measure theoretic boundary has full measure if and only the reduced boundary has full measure (see [EG, Section 5.8]).

\footnotetext{
${ }^{1}$ We note that if $\partial \Omega$ is ADR (in particular, if it is UR), then $\Omega$ has locally finite perimeter, by the criterion in [EG, Theorem 1, p. 222].
} 


\section{Definition 4.1.13. (Nontangential approach region and maximal function).}

Fix $\alpha>0$ and let $\Omega$ be a domain then for $x \in \partial \Omega$ we define the nontangential approach region (or "cone")

$$
\Gamma(x)=\Gamma_{\alpha}(x)=\{Y \in \Omega:|Y-x|<(1+\alpha) \delta(Y)\} .
$$

We also define the nontangential maximal function for $u: \Omega \rightarrow \mathbb{R}$

$$
\mathcal{N} u(x)=\mathcal{N}_{\alpha} u(x)=\sup _{Y \in \Gamma_{\alpha}(x)}|u(Y)|, \quad x \in \partial \Omega .
$$

We make the convention that $\mathcal{N} u(x)=0$ when $\Gamma_{\alpha}(x)=\varnothing$.

Definition 4.1.16. (VMO and $\mathrm{VMO}_{l o c}$ ). Let $E \subset \mathbb{R}^{n+1}$ be $n$-dimensional ADR, and let $\sigma:=\left.H^{n}\right|_{E}$ as above. We denote by $V M O(d \sigma)=V M O(E, d \sigma)$ the closure of the set of bounded uniformly continuous functions defined on $E$ in $B M O(E, d \sigma)$. We say that $f \in V M O_{l o c}(d \sigma)$ if

$$
\lim _{r \rightarrow 0} \sup _{x \in K} f_{\Delta(x, r)}\left|f(x)-f_{\Delta(x, r)} f\right| d \sigma=0,
$$

for every compact $K \subset E$. Of course, it is well known that (4.1.17) holds with $E$ in place of $K$ in the supremum, if and only if $f \in V M O(E, d \sigma)$. Thus, $V M O$ and $V M O_{l o c}$ are distinct only for unbounded $E$.

We now record some estimates and some known results that will be important in the proofs of the theorems.

\subsection{Preliminary Estimates and Observations}

In the sequel, we will sometimes assume more on $\Omega$ and $\Omega_{\mathrm{ext}}$, however $\Omega$ and $\Omega_{\mathrm{ext}}$ will always be UR domains. In addition, $k_{1}$ will be the Poisson kernel for the domain $\Omega$ and $k_{2}$ will be the Poisson kernel for $\Omega_{\text {ext }}$. 
Suppose that $\log k_{1}^{X_{1}} \in V M O_{l o c}(d \sigma)$. Let $\omega=\omega^{X_{1}}$ and $k=k^{X_{1}}$ be the harmonic measure and Poisson Kernel for $\Omega$ with pole at $X_{1}$ (resp.). Then by the definition of $V M O_{l o c}(d \sigma)$, for each $B_{0}:=B\left(x_{0}, r_{0}\right)$, with $x_{0} \in \partial \Omega$, and $r_{0}>0$, and for each $\eta>0$, there exists $r_{1}>0$ such that if $x \in \partial \Omega \cap B_{0}$, and $s \in\left(0, r_{1}\right)$, then

$$
f_{\Delta(x, s)}\left|\log k(z)-f_{\Delta(x, s)} \log k(y) d \sigma(y)\right| d \sigma(z) \leq \eta .
$$

Since $\partial \Omega$ is ADR, it follows that $k$ and $k^{-1}$ belong to $R H_{q, l o c}(d \sigma)$ ("local Reverse Hölder- $q$ "), for every $q<\infty$; i.e., given $B_{0}=B\left(x_{0}, r_{0}\right)$, for every $q \in(1, \infty)$, there exists $C_{q, B_{0}}$ such that for any surface ball $\Delta=\Delta(x, r)$, with $x \in B_{0} \cap \partial \Omega$, and $r \leq r_{0}$,

$$
\left(f_{\Delta} k^{q} d \sigma\right)^{1 / q} \leq C_{q, B_{0}} f_{\Delta} k d \sigma
$$

and

$$
\left(f_{\Delta} k^{-q} d \sigma\right)^{1 / q} \leq C_{q, B_{0}} f_{\Delta} k^{-1} d \sigma .
$$

See, e.g., [KT3, Theorem 2.1, p. 332], or the references cited there. As a consequence of (4.2.2) and (4.2.3) (holding for all $q<\infty$ ) we have the following Lemma.

Lemma 4.2.4 ([KT3] Corollary 2.4). Suppose that $\log k \in V M O_{l o c}(d \sigma)$. Fix $B_{0}:=B\left(x_{0}, r_{0}\right)$, with $x_{0} \in \partial \Omega$ and $r_{0}>0$. Then for all $\beta>0, w \in B_{0} \cap \partial \Omega, s \leq r_{0}$, and $E \subset \Delta(w, s)=: \Delta$,

$$
C_{\beta, B_{0}}^{-1}\left(\frac{\sigma(E)}{\sigma(\Delta)}\right)^{1+\beta} \leq \frac{\omega(E)}{\omega(\Delta)} \leq C_{\beta, B_{0}}\left(\frac{\sigma(E)}{\sigma(\Delta)}\right)^{1-\beta} .
$$

The reverse Hölder estimate (4.2.2) for some fixed $q>1$ (i.e., the $A_{\infty}$ property) yields an exponential reverse Jensen inequality, so that for any $\Delta$ as in (4.2.2),

$$
e^{f_{\Delta} \log k d \sigma} \approx f_{\Delta} k d \sigma=\frac{\omega(\Delta)}{\sigma(\Delta)},
$$


with implicit constants that may depend on $B_{0}$. See [GR, Theorem 2.15, p. 405] for a proof of (4.2.6). For the connection between $A_{\infty}$ and BMO, see [GR, Corollary 2.19, p. 409].

We shall require also the following.

Lemma 4.2.7. Let $B_{0}:=B\left(x_{0}, r_{0}\right)$, with $x_{0} \in \partial \Omega$ and $r_{0}>0$. Given $\epsilon \in(0,1)$, let $r_{1}>0$ be such that (4.2.1) holds for all $x \in \partial \Omega \cap B_{0}$ and $s \in\left(0, r_{1}\right)$, with $\eta=\epsilon$. Let $p \in(1, \infty)$ and suppose that $M r \in\left(0, r_{1}\right)$. If we set $\Delta^{*}:=\Delta(x, M r)$, and $a:=a_{x, M r}=e^{f_{\Delta^{*}} \log k d \sigma}$, one then has

$$
\left(f_{\Delta^{*}}\left|1-\frac{k}{a}\right|^{p} d \sigma\right)^{1 / p} \leq C_{1} \epsilon^{b},
$$

with $b=b(p)=(4 p)^{-1}$, and for some $C_{1}=C_{1}\left(p, B_{0}\right)$, uniformly for $x \in \partial \Omega \cap B_{0}$ and $r<r_{1} / M$.

Remark. Applying (4.2.6) with $\Delta^{*}$ in place of $\Delta$, we note for future reference that

$$
a \approx \frac{\omega(\Delta(x, M r))}{\sigma(\Delta(x, M r))},
$$

Proof. Set $A:=\left\{z \in \Delta^{*}:\left|\log k(z)-f_{\Delta^{*}} \log k(y) d \sigma(y)\right|>\sqrt{\epsilon}\right\}$, so that by Chebyshev's inequality one has

$$
\sigma(A) \leq \sqrt{\epsilon} \sigma\left(\Delta^{*}\right) .
$$

Set $F:=\Delta^{*} \backslash A$, then by definition of $a$, we have for $z \in F$,

$$
|\log k(z)-\log a| \leq \sqrt{\epsilon} .
$$

Exponentiating (4.2.11) we obtain

$$
e^{-\sqrt{\epsilon}} \leq \frac{k(z)}{a} \leq e^{\sqrt{\epsilon}},
$$


so that by Taylor's Theorem one has

$$
\left|1-\frac{k(z)}{a}\right| \leq C \sqrt{\epsilon}, \quad \forall z \in F
$$

Then we have

$$
\begin{aligned}
&\left(f_{\Delta^{*}} \mid 1-\right.\left.\left.\frac{k}{a}\right|^{p} d \sigma\right)^{1 / p} \\
& \leq\left(\frac{1}{\sigma\left(\Delta^{*}\right)} \int_{F}\left|1-\frac{k}{a}\right|^{p} d \sigma\right)^{1 / p}+\left(\frac{1}{\sigma\left(\Delta^{*}\right)} \int_{A}\left|1-\frac{k}{a}\right|^{p} d \sigma\right)^{1 / p} \\
& \\
& \lesssim \sqrt{\epsilon}+\left(\frac{1}{\sigma\left(\Delta^{*}\right)} \int_{A}\left|1-\frac{k}{a}\right|^{p} d \sigma\right)^{1 / p}
\end{aligned}
$$

Using Minkowski's inequality, (4.2.10), (4.2.9), and that $k \in R H_{2 p}$ one has

$$
\begin{aligned}
\left(\frac{1}{\sigma\left(\Delta^{*}\right)} \int_{A}\left|1-\frac{k}{a}\right|^{p} d \sigma\right)^{1 / p} & \leq\left(\frac{\sigma(A)}{\sigma\left(\Delta^{*}\right)}\right)^{1 / p}+\frac{1}{a}\left(\frac{1}{\sigma\left(\Delta^{*}\right)} \int_{A} k^{p}\right)^{1 / p} \\
& \leq \epsilon^{\frac{1}{2 p}}+\left(\frac{\sigma(A)}{\sigma\left(\Delta^{*}\right)}\right)^{\frac{1}{2 p}} \frac{1}{a}\left(\frac{1}{\sigma\left(\Delta^{*}\right)} \int_{A} k^{2 p} d \sigma\right)^{1 / 2 p} \\
& \leq \epsilon^{\frac{1}{2 p}}+\epsilon^{\frac{1}{4 p}} \frac{1}{a} f_{\Delta^{*}} k d \sigma \lesssim \epsilon^{\frac{1}{4 p}}
\end{aligned}
$$

where the implicit constants may depend on $B_{0}$. Then, since $p>1$, putting (4.2.14) and (4.2.15) together we obtain (4.2.8), with $C_{1}$ depending on $B_{0}$ and $p$.

Since $\partial \Omega$ is $\mathrm{UR}$, for all $p \in(1, \infty)$ we have

$$
\|\mathcal{N}(\nabla \mathcal{S} f)\|_{p} \leq C\|f\|_{p}
$$

where $C$ depends on the UR character of $\partial \Omega$, dimension, $p$, and the aperture of the cones defining $\mathcal{N}$. Estimate (4.2.16) is essentially proved in [DS1] (bounds for the nontangential maximal function of $\nabla \mathcal{S} f$ follow from uniform bounds for the truncated singular integrals, plus a standard Cotlar Lemma argument; the details may be found in [HMT, Proposition 3.20].)

In addition, we have the following result proved in [HMT]. 
Lemma 4.2.17 ([HMT, Proposition 3.30]). , If $\Omega$ is a UR domain (recall Definition 4.1.7), then for a.e. $x \in \partial \Omega$, and for all $f \in L^{p}(d \sigma), 1<p<\infty$,

$$
\lim _{\substack{Z \rightarrow x \\ Z \in \Gamma^{-}(x)}} \nabla \mathcal{S} f(Z)=-\frac{1}{2} \nu(x) f(x)+T f(x),
$$

and

$$
\lim _{\substack{Z \rightarrow x \\ Z \in \Gamma^{+}(x)}} \nabla \mathcal{S} f(Z)=\frac{1}{2} \nu(x) f(x)+T f(x) .
$$

where $T$ is a principal value singular integral operator, $\Gamma^{+}(x)$ is the cone at $x$ relative to $\Omega, \Gamma^{-}(x)$ is the cone at $x$ relative to $\Omega_{\mathrm{ext}}$, and $\nu$ is the outer normal to $\Omega$.

Remark 4.2.20. We have taken our fundamental solution to be positive, so for that reason there are some changes in sign in both (4.2.18) and (4.2.19) as compared to the formulation in $[\mathrm{HMT}]$.

Remark 4.2.21. Recall that by Definition 4.1.7, we have assumed in particular that $\sigma\left(\partial \Omega \backslash \partial \Omega_{*}\right)=0$, and therefore the measure theoretic outer unit normal $\nu$ exists a.e. on $\partial \Omega$ (see [EG, Chapter 5]).

We recall now some fundamental estimates relating harmonic measure and the Green function.

Lemma 4.2.22 ([JK])). Let $\Omega \subset \mathbb{R}^{n+1}$ be an NTA domain and let $G(X, Y)$ denote the Green function of $\Omega$. Let $x \in \partial \Omega, R \in(0$, diam $\partial \Omega)$, and let $Y_{R}$ be a corkscrew point for $B(x, R)$. If $X \in \Omega \backslash B(x, 2 R)$, then

$$
G\left(X, Y_{R}\right) \approx \frac{\omega^{X}(\Delta(x, R))}{R^{n-1}} .
$$

Moreover, if $g(Y)$ is the Green function with pole at infinity, and $\omega$ is harmonic measure associated to g (see [KT4]) then

$$
g\left(Y_{R}\right) \approx \frac{\omega(\Delta(x, R))}{R^{n-1}} .
$$


The implicit constants in (4.2.23)-(4.2.24) depend only on dimension and the NTA constants.

Remark. In the case that the pole is finite (estimate (4.2.23)), this result was proved in $[\mathrm{JK}]$; the result for pole at infinity follows from the finite case, plus the construction in [KT2]. Estimate (4.2.23) has been extended to general divergence form elliptic operators in [CFMS].

Lemma 4.2.25 ([JK]). Let $\Omega$ be an NTA domain and suppose that $u$ is harmonic in $\Omega$ and vanishes continuously on $\Delta(x, 2 r)$ then

$$
\sup _{X \in B(x, r) \cap \Omega} u(X) \lesssim u\left(Y_{x, r}\right)
$$

where $Y_{x, r}$ is a corkscrew point for $\Delta(x, r)$. The implicit constants depend only on the NTA constants and dimension.

We also recall a result proved by Bourgain about harmonic measure on domains with ADR boundary.

Lemma 4.2.27 ([Bo]). Let $\Omega \subset \mathbb{R}^{n+1}$ be an open set with $n$-dimensional $A D R$ boundary $\partial \Omega$. Then there exists constants $C_{0}>2$, and $c_{1}>0$, depending on dimension and $A D R$ such that for all $x_{1} \in \partial \Omega$ and $R \in(0, \operatorname{diam}(\partial \Omega))$, if $Y \in \Omega \cap B\left(x_{1}, R\right)$ then

$$
\omega^{Y}\left(B\left(x_{1}, C_{0} R\right)\right) \geq c_{1}
$$

\subsection{Theorems 4.3.1 and 4.3.18}

We are now ready to state and prove the main theorems of this Chapter.

Theorem 4.3.1. Let $\Omega$ and $\Omega_{\mathrm{ext}}:=\mathbb{R}^{n+1} \backslash \bar{\Omega}$ be connected domains in $\mathbb{R}^{n+1}$, whose common boundary $\partial \Omega=\partial \Omega_{\text {ext }}$ is uniformly rectifiable, and whose measure theoretic 
boundary $\partial_{*} \Omega$ satisfies $\sigma\left(\partial \Omega \backslash \partial_{*} \Omega\right)=0$. Suppose that there is some fixed pair of points $X_{1} \in \Omega$ and $X_{2} \in \Omega_{\mathrm{ext}}$, such that $\log k_{1}^{X_{1}}, \log k_{2}^{X_{2}} \in V M O_{\text {loc }}(d \sigma)$, where $k_{1}$ and $k_{2}$ are the Poisson kernels for $\Omega$ and $\Omega_{\text {ext }}$ respectively. Then $\nu \in V M O_{l o c}(d \sigma)$.

Thus, uniform rectifiability of the boundary, along with the hypothesis that the measure theoretic boundary has full measure, replace the stronger 2-sided chord arc condition $^{2}$, at least in the case that our Poisson kernels have finite poles. On the other hand, in the case that the Poisson kernel has pole at infinity, we will impose an NTA hypothesis, as in [KT4] (see Theorem 4.3.18).

Proof of Theorem 4.3.1. Fix $B_{0}=B\left(x_{0}, r_{0}\right)$, and $B_{0}^{\star}:=B\left(x_{0}, r_{0}^{\star}\right)$, with $x_{0} \in \partial \Omega$, $r_{0}>0$, and $r_{0}^{\star}=100 C_{0}\left(\left|X_{1}-x_{0}\right|+r_{0}+1\right)$, where $C_{0}$ is the constant in Lemma 4.2.27. Fix also $\epsilon \in(0,1)$, and let $r_{1}>0$ be such that (4.2.1) holds with $\eta=\epsilon$, for $x \in \partial \Omega \cap B_{0}^{\star}$ and $s \in\left(0, r_{1}\right)$. Without loss of generality we take $r_{1}<\min \left\{\frac{\delta\left(X_{1}\right)}{50}, 1, r_{0}\right\}$. Let $M:=50 \epsilon^{-\frac{1}{8 n}}$, and suppose that $M r \in\left(0, r_{1}\right)$. We now fix $x \in B_{0} \cap \partial \Omega$, and for any points $y, z \in \Delta(x, r)$, let $y^{*}, z^{*}$ denote arbitrary points in $\Gamma^{-}(y) \cap B(y, r / 2)$ and in $\Gamma^{-}(z) \cap B(z, r / 2)$, respectively. Setting

$$
\Delta:=\Delta(x, r), \quad \Delta^{*}:=\Delta(x, M r),
$$

we shall first prove that for any such $y, z, y^{*}, z^{*}$,

$$
\left(f_{\Delta}\left|\nabla \mathcal{S} 1_{\Delta^{*}}\left(z^{*}\right)-f_{\Delta} \nabla \mathcal{S} 1_{\Delta^{*}}\left(y^{*}\right) d \sigma(y)\right|^{2} d \sigma(z)\right)^{\frac{1}{2}} \leq C \epsilon^{\gamma},
$$

where $C=C\left(n, U R, B_{0}, B_{0}^{\star}, \delta\left(X_{1}\right),\left|X_{1}-x_{0}\right|\right)$, and where $\gamma$ is a positive constant, depending only on dimension. For the sake of notational convenience, in the sequel,

\footnotetext{
${ }^{2}$ It is known that chord arc domains have uniformly rectifiable boundaries [DJ], and in fact, the chord arc condition is strictly stronger.
} 
we shall often allow generic and implicit constants to depend upon these parameters, without explicitly making note of such dependence. As before, set $a:=a_{x, M r}=$ $e^{f_{\Delta^{*}} \log k d \sigma}$ and write

$$
1_{\Delta^{*}}=\left[\left(1-\frac{k}{a}\right) 1_{\Delta^{*}}\right]+\left[\frac{k}{a}\right]-\left[\left(\frac{k}{a}\right) 1_{(\Delta *)^{c}}\right] .
$$

Using (4.3.3) we have that the left hand side of (4.3.2) is bounded by the sum of three terms $I, I I$ and $I I I$ where

$$
\begin{gathered}
I=\left(f_{\Delta}\left|\nabla \mathcal{S}\left[\left(1-\frac{k}{a}\right) 1_{\Delta^{*}}\right]\left(z^{*}\right)-f_{\Delta} \nabla \mathcal{S}\left[\left(1-\frac{k}{a}\right) 1_{\Delta^{*}}\right]\left(y^{*}\right) d \sigma(y)\right|^{2} d \sigma(z)\right)^{\frac{1}{2}}, \\
I I=\left(f_{\Delta}\left|\nabla \mathcal{S}\left[\frac{k}{a}\right]\left(z^{*}\right)-f_{\Delta} \nabla \mathcal{S}\left[\frac{k}{a}\right]\left(y^{*}\right) d \sigma(y)\right|^{2} d \sigma(z)\right)^{\frac{1}{2}},
\end{gathered}
$$

and

$$
I I I=\left(f_{\Delta}\left|\nabla \mathcal{S}\left[\left(\frac{k}{a}\right) 1_{(\Delta *)^{c}}\right]\left(z^{*}\right)-f_{\Delta} \nabla \mathcal{S}\left[\left(\frac{k}{a}\right) 1_{(\Delta *)^{c}}\right]\left(y^{*}\right) d \sigma(y)\right|^{2} d \sigma(z)\right)^{\frac{1}{2}} .
$$

We begin by estimating $I$. By (4.2.16) and Lemma 4.2 .7 with $p=2$, we have

$$
\begin{aligned}
I \leq 2\left(f_{\Delta}\left|\mathcal{N}\left(\nabla \mathcal{S}\left[\left(1-\frac{k}{a}\right) 1_{\Delta^{*}}\right]\right)\right|^{2} d \sigma\right)^{\frac{1}{2}} & \\
& \lesssim M^{\frac{n}{2}}\left(f_{\Delta^{*}}\left|1-\frac{k}{a}\right|^{2} d \sigma\right)^{\frac{1}{2}} \lesssim M^{\frac{n}{2}} \epsilon^{\frac{1}{8}} \lesssim \epsilon^{\frac{1}{16}}
\end{aligned}
$$

Now for $I I$, we recall that $k=k_{1}^{X_{1}}$ is harmonic measure for $\Omega$ with pole at $X_{1}$. Moreover, $\mathcal{E}\left(\cdot-z^{*}\right)$ and $\mathcal{E}\left(\cdot-y^{*}\right)$ are harmonic in $\Omega$ since $z^{*}, y^{*} \in \Omega_{\mathrm{ext}}$, and decay to 0 at infinity, and are therefore equal to their respective Poisson integrals in $\Omega$. Consequently,

$$
I I=\frac{1}{a}\left(f_{\Delta} f_{\Delta}\left|\nabla \mathcal{E}\left(X_{1}-z^{*}\right)-\nabla \mathcal{E}\left(X_{1}-y^{*}\right) d \sigma(y)\right|^{2} d \sigma(z)\right)^{\frac{1}{2}} .
$$


Recall that by definition, $M r+r_{0}<2 r_{0} \ll r_{0}^{\star}$, and that $x \in B_{0} \cap \partial \Omega$, hence $B(x, M r) \subset 2 B_{0} \subset B_{0}^{\star}$. Thus, we may apply Lemma 4.2 .4 , with $r_{0}^{\star}$, $B_{0}^{\star}$ in place of $r_{0}, B_{0}$, with $w=x_{0}$ and $s=r_{0}^{\star}$ (thus, $B(w, s)=B_{0}^{\star}, \Delta=\Delta_{0}^{\star}=B_{0}^{\star} \cap \partial \Omega$ ), and with $E=\Delta(x, M r)$, to deduce that

$$
\frac{\omega\left(\Delta_{0}^{\star}\right)}{\omega(\Delta(x, M r))} \lesssim\left(\frac{r_{0}^{\star}}{M r}\right)^{n+\tau}, \quad \forall \tau>0
$$

where the implicit constant of course depends upon $\tau$ and $B_{0}^{\star}$.

We note that by Lemma 4.2.27, and the definition of $r_{0}^{\star}$, there is a uniform constant $c>0$ such that

$$
\omega\left(\Delta_{0}^{\star}\right) \geq c .
$$

We further note that, since $y^{*}, z^{*} \in B(x, 2 r)$,

$$
\left|\nabla \mathcal{E}\left(X_{1}-z^{*}\right)-\nabla \mathcal{E}\left(X_{1}-y^{*}\right)\right| \lesssim \frac{r}{\delta\left(X_{1}\right)^{n+1}}
$$

Then continuing (4.3.8), we have, using (4.2.9), (4.3.9) (with $\tau=1 / 2$ ), and (4.3.10),

$$
\begin{aligned}
& I I \lesssim \frac{1}{a} \frac{r}{\delta\left(X_{1}\right)^{n+1}} \approx \frac{\sigma(\Delta(x, M r))}{\omega(\Delta(x, M r))} \frac{r}{\delta\left(X_{1}\right)^{n+1}} \\
&=\frac{\sigma(\Delta(x, M r))}{\omega\left(\Delta_{0}^{\star}\right)} \frac{\omega\left(\Delta_{0}^{\star}\right)}{\omega(\Delta(x, M r))} \frac{r}{\delta\left(X_{1}\right)^{n+1}} \\
& \lesssim(M r)^{n}\left(\frac{r_{0}^{\star}}{M r}\right)^{n+\frac{1}{2}} \frac{r}{\delta\left(X_{1}\right)^{n+1}} \lesssim M^{-\frac{1}{2}} r^{\frac{1}{2}} \lesssim \frac{1}{M} \approx \epsilon^{\frac{1}{8 n}}
\end{aligned}
$$

since $r<r_{1} / M \ll \delta\left(X_{1}\right) / M$, where we remind the reader that in this part of the argument, we allow implicit constants to depend upon $\delta\left(X_{1}\right)$, and on the various parameters involved in the definition of $r_{0}^{\star}$.

For III we use basic Calderón-Zygmund type estimates as follows. Let

$$
\Delta_{j}^{\prime}:=\Delta\left(x, 2^{j} r\right), \quad A_{j}^{\prime}:=\Delta_{j}^{\prime} \backslash \Delta_{j-1}^{\prime},
$$


so that

$$
\begin{aligned}
& I I I= \\
& \left(f_{\Delta}\left|f_{\Delta}\left(\nabla \mathcal{S}\left[\left(\frac{k}{a}\right) 1_{(\Delta *)^{c}}\right]\left(z^{*}\right)-\nabla \mathcal{S}\left[\left(\frac{k}{a}\right) 1_{(\Delta *)^{c}}\right]\left(y^{*}\right)\right) d \sigma(y)\right|^{2} d \sigma(z)\right)^{\frac{1}{2}} \\
& =\left(f_{\Delta}\left|f_{\Delta} \int_{\partial \Omega \backslash \Delta^{*}}\left[\nabla \mathcal{E}\left(z^{*}-w\right)-\nabla \mathcal{E}\left(y^{*}-w\right)\right] \frac{k(w)}{a} d \sigma(w) d \sigma(y)\right|^{2} d \sigma(z)\right)^{\frac{1}{2}} \\
& \leq \sum_{j: 2^{j} \geq M}\left(f_{\Delta}\left[f_{\Delta} \int_{A_{j}^{\prime}}\left|\nabla \mathcal{E}\left(z^{*}-w\right)-\nabla \mathcal{E}\left(y^{*}-w\right)\right| \frac{k(w)}{a} d \sigma(w) d \sigma(y)\right]^{2} d \sigma(z)\right)^{\frac{1}{2}} \\
& \lesssim \sum_{j: 2^{j} \geq M}\left(f_{\Delta}\left[f_{\Delta} \int_{A_{j}^{\prime}} \frac{r}{\left(2^{j} r\right)^{n+1}} \frac{k(w)}{a} d \sigma(w) d \sigma(y)\right]^{2} d \sigma(z)\right)^{\frac{1}{2}} \\
& =\sum_{j \in J_{1}} \ldots+\sum_{j \in J_{2}} \ldots=: I I I_{1}+I I I_{2}, \quad(4.3 .12)
\end{aligned}
$$

where

$$
J_{1}:=\left\{j: M \leq 2^{j} \leq r_{0}^{\star} / r\right\}, \quad J_{2}:=\left\{j: 2^{j}>r_{0}^{\star} / r\right\} .
$$

For $j \in J_{1}$, we may apply Lemma 4.2.4, with $B_{0}^{\star}$ in place of $B_{0}$, and with $\Delta=\Delta_{j}^{\prime}$ and $E=\Delta(x, M r)$, to obtain

$$
\frac{\omega\left(\Delta_{j}^{\prime}\right)}{\omega(\Delta(x, M r))} \lesssim\left(\frac{2^{j}}{M}\right)^{n+\beta n}
$$

We then have

$$
\begin{aligned}
& I I I_{1} \lesssim \sum_{j \in J_{1}} \frac{1}{2^{j}}\left(f_{\Delta}\left[f_{\Delta} f_{\Delta_{j}^{\prime}} \frac{k(w)}{a} d \sigma(w) d \sigma(y)\right]^{2} d \sigma(z)\right)^{\frac{1}{2}} \\
& \lesssim \sum_{j \in J_{1}} \frac{1}{2^{j}} \frac{1}{a} \frac{\omega\left(\Delta_{j}^{\prime}\right)}{\sigma\left(\Delta_{j}^{\prime}\right)} \approx \sum_{j \in J_{1}} \frac{1}{2^{j}} \frac{\sigma(\Delta(x, M r))}{\sigma\left(\Delta_{j}^{\prime}\right)} \frac{\omega\left(\Delta_{j}^{\prime}\right)}{\omega(\Delta(x, M r))} \\
& \lesssim \sum_{j: 2^{j} \geq M} 2^{-j}\left(\frac{M}{2^{j}}\right)^{n}\left(\frac{2^{j}}{M}\right)^{n+1 / 2} \lesssim \frac{1}{M} \lesssim \epsilon \frac{1}{8 n}
\end{aligned}
$$

where in the middle line we have used (4.2.9), and in the last line, the ADR property and (4.3.13) with $\beta=1 /(2 n)$. Since $\omega$ is a probablility measure, we also have that 


$$
\begin{aligned}
& I I I_{2} \lesssim \sum_{j: 2^{j} r>r_{0}^{\star}} r\left(2^{j} r\right)^{-n-1} a^{-1} \approx r\left(\frac{1}{r_{0}^{\star}}\right)^{n+1} \frac{\sigma(\Delta(x, M r))}{\omega(\Delta(x, M r))} \\
& =r\left(\frac{1}{r_{0}^{\star}}\right)^{n+1} \frac{\sigma(\Delta(x, M r))}{\omega\left(\Delta_{0}^{*}\right)} \frac{\omega\left(\Delta_{0}^{*}\right)}{\omega(\Delta(x, M r))} \lesssim r\left(\frac{1}{r_{0}^{\star}}\right)^{n+1}(M r)^{n}\left(\frac{r_{0}^{\star}}{M r}\right)^{n+1 / 2} \\
& =\left(\frac{r}{r_{0}^{\star}}\right)^{1 / 2} M^{-1 / 2} \lesssim M^{-1} \approx \epsilon^{\frac{1}{8 n}}
\end{aligned}
$$

where in the middle line we have used the ADR property, (4.3.10), and (4.3.9) (with $\tau=1 / 2)$, and in the last line that $M r \leq r_{1} \ll r_{0}^{\star}$.

Combining the estimates for $I, I I, I I I_{1}$ and $I I I_{2}$, we obtain (4.3.2) with $\gamma=$ $1 /(8 n)$ (or with $\gamma=1 / 16$, if $n=1$, see $(4.3 .7))$.

Setting

$$
\text { n.t. } \nabla \mathcal{S}^{-} f(x):=\lim _{\substack{Z \rightarrow x \\ Z \in \Gamma^{-}(x)}} \nabla \mathcal{S} f(Z)
$$

since the limit exists for a.e. $x \in \partial \Omega$ (see Lemma 4.2.17), we may now use (4.2.16), (4.3.2), and dominated convergence to obtain

$$
\left(f_{\Delta} \mid n . t . \nabla \mathcal{S}^{-} 1_{\Delta^{*}}(z)-f_{\Delta} \text { n.t. }\left.\nabla \mathcal{S}^{-} 1_{\Delta^{*}}(y) d \sigma(y)\right|^{2} d \sigma(z)\right)^{\frac{1}{2}} \leq C \epsilon^{\gamma}
$$

for $x \in \partial \Omega \cap B_{0}$ and $r<r_{1} / M$. In addition since $\log k_{2}^{X_{2}} \in V M O(d \sigma)$ the same analysis shows that $(4.3 .14)$ holds for $n . t . \nabla \mathcal{S}^{-} 1_{\Delta^{*}}$ replaced with

$$
\text { n.t. } \nabla \mathcal{S}^{+} 1_{\Delta^{*}}:=\lim _{\substack{Z \rightarrow x \\ Z \in \Gamma^{+}(x)}} \nabla \mathcal{S} 1_{\Delta^{*}}(Z) .
$$

By (4.2.18) and (4.2.19)

$$
\nu(x) 1_{\Delta^{*}}(x)=\lim _{\substack{Z \rightarrow x \\ Z \in \Gamma^{+}(x)}} \nabla \mathcal{S} 1_{\Delta^{*}}(Z)-\lim _{\substack{Z \rightarrow x \\ Z \in \Gamma^{-}(x)}} \nabla \mathcal{S} 1_{\Delta^{*}}(Z) .
$$

Thus, since $\Delta \subset \Delta^{*}$, by (4.3.14) and its analogue for $\mathcal{S}^{+}$, we obtain

$$
\left(f_{\Delta}\left|\nu(z)-f_{\Delta} \nu(y) d \sigma(y)\right|^{2} d \sigma(z)\right)^{\frac{1}{2}} \leq C \epsilon^{\gamma}
$$

for $x \in \partial \Omega \cap B_{0}$ and $0<r<M^{-1} r_{1}(\epsilon) \approx \epsilon^{1 /(8 n)} r_{1}(\epsilon)$. Hence, $\nu \in V M O_{l o c}(d \sigma)$. 
We now state a version of Theorem 4.3.1 when the pole for one or both of the Poisson kernels is infinity.

\section{Theorem 4.3.18.}

1. Suppose that $\Omega$ is an unbounded chord arc domain with $\Omega_{\text {ext }}$ connected, that $\log k_{1} \in V M O(d \sigma)$, where $k_{1}$ is the Poisson kernel for $\Omega$ with pole at infinity, and that $\log k_{2}^{X_{2}} \in V M O_{l o c}(d \sigma)$ where $k_{2}^{X_{2}}$ is the Poisson kernel for $\Omega_{\mathrm{ext}}$ with pole $X_{2} \in \Omega_{\text {ext }}$ then $\nu \in V M O_{l o c}(d \sigma)$.

2. Suppose $\Omega$ is an unbounded 2-sided chord-arc domain, with unbounded complement, and that $\log k_{1}, \log k_{2} \in V M O(d \sigma)$ where $k_{1}$ is the Poisson kernel for $\Omega$ with pole at infinity and $k_{2}$ is the Poisson kernel for $\Omega_{\mathrm{ext}}$ with pole at infinity then $\nu \in V M O_{l o c}(d \sigma)$.

As an immediate consequence to Theorem 4.3.1 and 4.3.18, we obtain the following, as in $[\mathrm{KT} 4]$.

Corollary 4.3.19. Suppose that $\Omega$ is a 2-sided chord-arc domain, and let $k_{1}, k_{2}$ be the Poisson kernels for $\Omega$ and for $\Omega_{\text {ext }}$, respectively, with fixed poles which may either be finite or infinite. Suppose also that $\log k_{1} \in C^{\alpha}$, and that $\log k_{2} \in V M O_{l o c}(d \sigma)$ (or $\log k_{2} \in V M O(d \sigma)$ if $k_{2}$ has pole at infinity). Then $\partial \Omega$ is locally the graph of a $C^{1, \alpha}$ function. In particular, if $\partial \Omega$ is bounded then $\Omega$ is a $C^{1, \alpha}$ domain.

Corollary 4.3.20. Suppose that $\Omega$ and $\Omega_{\text {ext }}$ and their respective Poisson kernels $k_{1}$ and $k_{2}$ satisfy the hypothesis of either Theorem 4.3 .1 or Theorem 4.3.18. Suppose also that $k_{1}$ satisfies the stronger condition that $\log k_{1} \in C_{l o c}^{\alpha}$ (or $C^{\alpha}$ if the pole of $k_{1}$ 
is infinite). Then $\partial \Omega$ is locally the graph of a $C^{1, \alpha}$ function. In particular, if $\partial \Omega$ is bounded then $\Omega$ is a $C^{1, \alpha}$ domain.

Let us indicate the proof the the Corollary in the case that $\partial \Omega$ is bounded. Observe that we may invoke Theorem 4.3.1 or Theorem 4.3.18 to deduce that $\nu \in V M O_{l o c}$. Consequently, by the aforementioned result of [HMT], we find that $\Omega$ is a vanishing Reifenberg domain. Thus, by the Alt-Caffarelli-Jerison theorem ([AC], $[\mathrm{J}]$ ), there exists $\rho>0$ such that for all $x \in \partial \Omega, \nu \in C^{\alpha}(B(x, \rho))$. If $\partial \Omega$ is unbounded, we first note that the results of $[\mathrm{AC}]$ and $[\mathrm{J}]$ are local in nature and then use a suitable local version of the [HMT] result.

Proof of Theorem 4.3.18. The proof is nearly the same as that of Theorem 4.3.1, with a few minor differences. Recall that in contrast to the situation for Theorem 4.3.1, we now impose the stronger assumption that $\Omega$ is a chord arc domain and that $\log k_{1} \in V M O$. In this setting we may use Lemma 4.2.22 and Lemma 4.2.25 as well as remove the dependence on $B_{0}$ from (4.2.1), Lemma 4.2.4 and Lemma 4.2.7. Following the proof of Theorem 4.3.1 we will be done if we show the following.

Fix $\epsilon \in(0,1)$, and let $r_{1} \in(0, \operatorname{diam} \Omega)$ be such that $(4.2 .1)$ holds with $\eta=\epsilon$, for $x \in \partial \Omega$ and $s \in\left(0, r_{1}\right)$. Without loss of generality we take $r_{1}<\min \left\{\frac{\delta\left(X_{1}\right)}{50}, 1\right\}$. Let $M:=50 \epsilon^{-\frac{1}{8 n}}$, set $r_{2}:=\frac{1}{M} r_{1}$, and suppose that $r \in\left(0, r_{2}\right)$. Given $x \in \partial \Omega$, let $x^{*}$ denote an arbitrary point in $\Gamma^{-}(x) \cap B(x, r / 2)$. Setting

$$
\Delta:=\Delta(x, r), \quad \Delta^{*}:=\Delta(x, M r)
$$

then it follows that

$$
\left(f_{\Delta}\left|\nabla \mathcal{S} 1_{\Delta^{*}}\left(z^{*}\right)-f_{\Delta} \nabla \mathcal{S} 1_{\Delta^{*}}\left(y^{*}\right) d \sigma(y)\right|^{2} d \sigma(z)\right)^{\frac{1}{2}} \leq C \epsilon^{\gamma},
$$


where $C=C(n, U R)$ and $\gamma$ is a positive constant, uniform in $x$ and $r$.

We again break up $1_{\Delta^{*}}$ as in (4.3.3) and estimate the left hand side of (4.3.21) by 3 terms, $I, I I$ and $I I I$. We estimate term $I$ the same way. We will show that

$$
I I=\left(f_{\Delta}\left|\nabla \mathcal{S}\left[\frac{k}{a}\right]\left(z^{*}\right)-f_{\Delta} \nabla \mathcal{S}\left[\frac{k}{a}\right]\left(y^{*}\right) d \sigma(y)\right|^{2} d \sigma(z)\right)^{\frac{1}{2}}=0
$$

for $k=k_{1}$ the Poisson kernel for $\Omega$ with pole at infinity, and for $z^{*}, y^{*}$ in $B(z, r / 2) \cap$ $\Gamma^{-}(z)$ and $B(y, r / 2) \cap \Gamma^{-}(y)$ respectively. Fix $x_{0} \in \partial \Omega$ and $R \gg r$, and let $\varphi_{R}(X) \in$ $C_{0}^{\infty}$ be a postivite smooth cutoff function on $B\left(x_{0}, R\right)$ such that $\operatorname{supp} \varphi \subset B\left(x_{0}, R\right)$, $|\nabla \varphi| \lesssim \frac{1}{R},\left|\nabla^{2} \varphi\right| \lesssim \frac{1}{R^{2}}$, and $\varphi \equiv 1$ on $B\left(x_{0}, \frac{R}{2}\right)$. Let

$$
\begin{aligned}
& I I_{R}:= \\
& \quad\left(f_{\Delta}\left|f_{\Delta} \int_{\partial \Omega}\left[\nabla \mathcal{E}\left(w-z^{*}\right)-\nabla \mathcal{E}\left(w-y^{*}\right)\right] \varphi_{R}(w)\left[\frac{k(w)}{a}\right] d \sigma(w) d \sigma(y)\right|^{2} d \sigma(z)\right)^{\frac{1}{2}} .
\end{aligned}
$$

If we set $A_{R}=B\left(x_{0}, R\right) \backslash B\left(x_{0}, \frac{R}{2}\right)$, let $g(X)$ be the Green function with pole at infinity, and let $\mathcal{L}:=\nabla \cdot \nabla$ denote the usual Laplacian in $\mathbb{R}^{n+1}$, then by definition

$$
\begin{aligned}
& I I_{R}^{2}= \\
& \begin{aligned}
& \frac{1}{a^{2}} f_{\Delta}\left|f_{\Delta} \iint_{\Omega} \mathcal{L}\left(\left[\nabla \mathcal{E}\left(W-z^{*}\right)-\nabla \mathcal{E}\left(W-y^{*}\right)\right] \varphi_{R}(W)\right) g(W) d W d \sigma(y)\right|^{2} d \sigma(z) \\
& \lesssim \frac{1}{a^{2}}\left(\iint_{A_{R}} g(W) \frac{1}{R^{n+2}} \frac{r}{R} d W\right)^{2} .
\end{aligned}
\end{aligned}
$$

Let $Y_{R}$ be a corkscrew point for $B\left(x_{0}, R\right)$ and $\Delta_{R}=\partial \Omega \cap B\left(x_{0}, R\right)$ then using Lemma 
4.2.25, Lemma 4.2.22 and (4.2.5) (without dependence on $B_{0}$ ) we have that

$$
\begin{aligned}
\frac{1}{a^{2}}\left(\iint_{A_{R}} g(W) \frac{1}{R^{n+2}} \frac{r}{R} d W\right)^{2} & \lesssim \frac{1}{a^{2}}\left(\frac{r}{R^{2}} \frac{1}{R^{n+1}} \iint_{B\left(x_{0}, R\right)} g(W) d W\right)^{2} \\
& \lesssim \frac{1}{a^{2}}\left(\frac{r}{R^{2}} g\left(Y_{R}\right)\right)^{2} \\
& \lesssim \frac{1}{a^{2}}\left(\frac{r}{R} \frac{\omega\left(\Delta_{R}\right)}{\sigma\left(\Delta_{R}\right)}\right)^{2} \\
& \lesssim\left(\frac{\sigma\left(\Delta\left(x_{0}, M r\right)\right)}{\sigma\left(\Delta_{R}\right)} \frac{\omega\left(\Delta_{R}\right)}{\omega\left(\Delta\left(x_{0}, M r\right)\right)}\right)^{2} \frac{r^{2}}{R^{2}} \\
& \lesssim \frac{r}{R},
\end{aligned}
$$

Thus, for fixed $z^{*}, y^{*}$ as above, $I I_{R} \rightarrow 0$ as $R \rightarrow \infty$, uniformly in the points $z^{*}$ and $y^{*}$ under consideration. By the Dominated Convergence Theorem, we see that

$$
I I=\lim _{R \rightarrow \infty} I I_{R}=0
$$

To handle term III we proceed as before until we have

$$
I I I \lesssim \sum_{j: 2^{j} \geq M}\left(f_{\Delta}\left[f_{\Delta} \int_{A_{j}^{\prime}} \frac{r}{\left(2^{j} r\right)^{n+1}} \frac{k(w)}{a} d \sigma(w) d \sigma(y)\right]^{2} d \sigma(z)\right)^{\frac{1}{2}} .
$$

This time we can estimate everything as we did for $I I I_{1}$ in the proof of Theorem 4.3.1 (since we have no dependence on $B_{0}^{\star}$ or $B_{0}$ ), this yields that

$$
\begin{aligned}
I I I \lesssim \sum_{k: 2^{k} \geq M} \frac{1}{2^{k}}\left(f_{\Delta}\left[f_{\Delta} f_{\Delta_{k}^{\prime}} \frac{k(w)}{a} d \sigma(w) d \sigma(y)\right]^{2} d \sigma(z)\right)^{\frac{1}{2}} \\
\leq \sum_{k: 2^{k} \geq M} \frac{1}{2^{k}} \frac{1}{a} \frac{\omega\left(\Delta_{k}^{\prime}\right)}{\sigma\left(\Delta_{k}^{\prime}\right)} \lesssim \sum_{k: 2^{k} \geq M} \frac{1}{2^{k}} \frac{\sigma(\Delta(x, M r))}{\sigma\left(\Delta_{k}^{\prime}\right)} \frac{\omega\left(\Delta_{k}^{\prime}\right)}{\omega(\Delta(x, M r))} \\
\lesssim \sum_{k: 2^{k} \geq M} \frac{1}{2^{k}}\left(\frac{M}{2^{k}}\right)^{n}\left(\frac{2^{k}}{M}\right)^{n+1 / 2} \lesssim \frac{1}{M} \lesssim \epsilon^{\frac{1}{8 n}}
\end{aligned}
$$

which proves the theorem. 


\section{Bibliography}

[AH] D. Adams and L. Hedberg, Function spaces and potential theory, volume 314 of Grundlehren der Mathematischen Wissenschaften [Fundamental Principles of Mathematical Sciences] (corrected second printing). Springer-Verlag, Berlin, 1999.

[ABaHM] M. Akman, M. Badger, S. Hofmann and J.M. Martell, Rectifiability and elliptic measures on 1-sided NTA domains with Ahlfors-David regular boundaries. Preprint 2015. arXiv:1507.02039

[AC] H. W. Alt and L. A. Caffarelli, Existence and regularity for a minimum problem with free boundary, J. Reine Agnew. Math. 325 (1981) 105-144

[AHLT] P. Auscher, S. Hofmann, J.L. Lewis and P. Tchamitchian, Extrapolation of Carleson measures and the analyticity of Kato's square-root operators, Acta Math. 187 (2001), no. 2, 161-190.

[AHM ${ }^{3}$ TV] J. Azzam, S. Hofmann, J.M. Martell, S. Mayboroda, M. Mourgoglou, X. Tolsa, A. Volberg, Rectifiability of harmonic measure. Preprint 2015. arXiv:1509.06294.

[AHMNT] J. Azzam, S. Hofmann, J. M. Martell, K. Nyström, T. Toro, A new characterization of chord-arc domains, preprint 2014. arXiv:1406.2743

[AHMTT] P. Auscher, S. Hofmann, C. Muscalu, T. Tao and C. Thiele, Carleson measures, trees, extrapolation, and $T(b)$ theorems, Publ. Mat. 46 (2002), no. $2,257-325$.

[Azz] J. Azzam. Tangents, rectifiability, and corkscrew domains. Preprint 2015. arXiv:1505.03960.

[AS] J.Azzam and R. Schul, Hard Sard: quantitative implicit function and extension theorem for Lipschitz maps, Geom. Funct. Anal. 22 (2012), 1062-1123

[Ba] M. Badger. Null sets of harmonic measure on NTA domains: Lipschitz approximation revisited. Math. Z., 270(1-2):241-262, 2012.

[BL] B. Bennewitz and J.L. Lewis, On weak reverse Hölder inequalities for nondoubling harmonic measures, Complex Var. Theory Appl. 49 (2004), 571-582. 
[BiJ] C. Bishop and P. Jones, Harmonic measure and arclength, Ann. of Math. (2) 132 (1990), 511-547.

[Bo] J. Bourgain, On the Hausdorff dimension of harmonic measure in higher dimensions, Invent. Math. 87 (1987), 477483.

[CFMS] L. Caffarelli, E. Fabes, S. Mortola and S. Salsa, Boundary behavior of nonnegative solutions of elliptic operators in divergence form. Indiana Univ. Math. J. 30 (1981), no. 4, 621-640.

[Car] L. Carleson, Interpolation by bounded analytic functions and the corona problem, Ann. of Math. (2) 76 (1962), 547-559.

[CG] L. Carleson and J. Garnett, Interpolating sequences and separation properties, J. Analyse Math. 28 (1975), 273-299.

[Chr] M. Christ, A $T(b)$ theorem with remarks on analytic capacity and the Cauchy integral, Colloq. Math., LX/LXI (1990), 601-628.

[Da] B. Dahlberg, On estimates for harmonic measure, Arch. Rat. Mech. Analysis 65 (1977), 272-288.

[DJ] G. David and D. Jerison, Lipschitz approximation to hypersurfaces, harmonic measure, and singular integrals, Indiana Univ. Math. J. 39 (1990), no. $3,831-845$.

[DS1] G. David and S. Semmes, Singular integrals and rectifiable sets in $\mathbb{R}^{n}$ : Beyond Lipschitz graphs, Asterisque 193 (1991).

[DS2] G. David and S. Semmes, Analysis of and on Uniformly Rectifiable Sets, Mathematical Monographs and Surveys 38, AMS 1993.

[DS3] G. David and S. Semmes, Quantitative Rectifiability and Lipschitz Mappings, Trans. Amer. Math. Soc. 337 (1993), no. 2, 855-889

[EG] L.C. Evans and R.F. Gariepy, Measure Theory and Fine Properties of Functions, Studies in Advanced Mathematics, CRC Press, Boca Raton, FL, 1992.

[GR] J. García-Cuerva, J. L.Rubio de Francia Weighted norm inequalities and related topics, North-Holland Mathematics Studies, 116. North-Holland Publishing Co., Amsterdam, 1985.

[GM] J.B. Garnett and D.E. Marshall. Harmonic measure. New Mathematical Monographs, 2. Cambridge University Press, Cambridge, 2005.

[HKM] J. Heinonen, T. Kilpeläinen, and O. Martio. Nonlinear Potential Theory of Degenerate Elliptic Equations. Dover Publications Inc., 2006.

[HL] S. Hofmann and J.L. Lewis, The Dirichlet problem for parabolic operators with singular drift terms, Mem. Amer. Math. Soc. 151 (2001), no. 719. 
[HM1] S. Hofmann and J.M. Martell, $A_{\infty}$ estimates via extrapolation of Carleson measures and applications to divergence form elliptic operators, Trans. Amer. Math. Soc. 364 (2012), no. 1, 65-101

[HM2] S. Hofmann and J.M. Martell, Uniform rectifiability and harmonic measure I: Uniform rectifiability implies Poisson kernels in $L^{p}$, Ann. Sci. École Norm. Sup., to appear.

[HM3] S. Hofmann and J.M. Martell, Uniform Rectifiability and harmonic measure IV: Ahlfors regularity plus Poisson kernels in $L^{p}$ implies uniform rectifiability. Preprint 2015. arXiv:1505.06499.

[HMM] S. Hofmann, J.M. Martell, and S. Mayboroda, Uniform rectifiability, Carleson measure estimates and approximation of harmonic functions, preprint

[HMU] S. Hofmann, J.M. Martell and I. Uriarte-Tuero, Uniform rectifiability and harmonic measure II: Poisson kernels in $L^{p}$ imply uniform rectifiability, Duke Math. J. 163 (2014), no. 8, 1601-1654.

[HMMM] S. Hofmann, D. Mitrea, M. Mitrea, A. Morris, $L^{p}$-Square Function Estimates on Spaces of Homogeneous Type and on Uniformly Rectifiable Sets, preprint 2103. arXiv:1301.4943

[HMT] S. Hofmann, M. Mitrea and M. Taylor, Singular integrals and elliptic boundary problems on regular Semmes-Kenig-Toro domains, Int. Math. Res. Not. 2010, no. 14 2567-2865

$[\mathrm{J}] \quad$ D. Jerison, Regularity of the Poisson kernel and free boundary problems, Colloq. Math. 60-61 (1990), 547-567

[JK] D. Jerison and C. Kenig, Boundary behavior of harmonic functions in nontangentially accessible domains, Adv. in Math. 46 (1982), no. 1, 80147.

[Kel] O. Kellog, Foundations of Potential Theory, Springer, Berlin, 1929 (reprinted by Dover, N.Y., 1953).

[KT1] C. Kenig and T. Toro, Harmonic measure on locally flat domains, Duke Math. J. 87, (1997), 509-551

[KT2] C. Kenig and T. Toro, Free Boundary Regularity for harmonic measures and Poisson kernels, Ann. Math. 150 (1999), 369-454

[KT3] C. Kenig and T. Toro, Poisson kernel characterization of Reifenberg flat chord arc domains, Ann. Sci. Ecole Norm. Sup. (4) 36 (2003), no.3, 323401

[KT4] C. Kenig and T. Toro, Free boundary regularity below the continuous threshold: 2 phase problems, J. Reine Angew Math. 596 (2006), 1-44 
[KP] O. Kowalski and D. Preiss, Besicovitch-type properties of measures and submanifolds, J. Reine Angew Math. 379 (1987), 115-151.

[La] M. Lavrentiev, Boundary problems in the theory of univalent functions (Russian), Math Sb. 43 (1936), 815-846; AMS Transl. Series 232 (1963), $1-35$.

[LM] J. Lewis and M. Murray, The method of layer potentials for the heat equation in time-varying domains, Mem. Amer. Math. Soc. 114 (1995), no. 545 .

[LN] J. L. Lewis and K. Nyström, Regularity and free boundary regularity for the $p$-Laplace operator in Reifenberg flat and Ahlfors regular domains, Journal Amer. Math. Soc. 25 (2012), 827-862.

[M] P. Mattila, Geometry of sets and measures in Euclidean spaces. Fractals and rectifiability, Cambridge Studies in Advanced Mathematics 44, Cambridge University Press, Cambridge, 1995.

[MMV] P. Mattila, M. Melnikov and J. Verdera, The Cauchy integral, analytic capacity, and uniform rectifiability, Ann. of Math. (2) 144 (1996), no. 1, $127-136$.

[Maz] V. Maz'ya. Sobolev spaces. Springer Series in Soviet Mathematics. Springer-Verlag, Berlin, 1985. Translated from the Russian by T. O. Shaposhnikova.

[Mo] M. Mourgoglou, Uniform domains with rectifiable boundaries and harmonic measure. Preprint 2015. arXiv:1505.06167

$[\mathrm{NToV}] \quad$ F. Nazarov, X. Tolsa, and A. Volberg, On the uniform rectifiability of ad-regular measures with bounded Riesz transform operator: The case of codimension 1, Acta Math., to appear.

[RR] F. and M. Riesz, Über die randwerte einer analtischen funktion, Compte Rendues du Quatrième Congrès des Mathématiciens Scandinaves, Stockholm 1916, Almqvists and Wilksels, Upsala, 1920.

[Se] S. Semmes, Analysis vs. geometry on a class of rectifiable hypersurfaces in $\mathbb{R}^{n}$, Indiana Univ. Math. J. 39 (1990), 1005-1035.

[Ste] E. M. Stein, Singular Integrals and Differentiability Properties of Functions, Princteon University Press, Princeton, NJ, 1970.

[W] J. Wu. On singularity of harmonic measure in space. Pacific J. Math., 121(2):485-496, 1986.

[Z] W. Ziemer. Some remarks on harmonic measure in space. Pacific J. Math., 55:629-637, 1974. 


\section{VITA}

Simon Bortz was born to Mark and Liza Bortz on March 30, 1987 in Highland Park, IL. Mark played professional football for the Chicago Bears and Liza had a degree in X-ray technology. He grew up in Libertyville, IL where he attended Libertyville High School. After a brief time at Southern Illinois University - Edwardsville he transferred to Indiana University where he completed his BS in Mathematics. In May 2014 he

was married to his wife Tiffanie Bortz. Simon spent two years in industry before entering into graduate school at the University of Missouri to study Mathematics. His mathematical interests are primarily in the field of analysis and include partial differential equations, geometric measure theory and harmonic analysis. 